SLAC-R-768

First Observation of the Parity Violating Asymmetry in Moller Scattering

Imran Younus

Stanford Linear Accelerator Center, Stanford University, Stanford, CA 94309

Work supported by Department of Energy contract DE-AC02-76SF00515. 


\begin{abstract}
This thesis reports on the E158 experiment at Stanford Linear Accelerator Center (SLAC), which has made the first observation of the parity non-conserving asymmetry in Moller scattering. Longitudinally polarized $48 \mathrm{GeV}$ electrons are scattered off unpolarized (atomic) electrons in a liquid hydrogen target with an average $Q^{2}$ of $0.027 \mathrm{GeV}^{2}$. The asymmetry in this process is proportional to $\left(\frac{1}{4}-\sin ^{2} \theta_{W}\right)$, where $\sin ^{2} \theta_{W}$ gives the weak mixing angle.

The thesis describes the experiment in detail, with a particular focus on the design and construction of the electromagnetic calorimeter. This calorimeter was the primary detector in the experiment used to measure the flux of the scattered Moller electrons and $e P$ electrons. It employed the quartz fiber calorimetry technique, and was built at Syracuse University.

The preliminary results from the first experimental data taken in spring 2002 give $A_{P V}=-151.9 \pm 29.0($ stat $) \pm 32.5$ (syst) parts per billion. This in turn gives $\sin ^{2} \theta_{W}=0.2371 \pm 0.0025 \pm 0.0027$, which is consistent with the Standard Model prediction $(0.2386 \pm 0.0006)$.
\end{abstract}




\title{
FIRST OBSERVATION OF THE PARITY VIOLAING ASYMMETRY IN MOLLER SCATTERING
}

\section{IMRAN YOUNUS}

\author{
DISSERTATION \\ Submitted in partial fulfillment of the requirements for the \\ degree of Doctor of Philosophy in Physics \\ in the Graduate School of Syracuse University
}

November 2003

Approved $\ldots \ldots \ldots \ldots \ldots \ldots \ldots \ldots \ldots$

Professor Paul Souder

Date 
(c) Copyright 2003 Imran Younus

All rights reserved 


\section{Contents}

1 Parity Violating Asymmetries in Polarized Electron Scattering 1

1.1 Introduction . . . . . . . . . . . . . . . . . . 1

1.2 Impact of Precision Electroweak Measurement . . . . . . . . . . . . 3

1.3 Parity Violating Electron Scattering . . . . . . . . . . . . . . 6

1.3.1 $A_{P V}$ Away From $Z$ Pole . . . . . . . . . . . . . . . . . 7

1.4 Left-Right Asymmetry in Moller Scattering . . . . . . . . . . . . 8

1.5 Radiative Corrections and Running of $\sin ^{2} \theta_{W}$. . . . . . . . . . 11

1.6 "New Physics" Sensitivity . . . . . . . . . . . . . . . . 14

1.6.1 $Z^{\prime}$ Bosons ....................... 15

1.6.2 Other Contact Interactions . . . . . . . . . . . . . . 16

1.6.3 Oblique Corrections . . . . . . . . . . . . 17

2 Experimental Design $\quad 19$

2.1 Experimental Considerations . . . . . . . . . . . . . . . . 19

2.1.1 Figure of Merit . . . . . . . . . . . . . 20

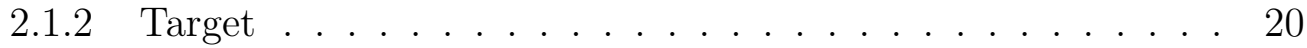

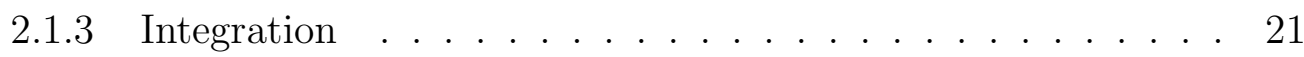

2.1.4 Spectrometer ..................... 21 
2.1.5 Beam Monitoring ................ 23

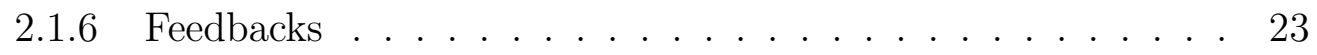

2.2 Physics Backgrounds . . . . . . . . . . . . . . . . . . . 23

2.2.1 Electron Background . . . . . . . . . . . . . . . 24

2.2 .2 Pions . . . . . . . . . . . . . . . . 25

2.2.3 Synchrotron Radiation .............. . 25

2.2.4 Neutral Background . . . . . . . . . . . . . . 26

2.3 Estimate of Rate . . . . . . . . . . . . . . . . . . . . 27

2.4 Polarized Electron Source . . . . . . . . . . . . . . . . . . . 28

2.4.1 Circular Polarization . . . . . . . . . . . . . . . . . 29

2.4.2 Insertable Half Wave Plates . . . . . . . . . . . . . . . . . . 30

2.4 .3 Asymmetry Inverter . . . . . . . . . . . . . . . . . 31

2.4.4 Cathode .................... . . 32

2.4.5 Helicity Sequence . . . . . . . . . . . . . . . 32

2.5 Accelerator . . . . . . . . . . . . . . . . . . . 33

2.5.1 A-Line . . . . . . . . . . . . . . . . 34

2.5.2 Beam Rate and Spills . . . . . . . . . . . . . . . . . 35

2.6 Beam Monitoring . . . . . . . . . . . . . 36

2.6.1 Beam Current Monitors . . . . . . . . . . . . 36

2.6.2 Beam Position Monitors . . . . . . . . . . . . . 37

2.6.3 Synchrotron Light Monitor . . . . . . . . . . . . . . . . . . . 39

2.6.4 Wire Array . . . . . . . . . . . . . . . . . 40

2.6.5 Beam Dithering Hardware . . . . . . . . . . . . . . . . . . . 41

2.7 Liquid Hydrogen Target . . . . . . . . . . . . . . . . . . . 41

2.7.1 Moller Polarimeter and Carbon Targets . . . . . . . . . . . . 44

2.8 Spectrometer and Collimators . . . . . . . . . . . . . . . . 44 
2.8 .1 Dipole Chicane . . . . . . . . . . . . . . . . . . 46

2.8.2 Photon Collimator . . . . . . . . . . . . . . . . 48

2.8.3 Momentum Collimator . . . . . . . . . . . . . . . . . . . . 49

2.8.4 Synchrotron Collimators . . . . . . . . . . . . . 50

2.8 .5 "Holey" Collimator . . . . . . . . . . . . . . . . 51

2.8 .6 Quadrupole Magnets . . . . . . . . . . . . . . . . . 52

2.8 .7 Drift Pipe . . . . . . . . . . . . . . . 53

2.8 .8 Collimator Masks . . . . . . . . . . . . . . . . . . 53

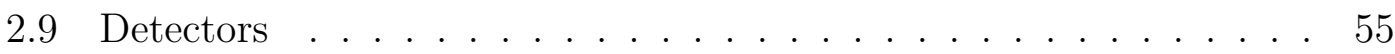

2.9 .1 Profile Detector . . . . . . . . . . . . . . 55

2.9.2 Pion Detector . . . . . . . . . . . . . . . . 57

2.9 .3 Polarimeter . . . . . . . . . . . . . . . . . . 58

2.9 .4 Luminosity Monitor . . . . . . . . . . . . . . . . . 59

2.10 Data Aquisition System _ . . . . . . . . . . . . . . 61

2.10 .1 Integrating ADCs . . . . . . . . . . . . . . 61

2.11 Helicity Correlated Feedbacks _. . . . . . . . . . . . . 63

2.12 Online Monitoring . . . . . . . . . . . . . . . . 65

$\begin{array}{lll}3 & \text { E158 Calorimeter } & 67\end{array}$

3.1 Moller Detector . . . . . . . . . . . . . . . . 67

3.1 .1 Detector Geometry . . . . . . . . . . . . . . 70

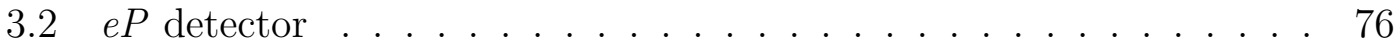

3.3 Putting It All Together . . . . . . . . . . . . . . . 77

3.3 .1 Cleaving . . . . . . . . . . . . . . . . 78

3.3 .2 Constructing Layers . . . . . . . . . . . . . . . . . 80

3.3 .3 Bundling the Fibers $\ldots \ldots \ldots$. . . . . . . . . 83 
3.3.4 Mirrors, Light Guides, and PMT Assemblies . . . . . . . . . 85

3.3.5 Lead Shielding . . . . . . . . . . . . . . 87

3.4 Linearity of the Photomultiplier Tubes . . . . . . . . . . . . 88

3.5 Moller Detector Electronics . . . . . . . . . . . . . . . . . . . 92

4 Preliminary Results for $A_{P V}$ and $\sin ^{2} \theta_{W} \quad 97$

4.1 Data Processing . . . . . . . . . . . . . . . . 97

4.1.1 Pedestal Subtraction . . . . . . . . . . . . . . . . . . 98

4.1 .2 Data Cuts . . . . . . . . . . . . . . 98

4.2 Measuring an Asymmetry . . . . . . . . . . . . . . . . . . . . . . . 99

4.3 Moller Detector Asymmetry . . . . . . . . . . . . . . 103

4.4 eP Asymmetry . . . . . . . . . . . . . . . . 105

4.5 Physics Asymmetry . . . . . . . . . . . . . . . 106

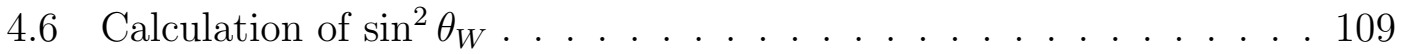

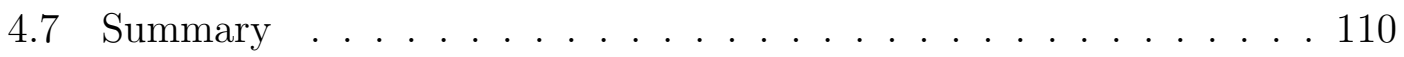

A First Results from E158 113 


\section{List of Figures}

1.1 Neutral current amplitudes leading to the asymmetry $A_{L R}$ at the tree level. . . . . . . . . . . . . . . . . . . 8

$1.2 \gamma Z$ mixing diagrams, and $W$-loop contribution to anapole moment. 12

1.3 Box diagrams with two heavy bosons. . . . . . . . . . . . . . 13

1.4 Boxes containing one photon and $Z$-loop contribution to the anapole moment. . . . . . . . . . . . . . . . 13

1.5 Predicted running of $\sin ^{2} \theta_{W}\left(Q^{2}\right)$ from the precision measurement at the $Z^{\circ}$ resonance. . . . . . . . . . . . . . . . . 15

2.1 The behavior of the asymmetry, the differential cross section and the figure of merit as a function of $\left|\cos \theta_{c m}\right| \ldots \ldots 20$

2.2 An overview of the Polarized Electron Source as it is configured for

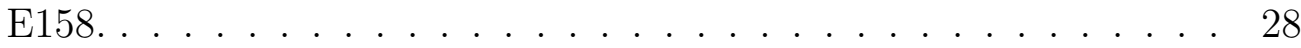

2.3 The Helicity Control Bench containing the optics for control of the laser beam polarization and beam asymmetries. . . . . . . . . . . 30

2.4 Beam Diagnostics for E158. . . . . . . . . . . . . . . 36

2.5 Resolution of different beam monitors. . . . . . . . . . . . . . . . 39

2.6 Schematic for the synchrotron light monitor. . . . . . . . . . . . . . 40 
2.7 Typical output from the wire array, averaged over a one second period. 41

2.8 A schematic of the liquid hydrogen target loop. . . . . . . . . . . . 42

2.9 Examples of the wire mesh disks. . . . . . . . . . . . . . . 43

2.10 Overview of the experimental setup in End Station A. . . . . . . . . 45

2.11 A top view schematic of the layout of the E158 spectrometer. . . . . 45

2.12 Cad diagram of the assembly "3DC2C" between the first two dipoles that contains the first photon collimator. . . . . . . . . . . . 48

2.13 Momentum collimator, and the Moller and $e P$ scattered flux at the acceptance defining collimator. . . . . . . . . . . . . . . . 49

2.14 A photograph of the momentum collimator and the holey collimator in their segment of beam pipe. . . . . . . . . . . . . . 51

2.15 Measured electron profile at the detector plane with quadrupole magnets off and on. . . . . . . . . . . . . . . . . . . 52

2.16 Schematic of the drift pipe, showing relative positions of the collimator masks as well as the synchrotron collimators. . . . . . . . . 54

2.17 Schematic of the E158 detector package. . . . . . . . . . . . 55

2.18 Overhead view of the Moller detector ("Calorimeter"), the pion detector, the profile detector ("Annulus" with "Cerenkov scanners"), the polarimeter. . . . . . . . . . . . . . 56

2.19 Schematic of the Profile detector wheel, showing four Cerenkov counters sitting on their movable drives, and Single Cerenkov counter assembly. . . . . . . . . . . . . . . . . 57

2.20 A diagram of the polarimeter detector . . . . . . . . . 58

2.21 Schematic of the front view of the luminosity monitor. . . . . . . . 60

2.22 Schematic of DAQ system. . . . . . . . . . . . . . . . 62

2.23 Different screen shots of the online monitoring software. . . . . . . . 66 
3.1 Fibers used for the Moller detector. . . . . . . . . . . . . . . . . . 69

3.2 Moller detector outline . . . . . . . . . . . . . . . . 70

3.3 Moller and $e P$ detector cad diagram . . . . . . . . . . . . . . 71

3.4 The shape of the $\mathrm{Cu}$ plate for the Moller detector. . . . . . . . . . . 72

3.5 Moller $\mathrm{Cu}$ plate and a layer of fibers . . . . . . . . . . . . . . 73

3.6 Radii of active regions of Moller and $e P$ detectors. . . . . . . . . . . 73

3.7 Back plate of the detector assembly showing the cookies where the fibers are collected, and the Mirrors, light guides and cylinders that hold the PMTs. . . . . . . . . . . . . . . . . . 74

3.8 Complete Moller and eP detectors. . . . . . . . . . . . . . . . . 75

3.9 The shape of a $\mathrm{Cu}$ plate for the $e P$ detector. . . . . . . . . . . 76

3.10 eP $\mathrm{Cu}$ plate and a layer of fibers. . . . . . . . . . . . . . 77

3.11 Schematic of the cutter used to cut the Moller fibers. . . . . . . . . 78

3.12 Cleaved ends of Moller fibers. . . . . . . . . . . . . . . . 78

3.13 Cleaved ends of $e P$ fibers. . . . . . . . . . . . . . . . 79

3.14 Glued ends of Moller fibers. . . . . . . . . . . . . . . . . . . 80

3.15 The jig used to produce layers of fibers for Moller detector. . . . . . 81

3.16 The complete process of creating layers of Moller fibers. . . . . . . . 82

3.17 Empty spaces in the channel in a Moller $\mathrm{Cu}$ plate. . . . . . . . . . . 82

3.18 The jig used to produce layers of fibers for $e P$ detector. . . . . . . . 83

3.19 cookies and cookie plate. . . . . . . . . . . . . . . . 84

3.20 Inner, middle and outer, and eP cookies; Elegant view of the back of the detector showing the cookies and cookie plates! . . . . . . . 85

3.21 Mirrors, light guide and PMT assembly. . . . . . . . . . . . . . . . 86

3.22 Mirrors and air light guides. . . . . . . . . . . . . . . . 86

3.23 Schematic diagram of the lead shielding. . . . . . . . . . . . . 87 
3.24 Complete E158 detector with the lead shielding. . . . . . . . . . . . 87

3.25 Dynode voltage distribution ratios. . . . . . . . . . . . . 88

3.26 Schematic of the test setup. . . . . . . . . . . . . . 89

3.27 Typical set of linearity plots produced for all PMTs in the benchtest. 91

3.28 Linearity of PMTs at expected light levels vs PMT\#. . . . . . . . . 92

3.29 Diagram of the Moller detector electronics layout. . . . . . . . . . . 93

3.30 A plot of $\sigma_{\text {Moller }}^{2}$ vs $1 /$ (beam intensity) for different beam intensities. 94

4.1 Moller asymmetry distribution for one run, starting from the leftright asymmetry in the detected signal from one PMT to average asymmetry of complete Moller detector. . . . . . . . . . . . . 102

4.2 Moller detector asymmetry vs slug number. . . . . . . . . . . . . 103

4.3 Moller detector asymmetry pull plots. . . . . . . . . . . . . . . . . . 104

4.4 Moller detector asymmetry for all slugs, and the average asymmetry for each energy and half wave plate state. . . . . . . . . . . . . . . 104

4.5 eP detector asymmetry, the sign of the asymmetry is not corrected for the half wave plate state and energy state. . . . . . . . . . . 105

4.6 Comparison between the profile detector data and Monte Carlo simulation, with and without holey collimator. . . . . . . . . 107

4.7 Comparison of E158 results with the standard model prediction and other experiments . . . . . . . . . . . . . . . . 111 


\section{List of Tables}

2.1 Parameters of the Flash:Ti laser beam (for E158 2002 Physics Run I). 29

4.1 The dilutions and corrections to the Moller asymmetry from the elastic and inelastic $e P$ backgrounds. . . . . . . . . . . 106

4.2 Summary of the corrections to the Moller detector asymmetries. . . 108

4.3 Summary of the dilution factors for the Moller detector asymmetries.109 


\section{Chapter 1}

\section{Parity Violating Asymmetries in Polarized Electron Scattering}

\subsection{Introduction}

In 1956, after reviewing the experimental data then available, Lee and Yang asserted that the weak interactions did not conserve parity [1], i.e., they were not invariant under spatial inversion. One year later, $\mathrm{Wu}$ and collaborators verified the parity violating nature of the weak interactions in beta decay of ${ }^{60} \mathrm{Co}[2]$. Further studies have demonstrated the vector-axial vector structure of weak interactions and showed that it is maximally parity violating.

In the late 1960s, Salam, Weinberg and Glashow showed that the electromagnetic and weak interactions can be treated as different aspects of a single electroweak interaction. They predicted that this symmetry between electromagnetic and weak interactions would be evident at very large momentum transfer $\left(q^{2} \gg 10^{4} \mathrm{GeV}^{2}\right)$. But at low energies, it would be a broken symmetry: of the 
four mediating vector bosons involved, one (the photon) would be massless and the others, $W^{+}, W^{-}, Z^{\circ}$, would be massive. The theory contains an arbitrary constant, the weak mixing angle denoted by $\sin ^{2} \theta_{W}$, to be determined by experiments. Over the last three decades, this model has been verified experimentally with everincreasing accuracy, and has come to be known as the "Standard Model".

Although neutrino experiments proved the existence of neutral weak currents in 1973, independent confirmation was important and was provided in 1978 by the SLAC E122 experiment, which detected parity violation effects due to $Z^{\circ}$ and $\gamma$ exchange in the inelastic scattering of polarized electrons by deuterons [3]. The weak mixing angle $\theta_{W}$ can be extracted from parity violating asymmetry, and the value obtained in E122 experiment was $\sin ^{2} \theta_{W}=0.224 \pm 0.020$ [3], which was consistent with the standard model predictions.

The results from E122 were crucial in asserting the Salam-Weinberg-Glashow theory to be the "correct" model describing electroweak interaction. E122's measurement of an asymmetry of order $\sim 10^{-4}$ pioneered techniques of measuring extremely small parity violating asymmetries.

Over the past decade, experiments studying weak interactions at the $Z^{\circ}$ resonance have measured weak neutral current observables, such as $\sin ^{2} \theta_{W}$, with spectacular precision. On the other hand, at low $Q^{2}$, tests of the electroweak theory in the weak neutral current sector are typically less sensitive by more than an order of magnitude.

SLAC E158 $[4,5]$ experiment plans to test the standard model at low momentum transfer with sensitivity to potential new physics. This experiment will provide a precise measurement of parity violating asymmetry in the scattering of a longitudinally polarized electron beam off the atomic electrons in a liquid hydrogen target, at $Q^{2} \approx 0.025 \mathrm{GeV}^{2}$. E158 plans to measure $\sin ^{2} \theta_{W}$ to $\sim 0.0008$, which 
would establish the $Q^{2}$ dependence of the weak coupling angle at a significance of $\sim 8 \sigma$ within the context of Standard Model.

\subsection{Impact of Precision Electroweak Measure- ment}

There are several ways to look for physics beyond standard model. One is to study interactions at very high energies in high energy colliders. Secondly, we can look for rare or forbidden processes or search for any violations in the symmetries of the model. Alternatively, we can probe the electroweak one loop structure. All these approaches are complementary to each other. The following section, adapted from [6-8], outlines the status of several precisely measured electroweak parameters, and the natural relations among them and the radiative corrections.

The $\mathrm{SU}(2)_{L} \times \mathrm{U}(1)_{Y}$ electroweak sector of the standard model contains 17 or more fundamental parameters. They include gauge and Higgs field couplings as well as fermion masses and mixing angles. In terms of these parameters, predictions can be made with high accuracy for essentially any electroweak observable. Very high precision measurements of these quantities can then be used to test the standard model, even at quantum loop level, or search for small deviations from expectations which would indicate "new physics".

Some fundamental electroweak parameters have been determined with extraordinary precision. Foremost in that category is the fine structure constant $\alpha$. It is best obtained by comparing the measured anomalous magnetic moment of the 
electron [9], $a_{e} \equiv\left(g_{e}-2\right) / 2$, with the 4 loop QED prediction

$$
\alpha^{-1}=137.03599959(40)
$$

After $a_{e}$, the next best (direct) measurement of $\alpha$ comes from the quantum hall effect

$$
\alpha^{-1}=137.03600370(270)
$$

which is not nearly as precise. Nevertheless the agreement between 1.2.1 and 1.2.2 tests QED up to 4 loop level.

The usual fine structure constant is defined at zero momentum transfer which is not well suited for short-distance electroweak effects. Vacuum polarization loops screen charges such that the effective electric charge increases at short-distances. One can incorporate those quantum loop contributions into a short-distance $\alpha\left(m_{Z}\right)$ [10] defined at $q^{2}=m_{Z}^{2}$. The main effect comes from lepton loops which can be very precisely calculated and somewhat smaller hadronic loops. A recent study finds $[11]$

$$
\alpha^{-1}\left(m_{Z}\right)=128.933(21)
$$

In weak interaction physics, the most precisely determined parameter is the Fermi constant $G_{F}$, which is extracted by comparing the experimental value of muon lifetime with the theoretical prediction

$$
G_{F}=1.16637(1) \times 10^{-5} \mathrm{GeV}^{-2}
$$


Gauge boson masses are not as well determined as $G_{F}$, but they have reached high level of precision. In particular, the $Z$ mass has been measured with high statistics Breit-Wigner fits to the $Z$ resonance at LEP with the result

$$
m_{Z}=91.1867(21) \mathrm{GeV} \text {. }
$$

In case of $W^{ \pm}$bosons, the mass is obtained from studies at $p \bar{p}$ colliders

$$
m_{W}=80.39(6) \mathrm{GeV} \text {. }
$$

The current level of uncertainty, $\pm 60 \mathrm{MeV}$ is large compared to $\Delta m_{Z}$.

In addition to masses, the renormalized weak mixing angle plays a central role in tests of the standard model. Currently, $Z$ pole studies at LEP and SLAC give

$$
\sin ^{2} \theta_{W}\left(m_{Z}\right)_{\overline{M S}}=0.23100 \pm 0.00022
$$

where $\sin ^{2} \theta_{W}\left(m_{Z}\right)_{\overline{M S}}$ is defined in modified minimal subtraction scheme. Numerically, this is related to $\sin ^{2} \theta_{W}^{e f f}$ as [8]

$$
\sin ^{2} \theta_{W}^{e f f}=\sin ^{2} \theta_{W}\left(m_{Z}\right) \overline{M S}+0.00028
$$

All of the above precision measurements can be collectively used to test the standard model, predict the Higgs mass and search for "new physics". The ability stems from the natural relations in the quantities and calculations of radiative corrections to them [8]: 


$$
\begin{aligned}
\frac{\pi \alpha}{\sqrt{2} G_{F} m_{W}^{2}} & =\left(1-\frac{m_{W}^{2}}{m_{Z}^{2}}\right)(1-\Delta r), \\
& =\sin ^{2} \theta_{W}\left(m_{Z}\right)_{\overline{M S}}\left(1-\Delta r\left(m_{Z}\right) \overline{M S}\right) \\
\frac{4 \pi \alpha}{\sqrt{2} G_{F} m_{Z}^{2}} & =\sin ^{2} 2 \theta_{W}\left(m_{Z}\right)_{\overline{M S}}(1-\Delta \hat{r}) .
\end{aligned}
$$

The expressions for $\Delta r, \Delta r\left(m_{Z}\right)_{\overline{M S}}$ and $\Delta \hat{r}$ contains all one loop corrections to $\alpha$, muon decay, $m_{W}, m_{Z}$, and $\sin ^{2} \theta_{W}\left(m_{Z}\right) \overline{M S}$ and incorporate some leading two loop contributions. The quantities $\Delta r$ and $\Delta \hat{r}$ are particularly interesting because of their dependence on $m_{t}$ and $m_{H}$. In addition, all three quantities provide probes of "new physics".

Such studies and the comparison between different measurements have tested the standard model at the $0.1 \%$ level. As a byproduct, they have been used to predict the large top quark mass and now suggest a relatively light Higgs mass. The good agreement between theory and experiment severly constrains the possible "new physics" one can append to the Standard Model.

\subsection{Parity Violating Electron Scattering}

The scattering of longitudinally polarized (left or right-handed) electrons on an unpolarized target provides a clean window to study weak neutral current interactions. These experiments measure the left-right scattering asymmetry defined by

$$
A_{P V} \equiv \frac{\sigma_{R}-\sigma_{L}}{\sigma_{R}+\sigma_{L}}
$$


where $\sigma_{R}\left(\sigma_{L}\right)$ is the scattering cross section using incident right(left) handed electrons. $A_{P V}$ is manifestly parity violating and measures the interference between electromagnetic and weak neutral current amplitudes. A classic example is the famous SLAC asymmetry measurement for deep-inelastic polarized $e-D$ scatter$\operatorname{ing}[3]$.

For $Q^{2} \ll M_{Z}^{2}, A_{P V}$ is proportional to the ratio of weak and electromagnetic amplitudes, and rises with $Q^{2}[12]$. At $Q^{2} \sim 1 \mathrm{GeV}^{2}, A_{P V} \approx 10^{-4}$. The SLAC experiment mentioned above was the first to device techniques required to measure such small asymmetries. Since that pioneering effort, the statistical and systematic precision achievable in the raw asymmetry measurement in low $Q^{2}$ polarized electron scattering has improved steadily. A later measurement of elastic polarized $e-C$ scattering [13] has achieved a statistical precision of $1.4 \times 10^{-7}$ and a systematic error of $2 \times 10^{-8}$.

\subsection{1 $A_{P V}$ Away From $Z$ Pole}

One of the most precise measurements of $A_{P V}$ comes from SLD experiment. The value is obtained for $Q^{2} \sim m_{Z}^{2}$ where the amplitude for $Z$ exchange, $A_{Z}$, becomes imaginary. If there is some other interaction present with amplitude

$$
\begin{aligned}
A_{X} & \propto \frac{1}{Q^{2}-m_{X}^{2}} \\
& \sim \frac{4 \pi}{\Lambda^{2}}
\end{aligned}
$$

then the net amplitude becomes

$$
\left|A_{Z}+A_{X}\right|^{2}=A_{Z}^{2}\left(1+\frac{A_{X}^{2}}{A_{Z}^{2}}\right)
$$



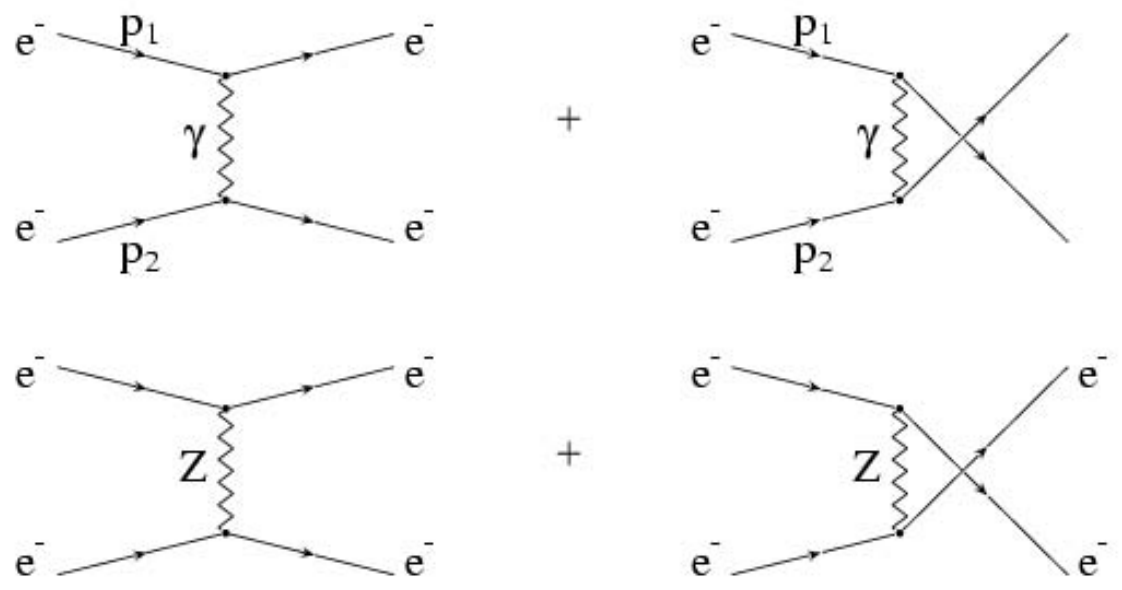

Figure 1.1: Neutral current amplitudes leading to the asymmetry $A_{L R}$ at the tree level.

Thus no interference can be observed and the sensitivity of such measurements to $X$ is suppressed. On the other hand, at low $Q^{2}\left(\ll m_{Z}^{2}\right)$, the $A_{P V}$ becomes linear in amplitudes:

$$
A_{P V} \approx A_{Z}\left(1+\frac{A_{X}}{A_{Z}}\right)
$$

Assuming that there are parity violating terms present in $A_{X}$, the measurements at low $Q^{2}$ are sensitive to other type of contact interactions.

\subsection{Left-Right Asymmetry in Moller Scattering}

The interference between electromagnetic and weak neutral current amplitudes in Fig. 1.1 gives rise to the standard model prediction of parity violating asymmetry $[14,15]$ : 


$$
A_{L R}\left(e^{-} e^{-} \longrightarrow e^{-} e^{-}\right)=\frac{G_{F} Q^{2}}{\sqrt{2} \pi \alpha} \frac{4(1-y)}{1+y^{4}+(1-y)^{4}} g_{e e}
$$

where

$$
\begin{aligned}
y & =\frac{1-\cos \theta_{c m}}{2} \\
Q^{2} & =-q^{2}=y\left(p+p^{\prime}\right)^{2} \\
& =y\left(2 m_{e}^{2}+2 m_{e} E_{\text {beam }}\right)_{\text {fixed target }}, \\
q^{2} & =\left(p^{\prime}-p\right)^{2}, \\
g_{e e} & =2 \rho g_{V} g_{A}=\left(\frac{1}{4}-\sin ^{2} \theta_{W}\right) .
\end{aligned}
$$

$\theta_{c m}$ is the scattering angle in center of mass frame and $\theta_{W}$ is the weak mixing angle. $g_{V}$ and $g_{A}$ are the vector and axial vector couplings of the electron to the $Z^{\circ}$ boson. Note that $A_{P V}=-A_{L R}$. In terms of $\theta_{c m}$ :

$$
A_{L R}=m_{e} E_{\text {beam }} \frac{G_{F}}{\sqrt{2} \pi \alpha} \frac{4 \sin ^{2} \theta_{c m}}{\left(3+\cos ^{2} \theta_{c m}\right)^{2}}\left(1-4 \sin ^{2} \theta_{W}\right)
$$

The simple functional form of the asymmetry suggests Moller scattering to be an excellent probe of the weak mixing angle at low $Q^{2}$. Note that $g_{e e}$ is close to zero since $\sin ^{2} \theta_{W} \sim 0.23$. Thus a small (relative) change in $\sin ^{2} \theta_{W}$ produces a much larger change in $A_{L R}$. Thus E158's sensitivity to $\sin ^{2} \theta_{W}$ is greatly improved by its closeness to 0.25 , and an $8 \%$ measurement of $A_{L R}$ yields $0.3 \%$ measurement of $\sin ^{2} \theta_{W}$.

For fixed target experiments, the asymmetry in (1.4.1) is very small because of the tiny $G_{F} Q^{2}$ factor and (to lesser extent) the $g_{e e}$ suppression factor. For 
$E_{\text {beam }}=50 \mathrm{GeV}$ and $\theta_{c m}=\frac{\pi}{2}$ (corresponding to final state energy of $24 \mathrm{GeV}$ ), and assuming $100 \%$ polarization, (1.4.3) yields $A_{L R}=2.97 \times 10^{-7}$. (Note that $A_{\text {measured }}=P_{b} A_{P V}$ where $P_{b}$ is the polarization of the incident beam.) The running of $\sin ^{2} \theta_{W}$ from the $Z^{\circ}$ resonance to $Q^{2}=0.025 \mathrm{GeV}^{2}$ (due to radiative corrections discussed below) reduces this prediction by $40 \%$ [15]. Including kinematic factors and the beam's $\sim 85 \%$ polarization lowers the raw asymmetry expected within the context of standard model to 140 parts per billion (ppb).

To measure such a small asymmetry to high degree of precision, both a scattering medium with a high relative cross section and an electron beam with a high luminosity are required. Since we need to achieve a statistical error close to $10^{-8}$ on $A_{P V}$, more than $10^{7}$ scattered electrons must be detected every pulse. SLAC's accelerator can deliver up to $4-6 \times 10^{11} e^{-} /$pulse at $120 \mathrm{~Hz}$ for the desired incident beam energies (45 or $48 \mathrm{GeV}$ ). For unpolarized Moller scattering the formula for the cross section is given by

$$
\frac{d \sigma}{d \Omega}=\frac{\alpha^{2}}{2 m_{e} E_{\text {beam }}} \frac{\left(3+\cos \theta_{c m}\right)^{2}}{\sin ^{4} \theta_{c m}}
$$

where $\alpha$ is the fine structure constant. For a $48.3 \mathrm{GeV}$ incident electron beam (corresponding to a $Q^{2}=0.027 \mathrm{GeV}^{2}$ ) and a spectrometer acceptance of $4.7-7.1 \mathrm{mrad}$ in the lab frame, the calculated cross section is $11.2 \mu$ barn [16]. Consequently, producing a statistical error of only a few percent in a matter of months requires the target to be quite thick. 


\subsection{Radiative Corrections and Running of $\sin ^{2} \theta_{W}$}

As discussed above, the tree level $A_{L R}$ for E158 is proportional to $1-4 \sin ^{2} \theta_{W}$ and hence suppressed because $\sin ^{2} \theta_{W} \simeq 0.23$. However, some radiative corrections are not suppressed by $1-4 \sin ^{2} \theta_{W}$ and can be potentially large. A complete calculation of radiative corrections has been carried out by Czarnecki and Marciano [15] for low $Q^{2}$ as appropriate to E158. They used modified minimal subtraction $(\overline{M S})$ scheme and defined the renormalized weak mixing angle at an energy scale of $M_{Z}$. At that energy scale, the weak mixing angle has been measured: $\sin ^{2} \theta_{W}\left(M_{Z}\right) \overline{M S}=$ $0.23073 \pm 0.00028$ [17]. The following section, adapted from [15,21], summaries the effects of the radiative corrections on $A_{L R}$ and $\sin ^{2} \theta_{W}$.

The largest one-loop radiative corrections to $A_{L R}\left(e^{-} e^{-}\right)$at low energies come from three sources:

1. $\gamma Z$ mixing and the anapole momentum ${ }^{1}$.

2. $W W$ box diagrams.

3. Photonic vertex and box diagrams.

These corrections modify (1.4.1) as [15]:

$$
\begin{aligned}
A_{L R}\left(e^{-} e^{-}\right)= & \frac{G_{F} Q^{2}}{\sqrt{2} \pi \alpha} \frac{1-y}{1+y^{4}+(1-y)^{4}}\left\{1-4 \kappa(0) \sin ^{2} \theta_{W}\left(M_{Z}\right) \overline{M S}\right. \\
& +\frac{\alpha\left(M_{Z}\right)}{4 \pi s^{2}}-\frac{3 \alpha\left(M_{Z}\right)}{32 \pi s^{2} c^{2}}\left(1-4 s^{2}\right)\left[1+\left(1-4 s^{2}\right)^{2}\right] \\
& \left.+F_{1}\left(y, Q^{2}\right)\right\},
\end{aligned}
$$

\footnotetext{
${ }^{1}$ The "anapole moment" is a parity violating electron-photon coupling that arises from higher order contributions involving weak vector bosons, such as Fig. 1.2(c).
} 


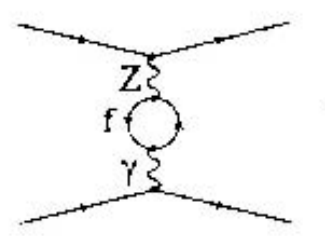

(a)

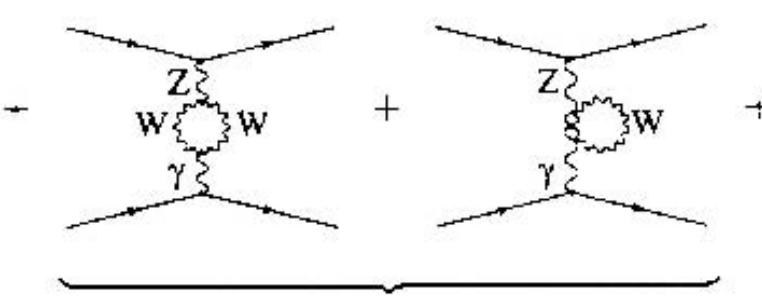

(b)

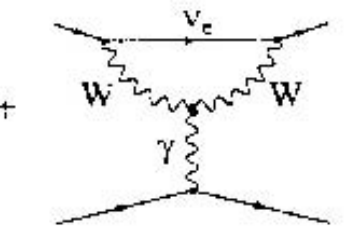

(c)

+ Inverted + crossed diagrams

Figure 1.2: $\gamma Z$ mixing diagrams (a) and (b), $W$-loop contribution to anapole moment (c).

where

$$
\begin{aligned}
s & \equiv \sin ^{2} \theta_{W}\left(M_{Z}\right)_{\overline{M S}} \\
c & \equiv \cos ^{2} \theta_{W}\left(M_{Z}\right)_{\overline{M S}} .
\end{aligned}
$$

The diagrams in Fig. 1.2 with $\gamma Z$ mixing and the anapole moment produce the largest effect. It effectively replaces the tree level $1-4 \sin ^{2} \theta_{W}$ in $A_{L R}$ by:

$$
1-4 \kappa(0) \sin ^{2} \theta_{W}\left(M_{Z}\right)_{\overline{M S}}
$$

where

$$
\kappa(0)=1.0301 \pm 0.0025
$$

represents a $3 \%$ shift in the effective $\sin ^{2} \theta_{W}$ due to loop effects. That $+3 \%$ increase in $\sin ^{2} \theta_{W}$ appropriate for low $Q^{2}$ gives rise to a $38 \%$ reduction in $A_{L R}$. This 


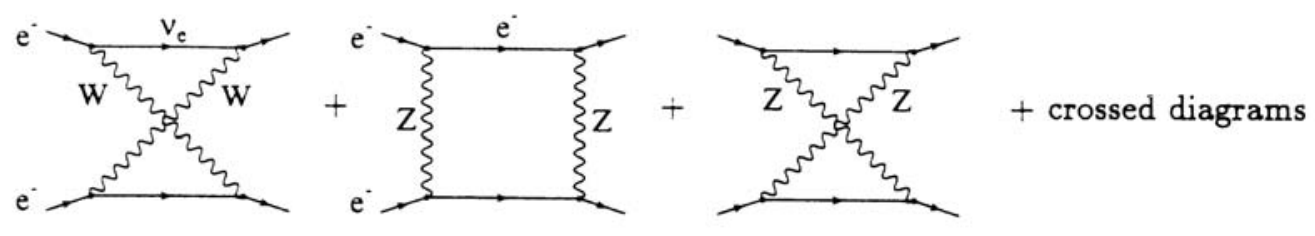

Figure 1.3: Box diagrams with two heavy bosons.

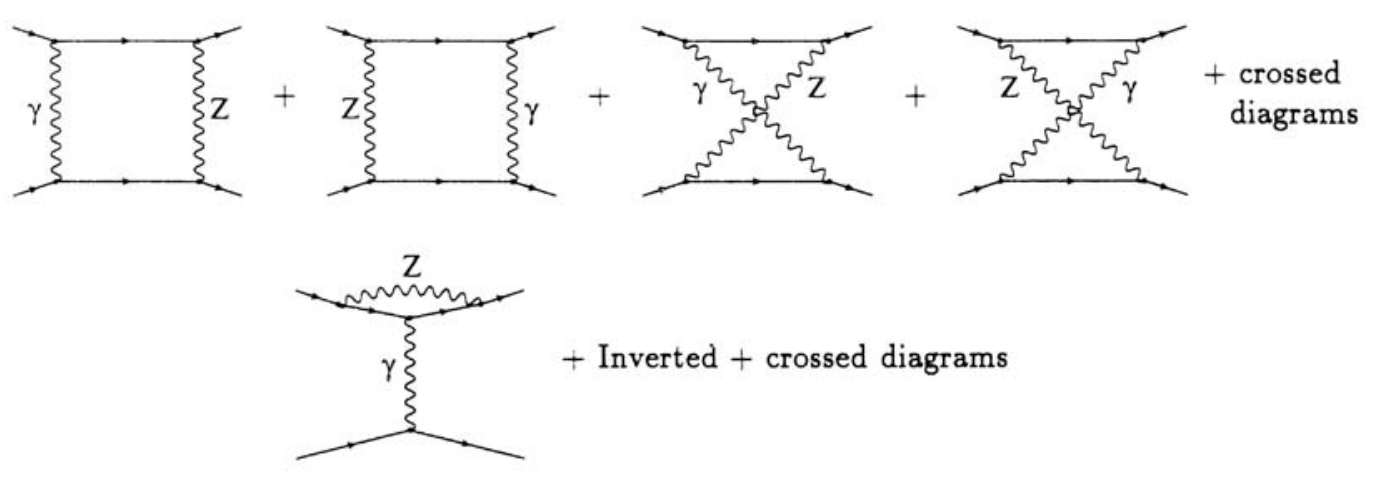

Figure 1.4: Boxes containing one photon and $Z$-loop contribution to the anapole moment.

reduction actually makes E158 more sensitive to $\sin ^{2} \theta_{W}\left(M_{Z}\right) \overline{M S}$ as well as "new physics".

The next source of one-loop corrections comes from the $W W$ and $Z Z$ box diagrams in Fig. 1.3. The $W W$ box is not suppressed by $1-4 s^{2}$ and gives rise to the term $\alpha\left(M_{Z}\right) / 4 \pi s^{2}$ term in (1.5.1). Taken alone, this gives a $4 \%$ enhancement of $A_{L R}$ relative to the lowest order prediction. The $Z Z$ box diagrams are suppressed by $1-4 s^{2}$. Hence their contribution, the $3 \alpha\left(M_{Z}\right)\left(1-4 s^{2}\right)\left[1-\left(1-4 s^{2}\right)^{2}\right] / 32 \pi s^{2} c^{2}$ term in (1.5.1), is tiny, $\sim 0.1 \%$.

The next set of loops is illustrated in Fig. 1.4. Together with photonic corrections to the external legs and vertices in Fig. 1.1, they give rise to $Q^{2}$ dependent 
corrections denoted by $F_{1}\left(y, Q^{2}\right)$ in $(1.5 .1)$. For $Q^{2}=0.02 \mathrm{GeV}^{2}[15]$ :

$$
F_{1}\left(1 / 2,0.02 \mathrm{GeV}^{2}\right)=-0.0041
$$

Collecting all the one-loop radiative corrections, one finds, for $y=1 / 2$ and $Q^{2}=0.027 \mathrm{GeV}^{2}$ :

$$
1-4 \sin ^{2} \theta_{W}=0.0744 \longrightarrow 0.0450 \pm 0.0023 \pm 0.0010 .
$$

The first error arises from hadronic loops in $\gamma Z$ mixing diagrams and the second from uncertainty in the photonic corrections to the external legs and vertices of Fig. 1.1.

The result in (1.5.3) represents a $40 \pm 3 \%$ reduction in the asymmetry because of quantum loop effects. For $y=1 / 2$ and $Q^{2}=0.027 \mathrm{GeV}^{2}$, one finds that the radiative corrections reduce $A_{L R}\left(e^{-} e^{-}\right)$from $2.97 \times 10^{-7}$ to $1.80 \times 10^{-7}$.

The predicted "running" of $\sin ^{2} \theta_{W}$ from the value obtained at $Q^{2}=M_{Z}^{2}$ in measurements obtained by SLC and LEP $[18,19]$ is shown in Fig. 1.5. It is this running that E158 seeks to establish with high precision.

In summary, because Moller scattering is a fully leptonic process, its one-loop radiative corrections can be calculated with high precision. E158 provides a similarly precise measurement of those radiative corrections, thus testing the electroweak sector of the standard model at the quantum loop level.

\section{6 "New Physics" Sensitivity}

A high precision $A_{L R}$ measurement away from the $Z$ pole can be utilized to search for or constrain "new physics". A disagreement with the extracted $\sin ^{2} \theta_{W}\left(M_{Z}\right) \overline{M S}$ 


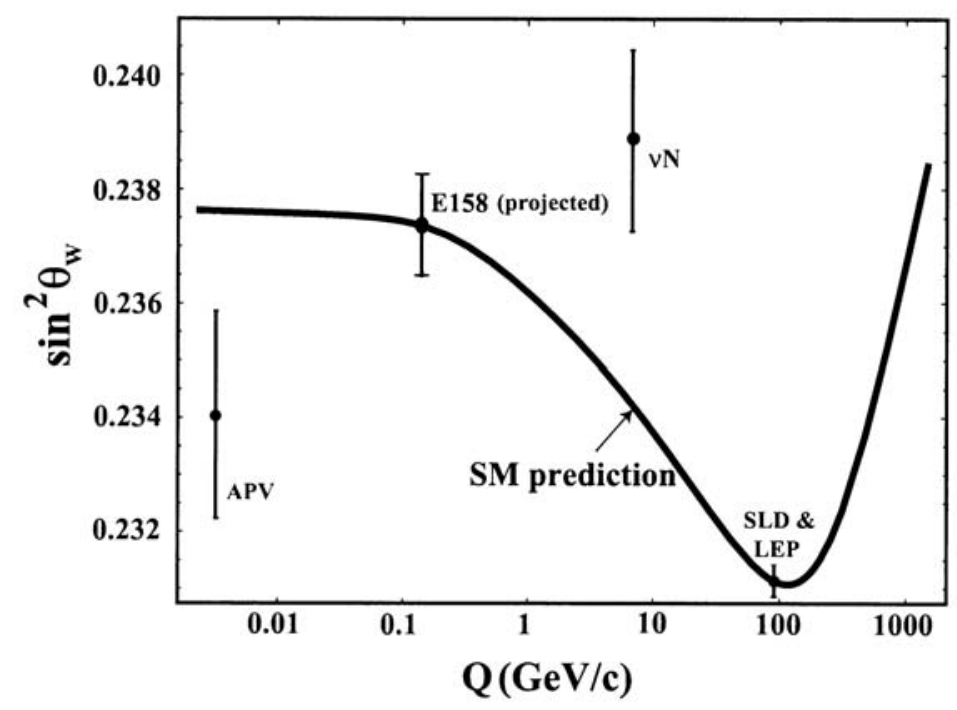

Figure 1.5: The solid curve shows the predicted running of $\sin ^{2} \theta_{W}\left(Q^{2}\right)$ from the precision measurement at the $Z^{\circ}$ resonance $[20,21]$. APV refers to the measurement of the parity violation in atomic $\mathrm{Cs}[22]$, and $\mathrm{NuTeV}$ results come from [23].

value from $Z$ pole determinations could signal the presence of additional tree or loop level neutral current effects. $A_{L R}$ can indicate the deviation from the Standard Model but cannot specify the source. Examples of new physics scenarios that have been discussed in the literature include $Z^{\prime}$ bosons, compositness, doubly charged scalars $\Delta^{++}$etc.

\subsection{1 $Z^{\prime}$ Bosons}

Many extensions of the standard model predict new interactions at the $\mathrm{TeV}$ scale. If the interaction is mediated by a new neutral gauge boson $Z^{\prime}$ which does not mix significantly with the $Z^{\circ}$, it could have escaped detection in past experiments. E158's measurement is sensitive to parity violating weak neutral current interac- 
tions involving left and right handed electron currents of the form:

$$
\sigma_{L}-\sigma_{R} \propto\left(\bar{\psi}_{L} \gamma^{\mu} \psi_{L}\right)^{2}-\left(\bar{\psi}_{R} \gamma^{\mu} \psi_{R}\right)^{2} \Longrightarrow \delta\left(A_{L R}\right) \propto \frac{e^{2}\left(Q_{L}^{2}-Q_{R}^{2}\right)}{M_{Z^{\prime}}^{2}}
$$

where $\psi_{R, L}$ and $\bar{\psi}_{R, L}$ are electron chiral spinors and $e Q_{R}$ and $e Q_{L}$ are the chiral couplings of the electron to the $Z^{\prime}$ boson.

One can quantify the sensitivity of the measurement to a new interaction by evaluating the $Z^{\prime}$ mass $M_{Z^{\prime}}$ for which the experimental measurement differs from the theoretical prediction by 2 standard deviations. If the E158 result for $\sin ^{2} \theta_{W}$ is within $2 \sigma$ from the predicted standard model value, a lower limit of 600 to 900 $\mathrm{GeV}$ could be set on the mass of the $Z^{\prime}$ (for certain theoretical models) [4]. As the Tevatron should be capable of seeing $Z^{\prime}$ with a mass up to $1 \mathrm{TeV}$, E158 will provide a strong complementary result.

\subsubsection{Other Contact Interactions}

E158 is also sensitive to other types of new contact interactions. Electron compositeness can be parameterized as a contact interaction with a Lagrangian of the form $[24]$

$$
\mathcal{L}=\frac{4 \pi}{2 \Lambda_{e e}^{2}}\left[\eta_{L L}\left(\bar{\psi}_{L} \gamma_{\mu} \psi_{L}\right)^{2}+\eta_{R R}\left(\bar{\psi}_{R} \gamma_{\mu} \psi_{R}\right)^{2}+2 \eta_{L R}\left(\bar{\psi}_{R} \gamma_{\mu} \psi_{R}\right)\left(\bar{\psi}_{L} \gamma_{\mu} \psi_{L}\right)\right]
$$

where $\Lambda_{e e}$ is the energy scale at which the internal dynamics of the electron become important and $\left|\eta_{i f}\right| \leq 1$. If the contact interaction possesses a parity violating term then E158 can have a large sensitivity to it:

$$
g_{e e}(\text { meas. })-g_{e e}(\mathrm{SM})= \pm \frac{\pi}{G_{F} \sqrt{2}} \frac{\eta_{R R}-\eta_{L L}}{\Lambda_{e e}^{2}}
$$


For $\eta_{R R}$ or $\eta_{L L}$ equal to \pm 1, E158 is sensitive to electron compositness at energy scale up to $14 \mathrm{TeV}$. Current limits on $\Lambda_{e e}$ from $\mathrm{e}^{+} \mathrm{e}^{-}$colliders are in the range of 1 to $3 \mathrm{TeV}$ [25]. Lepton-flavor violating processes such as exchange of a doubly charged Higgs boson $\Delta^{++}$, can also be probed by E158 with level of sensitivity an order of magnitude greater than current indirect constraints.

\subsubsection{Oblique Corrections}

Very massive particles that do not couple to the electron at tree level can manifest themselves by modifying the low-energy coupling constants through contributions in higher order loop diagrams. These changes to the low energy coupling constants are referred to as "oblique corrections" [26,27]. For new physics at mass scale much greater than $M_{Z}$, only three parameters are needed to describe oblique corrections [28], and they are called $S, T$ and $U$. Only $S$ and $T$ affect $Z^{\circ}$ pole observables $[28,29]$, and they are now tightly constrained by LEP and SLC measurements. For new Physics at mass scales down to $M_{Z}$, additional three parameters called $V$, $W$, and $X$ are needed $[30,31]$. The parameter $X$ can be interpreted as a measure of the running of $\sin ^{2} \theta_{W}$ due to physics beyond the Standard Model. It can be approximated as

$$
\sin ^{2} \hat{\theta}_{W}\left(M_{Z}^{2}\right)-\sin ^{2} \hat{\theta}_{W}(0) \simeq \alpha X
$$

where $\alpha$ is the fine structure constant. The current world average is $X=0.38 \pm 0.51$ [32]. E158 would be sensitive to $X$ at the level of 0.15 . If $X$ is nonzero it would indicate that the mass scale for new physics is not muck higher than $M_{Z}$ and that the new physics does not couple strongly to the $Z^{\circ}[4]$. 
18 Parity Violating Asymmetries in Polarized Electron Scattering 


\section{Chapter 2}

\section{Experimental Design}

\subsection{Experimental Considerations}

The polarized source at SLAC generates $2-6 \times 10^{11}$ electrons per pulse with $80 \%$ beam polarization at a repetition rate of $120 \mathrm{~Hz}$ with the ability to assign the sign of the beam helicity on a pulse-to-pulse basis. The experimental asymmetry is measured by rapidly flipping between the two possible electron beam helicity states while keeping all other experimental parameters virtually unchanged and then averaging the fractional difference in the cross section over many such complementary pairs of beam pulses.

The critical requirement in an asymmetry measurement is to keep the differences in the beam characteristics between left- and right-handed pulses to a negligible level. Another important aspect is the reversal of the sign of the physics asymmetry by several independent methods. 


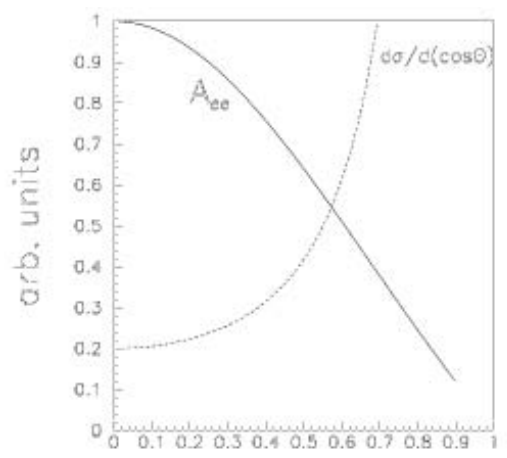

$|\cos \Theta|$

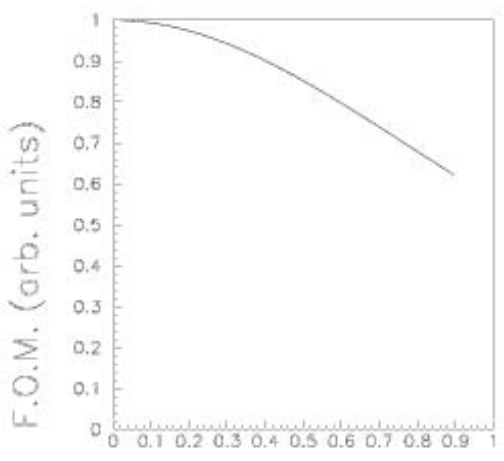

$|\cos \theta|$

Figure 2.1: The behavior of the asymmetry, the differential cross section and the figure of merit as a function of $\left|\cos \theta_{c m}\right|$.

\subsubsection{Figure of Merit}

The asymmetry is maximal at $E^{\prime}=25 \mathrm{GeV}\left(\cos \theta_{c m}=0\right)$, and falls to zero at $E^{\prime}=0$ and $50 \mathrm{GeV}$ as shown in Fig. 2.1(a). For the experimental design, an important parameter is the figure-of-merit (f.o.m.), which quantifies the variation of achievable statistical error for fixed luminosity. For E158, the f.o.m. is proportional to $A_{P V}^{2} \times \frac{d \sigma}{d \Omega}$, and its dependence on the center of mass angle goes like $\left(3+\cos ^{2} \theta_{c m}\right)^{-2}$. It can be seen in Fig. 2.1(b) that f.o.m. varies slowly with $\cos \theta_{c m}$ and is relatively flat in the range $-0.5<\cos \theta_{c m}<0$. Consequently, the experiment is tuned to accept particles in this range.

\subsubsection{Target}

Since we need to achieve a statistical error close to $10^{-8}$ on $A_{P V}$, more than $10^{7}$ scattered electrons must be detected every beam pulse. (The expected rate is between $2 \times 10^{7}$ and $4 \times 10^{7}$ electrons per pulse.) Liquid hydrogen is the natural choice to provide a dense electron target. It provides the least amount of radiation 
loss for a given target thickness. Furthermore, the dominant background for a hydrogen target at $Q^{2} \sim 0.02 \mathrm{GeV}^{2}$ is elastic electron-proton scattering that is well understood and has a small electroweak asymmetry. In order to achieve the necessary rate, one needs $10 \mathrm{gm} / \mathrm{cm}^{2}$ of liquid hydrogen, which is approximately $150 \mathrm{~cm}$ long.

\subsubsection{Integration}

At the high electron scattering rate as mentioned earlier, integrating the signal over the duration of the beam pulse is most practical. Integration allows one to use a relatively simpler detector and Data Acquisition System (DAQ) package and eliminates dead time problems and hence potentially dangerous corrections for helicity-correlated dead time. One of the challenges of the integration technique is that it provides no opportunity to identify and reject background events. This requires that the elastically scattered electrons be focused into a region free of background into a total absorption shower counter. In addition, integration places stringent requirements on the linearity and resolution of the detectors' readout electronics.

\subsubsection{Spectrometer}

For Moller scattering, there is kinematical correlation between the scattered electron energy $E^{\prime}$ and scattering angle. The range of scattering angles for $10<E^{\prime}<$ $40 \mathrm{GeV}$ is 2.25 to $9 \mathrm{mrad}$ in the lab frame. The scattered electrons with the highest asymmetry are those, which have scattered at 90 degrees in the center of mass frame which corresponds to $E^{\prime}=24 \mathrm{GeV}$. Since we are dealing with identical particles, a good event generates an electron at an azimuthal angle $\phi$ with energy $E^{\prime}$ 
simultaneously with another electron at an azimuthal angle of $\pi-\phi$ with energy $E-E^{\prime}$. The full available solid angle in the azimuth for scattered electrons from 10 to $40 \mathrm{GeV}$ is thus obtained by detecting 10 to $25 \mathrm{GeV}$ electrons over $2 \pi$ radians in $\phi$. The spectrometer is required to accept the required range of scattered electrons while rejecting against elastic electron-proton scattering, the dominant high energy background.

A large percentage of the beam energy (around 16\%) will be converted into photons, so the spectrometer must be able to block this background (which could hit the detector) while allowing the majority of the flux to travel unimpeded to the beam dump. The simplest method of blocking the photon background is through the use of dipole chicane. Three diples can be used to bend all charged particles away from the beam axis, allowing the forward photons to be collimated.

While passing through the chicane, the electron beam radiates quite a bit of synchrotron radiation. This radiation occurs in the horizontal plane, but it must be blocked, as it would place (if it were not collimated) as much power on the Moller detector as the Moller signal flux.

Once the beam has been steered through the chicane, the $e P$ flux must be separated from the Moller flux. On average, the $e P$ elastic scatters are at momenta very close to the beam momentum $(48 \mathrm{GeV})$, whereas the desired Moller scatters are lower than $24 \mathrm{GeV}$. The easiest way to separate the two signals is to remove a radial slice from the distribution and then magnetically focus the lower momentum Mollers into that empty region. Therefore, immediately after the dipole chicane, there must be a radial collimator, followed by quadrupole focusing optics. Furthermore, the Moller detector should be located as far from the target as possible so that the Moller/eP radial separation at the detector is maximized. 


\subsubsection{Beam Monitoring}

The extremely small scattering angle of the experiment, combined with the strong dependence of the scattering cross section on the angle, causes the detector asymmetry to be very sensitive to helicity correlations in the beam parameters. Consequently, all pertinent beam parameters must be measured with a system of very precise beam monitors. Assuming that the Moller asymmetry width is about 150 ppm, each monitor's resolution should ideally be high enough such that, when beam asymmetries are subtracted from the detector asymmetry, the overall contribution to the width of the final asymmetry from each monitor is no more than $30 \mathrm{ppm}$.

\subsubsection{Feedbacks}

The correlated pulse to pulse differences in the beam characteristics can induce false asymmetries in the experiment. Most of the false asymmetries due to change in beam characteristics with helicity reversal can be traced back to helicity correlations of the laser intensity and position at the polarized source. The overall intensity and position asymmetries can be suppressed to a negligible level by implementing active feedback loops coupling the beam asymmetries to the intensity and position of the source laser.

\subsection{Physics Backgrounds}

The spectrometer and the detector geometry have been carefully chosen so that the only relevant backgrounds are those that are produced in the same range of laboratory scattering angles that corresponds to the range of scattering Moller 
electrons of interest. These background processes include inelastic electron-proton scattering of beam energy electrons, real and virtual photo-production of pions and synchrotron radiation.

\subsubsection{Electron Background}

With a liquid hydrogen target, the largest background under the Moller peak will come from the scattering of electrons off the protons. The primary concern from the elastic $e P$ background is not only the rate, but that overall elastic $e P$ asymmetry has approximately the same value as the Moller asymmetry. Although the spectrometer is optimized to separate Moller and $e P$ scatters, there is still significant leakage of the $e P$ into the Moller region, due to interactions in which the electron radiates before the initial scattering off the proton.

Other electron background to the Moller scattering comes from the inelastic scattering of beam electrons with the full beam energy from the protons in the target. The cross section for this process is quite small as compared to the Moller cross section, and contributes on the order of $2 \%$ [4] of the flux in the Moller kinematic region. The asymmetry for this process, though, is much larger than either the elastic $e P$ or the Moller asymmetry. This asymmetry is not well known theoreti-

cally, primarily because calculations of the background involves both resonant and non-resonant processes, most of which have not been measured with any accuracy. The prediction for the magnitude of the inelastic asymmetry, $A_{P V}=10^{-4} Q^{2}$, is an estimate based on the asymmetry of processes below and at the delta resonance. For a $Q^{2}$ of $0.03 \mathrm{GeV}^{2}$, this asymmetry ends up at $3 \mathrm{ppm}$. Any measurement of the Moller asymmetry will require, as a correction, an extremely accurate measurement of the elastic and inelastic $e P$ distribution in the Moller detector. 


\subsubsection{Pions}

There are two primary sources of pion background: photo-production and deep inelastic scattering. High energy pions, unlike the electrons, can punch through the collimators. As estimated in [4], the pions from real photo-production dilutes the asymmetry by at most $0.5 \%$, and the estimate for the asymmetry correction is $1.5 \%$. The dilution from pion virtual photoproduction is negligible: $<2 \times 10^{-3}$ [33]. The asymmetry correction on the other hand is larger than that for real photoproduction and is estimated to be $1.3 \%$ [33].

Most important pion background comes from the pions produced in the deep inelastic scattering process. This background is potentially dangerous because the electroweak asymmetry is three orders of magnitude bigger than the Moller asymmetry. The estimated dilution factor from these pions is $2 \times 10^{-4}$ and the asymmetry correction is $1 \%$ [4].

The overall uncertainty in the asymmetry and relative rates of all three pion processes stems from a lack of a precision pion measurement in this kinematic region (mainly because the signal is swamped by Moller electrons). To accurately estimate the pion contribution to the Moller asymmetry, these pions must be measured; in other words, E158 must contain a pion detector capable of distinguishing between pions and other charged flux.

\subsubsection{Synchrotron Radiation}

Another potential correction to the Moller asymmetry comes from synchrotron photons, because they can acquire an asymmetry if the beam has any transversely polarized electrons $[34,35]$. Although, the E158 beam should be polarized purely longitudinally, vertical magnets present in the beamline before the beam reaches 
the E158 spectrometer can impart some small amount of transverse polarization to the beam. This polarization can affect the measured beam asymmetry in one of the two ways. First, synchrotron light emitted in the large bend before the E158 spectrometer can leave an overall asymmetry present in the beam which is uncorrelated with other beam parameters. A synchrotron light monitor [36] has been placed upstream of the E158 spectrometer to measure this effect. Second, synchrotron light emitted in the E158 spectrometer can strike the Moller detector. If this light carries a large asymmetry, it might contribute to the measured Moller asymmetry.

\subsubsection{Neutral Background}

Aside from backgrounds with high asymmetries, the Moller detector is susceptible to neutral backgrounds from variety of sources. When the electron beam passes through the target, approximately $16 \%$ of the beam energy converts to prompt and secondary photons from interactions in the target. Photons which scatter at wide angles must be blocked, so as to prevent dilution of the Moller asymmetry. A second source of neutral background is from showers occuring at the inner edges of the momentum and photon collimators.

The third and final source of neutral background is neutral hadrons, which can originate from anywhere in the spectrometer, as well as from downstream sources such as the beam dump. This flux is expected to be quite small, particularly due to the small potential for interactions within the Moller detector. 


\subsection{Estimate of Rate}

The scattering cross section can be estimated by integrating equation 1.4.4 over the scattering angle acceptance of the spectrometer $\left(65.9^{\circ}<\theta_{c m}<89.4^{\circ}\right.$, and assuming $2 \pi$ acceptance in azimuth) and assuming a beam energy of $48.3 \mathrm{GeV}$. We find $\sigma \approx 11.2 \mu$ barn [37]. The number of detected scattered electrons per pulse, $N_{s}$, can then be estimated as

$$
N_{s}=\sigma I \rho L f_{s}
$$

where $I$ is the beam current, $\rho$ is the target density, $L$ is the target length, and $f_{s}$ is a correction factor to account for losses due to collimators that block synchrotron radiation in the horizontal plane. Using the values [37]

$$
\begin{aligned}
\sigma & =11.2 \mu \text { barn }, \\
I & =3.5 \times 10^{11} \text { electrons } / \text { pulse }, \\
\rho & =0.072 \mathrm{~g} / \mathrm{cm}^{3}=4.3 \times 10^{22} \text { electrons } / \mathrm{cm}^{3}, \\
L & =150 \mathrm{~cm}, \\
f_{s} & =0.89,
\end{aligned}
$$

we find that the rate into the detector should be 22.5 million electrons per pulse, or $2.7 \mathrm{GHz}$ at $120 \mathrm{~Hz}$. Thus, we expect the pairwise asymmetry distribution to have a width of $\sim 1 / \sqrt{45 \times 10^{6}}=150 \mathrm{ppm}$. 


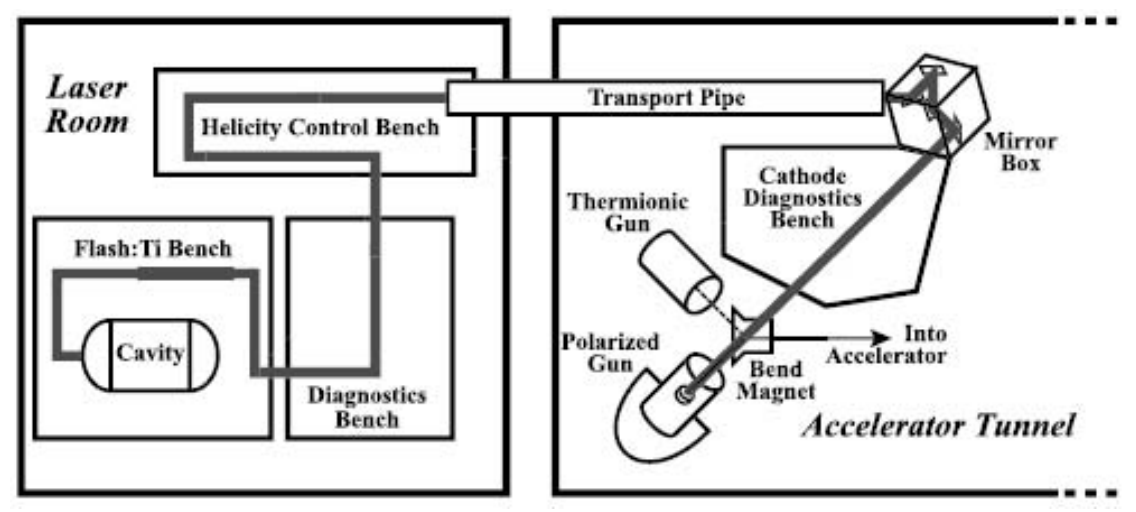

Figure 2.2: An overview of the Polarized Electron Source as it is configured for E158.

\subsection{Polarized Electron Source}

To achieve the proposed systematic error, E158 requires an incredibly stable beam with an intensity of $6 \times 10^{11}$ electrons per spill and with stringent demands on the beam position and charge asymmetries and beam jitter. SLAC beam hardware (source and accelerator) as described below, is capable of delivering such a stable beam.

The SLAC polarized electron source went through significant upgrades in preparation for E158 [38]. The source is based on photo emission from strained GaAs or GaAsP cathode pumped by an intense, circularly polarized laser beam. E158 uses a flashlamp-pumped Ti:Sapphire laser (the "Flash:Ti") made by Big Sky Laser Technologies, which emits an $805 \mathrm{~nm}$ beam at frequency of $120 \mathrm{~Hz}$ (the rate at which the accelerator deliver pulses). Table 2.1 summarizes the parameters of the Flash:Ti laser beam for E158. The polarized source laser and optics systems are housed in an environmentally controlled room outside of the accelerator tunnel. An overview of the laser and optics systems as they are configured for E158 is illus- 


\begin{tabular}{|l|c|}
\hline \hline Wavelength & $805 \mathrm{~nm}$ \\
\hline Bandwidth & $0.7 \mathrm{~nm} \mathrm{FWHM}$ \\
\hline Repetition rate & $120 \mathrm{~Hz}$ \\
\hline Pulse Length & $270 \mathrm{~ns}$ \\
\hline Pulse energy & $60 \mu \mathrm{J}$ \\
\hline Circular Polarization & $99.8 \%$ \\
\hline Intensity Jitter & $0.5 \% \mathrm{rms}$ \\
\hline Position Jitter at Photocathode & $<70 \mu \mathrm{rms}$ \\
\hline
\end{tabular}

Table 2.1: Parameters of the Flash:Ti laser beam (for E158 2002 Physics Run I).

trated in Fig. 2.2. The "Flash:Ti Bench" holds the laser cavity and pulse-shaping optics. The "Diagnostic Bench" has photodiodes for monitoring the laser's intensity and temporal profile and a monochromator for measuring its wavelength. The "Helicity Control Bench" houses the optics for controlling the polarization state of the beam and for suppressing beam asymmetries. A $20 \mathrm{~m}$ Transport Pipe transports the beam into the accelerator tunnel, where it crosses the "Cathode Diagnostics Bench" and is directed onto the cathode of the polarized gun. The Cathode Diagnostics Bench holds optics for setting the position of the beam spot on the cathode and an auxiliary diagnostic line. The photoelectrons emitted by the cathode are bent through $38^{\circ}$ and enter the accelerator.

\subsubsection{Circular Polarization}

The Helicity control bench contains the optics that circularly polarize the laser beam in a manner that permits selecting the helicity in a psudorandom sequence 
Figure 2.3: The Helicity

Control Bench contains the

optics for control of the

laser beam polarization and

beam asymmetries.

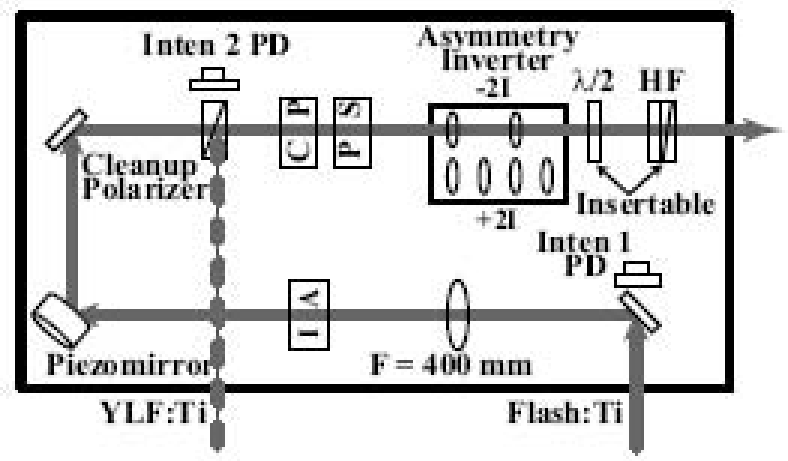

on pulse-to-pulse basis. Fig. 2.3 shows major components of the bench which are used to control the laser beam's polarization.

The polarization optics are designed to generate highly circularly polarized light of either helicity while minimizing the beam asymmetries. The "Cleanup Polarizer", and "Circular Polarizer" (CP) and the "Phase Shift" (PS) Pockels cells collectively determine the polarization of the beam. The CP cell acts as a quarterwave plate with its fast axis at $45^{\circ}$ from the horizontal. The sign of its retardation can be chosen on a pulse-by-pulse basis, generating circularly polarized light of either helicity. Adjusting its voltage from the "quarter-wave setting" allows the $\mathrm{CP}$ cell to compensate for linear polarization along the horizontal and vertical axes that arises from imperfections in the source optics. Even a small amounts of linear beam polarization can lead to large position and intensity asymmetries in the beam. The PS cell, with a vertical fast axis, is similar to the CP cell and is used to compensate for the residual linear polarization along the axes at $\pm 45^{\circ}$.

\subsubsection{Insertable Half Wave Plates}

One of the most powerful tools for negating systematics in parity experiments is the ability to change the sign of the physics asymmetry independently of any 
other experimental parameter. By doing so, limits can be placed on the size of systematic helicity correlations affecting the physics asymmetry. E158 contains three such potential flips, two built into the source. The first of the two is an insertable zeroth order halfwave plate.

There are two halfwave plates in the optics system following the polarization optics that can be used to introduce a slow reversal of the laser helicity. This flips the definition of helicity relative to what the DAQ is expecting, thus reversing the sign of the physics asymmetry. One halfwave plate is located on the Helicity Control Bench, and the second is located in the Cathode Diagnostic Bench immediately before the cathode. Either half wave plate can be used to affect the slow reversal, but the one in the Cathode Diagnostic Bench was used during the experiment. In reality, the halfwave plate introduces some additional intensity asymmetry due to imperfections in the crystal (which introduces linear polarization into the laser beam).

\subsubsection{Asymmetry Inverter}

The second device for changing experimental asymmetries is the "asymmetry inverter". This is a system of four lenses, mounted in series and located immediately before the halfwave plate in the Helicity Control Bench. Ideally, these lenses perform exactly the opposite function as the halfwave plate: they invert the position and intensity asymmetries while leaving the experimental asymmetry unchanged. Like the halfwave plate, though, these lenses might introduce additional intensity (and position) asymmetries into the beam; this will be judged during the analysis.

The third method of asymmetry inversion involves changing the beam energy which will be discussed later. 


\subsubsection{Cathode}

After the laser light passes through the source optics, it strikes a strained GaAs cathode. To achieve the necessary luminosity $\left(6 \times 10^{11}\right.$ electrons per pulse), E158 used a new gradient-doped strained GaAsP cathode which incorporates several more layers than standard GaAs cathodes [40]. With the available laser power this cathode can yield a charge of $2 \times 10^{12}$ electrons in $100 \mathrm{~ns}$. This is significantly more charge than required by E158 (and significantly more than yielded by previous cathodes), providing additional flexibility in optimizing the optics system. Additionally, like the prior cathodes, this cathode gives a very high (80\%) polarization, which was required for the proposed experimental running time. (The overall asymmetry error is directly proportional to the polarization.)

\subsubsection{Helicity Sequence}

If there is an imbalance between the number of left handed pulses following right handed pulses and vice versa, hysteresis could introduce large helicity correlations in the beam. Accordingly, the polarization sequence is required to be random enough to negate this possibility. A SLAC-built custom electronics module located at the laser source called PMON (Polarization MONitor) generates a psudorandom helicity sequence for the beam using a 33-bit shift register algorithm as described in [39]. At $120 \mathrm{~Hz}$, this sequence repeats approximately once every two years. Because the dominant noise in the electronic environment surrounding the accelerator is at $60 \mathrm{~Hz}$, the $120 \mathrm{~Hz}$ triggering is treated as two separate $60 \mathrm{~Hz}$ time slots. This is done by imposing a "quad" structure on the helicity sequence in which two consecutive pulses have randomly chosen helicities and the subsequent two pulses are chosen to be their complements (an $A B \bar{A} \bar{B}$ pattern). In the data 
analysis, asymmetries are calculated for each pair of events, where pairs are formed between the first and third members of the quadruplet, and between the second and fourth member. In this way, the pairwise asymmetries are calculated between pulses that are at the same phase with respect to the $60 \mathrm{~Hz}$ noise. The psudorandom sequence also provides a means of error checking in the offline analysis. Observing the helicity state of 33 consecutive pairs allows one to predict the state of future pairs. Comparing the predicted state with the actual state transmitted to the DAQ can be used to look for data acquisition errors. PMON determines the pulse sequence, sets the appropriate voltages for all helicity-correlated devices (the CP, PS, and IA Pockels cells and the piezomirror), and distributes the helicity information and pulse identification number to the DAQ.

\subsection{Accelerator}

Electrons exiting the source are immediately sent into the accelerator which is made up of roughly $300 \mathrm{RF}$ cavities distributed over a two mile accelerating structure. The accelerator is divided into 28 sectors of klystron groups, each group containing eight klystrons. Each klystron consists of RF copper cavities into which 65 megawatts of RF power is pulsed at a frequency of $2856 \mathrm{MHz}$. SLAC's accelerator can achieve a theoretical maximum beam energy of $51 \mathrm{GeV}$, slightly higher than E158's requirement of $48.3 \mathrm{GeV}$. It is capable of a peak repetition rate of 120 pulses per second, and its timing system provides sufficient flexibility to divide these 120 pulses between multiple beams. The beam can be provided by either a polarized electron gun or a thermionic (unpolarized) gun. The "Injector" possesses diagnostics that were useful for commissioning E158's beam and monitoring beam asymmetries at the electron source. The Injector also contains elements that pre- 
pare each beam pulse for acceleration by appropriately bunching it to match the accelerator's $2856 \mathrm{MHz}$ RF power structure. Each sector of the accelerator also contains steering magnets (one dipole and one quadrupole), necessary for keeping the E158 beam and the PEP beam (for BaBar experiment) aligned.

The Accelerator Structure SETup (ASSET) region, at which the beam energy is $1 \mathrm{GeV}$, is a several meters long region of the accelerator in which test setups can be placed. It is often used to test advanced RF accelerator cavity designs. E158 used ASSET as a low energy diagnostic point for beam properties and beam asymmetries.

At the end of the accelerator, the Beam Switch Yard (BSY) kicks individual beam pulses into one of several beam lines: positron and electron beam lines for either the SLD interaction point or BaBar, and electron beam lines for End Station A (ESA) and End Station B (ESB). In addition, the "Final Focus Test Beam" is used as a test bed for magnetic optics for the Next Linear Collider and for experiments testing novel means of electron acceleration. E158 occupied ESA.

\subsubsection{A-Line}

Following the BSY, the electron beam must bend through $24^{\circ}$ to enter the ESA, where E158's spectrometer and detectors are located. To create such a large bend a series of twelve dipole and twelve quadrupole magnets, collectively known as "ALine", are used to steer the beam. Between the 6th and 7th dipole (at the point of highest dispersion) rest two large collimators, called the "momentum slits". These collimators can be brought as close together as possible to ensure a very narrow final beam momentum spectrum. For E158, they were set to collimate electrons to have an energy within $1 \%$ of the nominal beam energy. 
The A-Line gives E158 its final option for an experimental asymmetry sign change. The g-2 spin precession in the A-Line flips the polarization of the electron beam by $180^{\circ}$ provided the beam energy is raised from $45.0 \mathrm{GeV}$ up to $48.3 \mathrm{GeV}$. Consequently, the E158 data set is taken at these two beam energies (the highest two energies available at SLAC at which the electron polarization is longitudinal in ESA). Ideally, if the two data sets from each energy are compared, the physics asymmetry should perfectly flip sign from one to the other. In practice, the $Q^{2}$ acceptance of the spectrometer is slightly different for the two energies, and this acceptance will have small but measurable effect on the asymmetry.

\subsubsection{Beam Rate and Spills}

For E158, the beam can be run at several rates, but for the majority of the experiment, the beam is run at either 30,60, or $120 \mathrm{~Hz}$. In the very beginning of the experiment, ten million pairs of data were taken at $60 \mathrm{~Hz}$. For the remainder of the experiment, the beam was delivered at $120 \mathrm{~Hz}$. These numbers are slightly higher than the actual delivered rate due to two necessary sources of loss. First, while $\mathrm{PEP}$ is running, it always takes $2 \mathrm{~Hz}$ of beam pulses (in one time slot of $60 \mathrm{~Hz}$ ), called the witness pulses, to be used to maintain proper steering through the PEP rings. Although these pulses wind up in the E158 spectrometer, they are unpolarized and are cut from the data in the analysis. Second, $1 \mathrm{~Hz}$ of beam pulses (at $120 \mathrm{~Hz} ; 0.5 \mathrm{~Hz}$ at $60 \mathrm{~Hz}$ ) are pedestal pulses: they contain no electrons, and they are used for pedestal subtraction. At $120 \mathrm{~Hz}$ running, each time slot is missing $0.5 \mathrm{~Hz}$ of beam due to pedestals. Consequently, at "120 Hz" running, E158 typically receives $117 \mathrm{~Hz}$ of beam. 


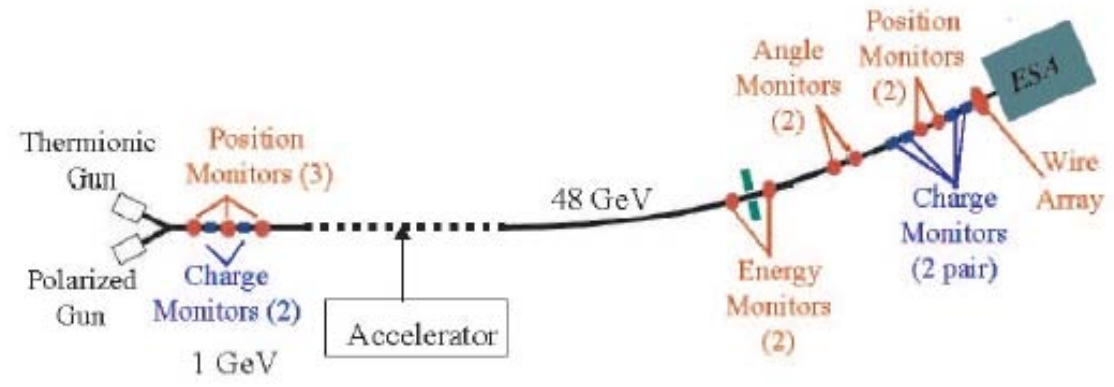

Figure 2.4: Beam Diagnostics for E158.

\subsection{Beam Monitoring}

E158 requires very precise monitoring of all beam parameters, since quite a number of systematic errors from beam asymmetries could effect the physics asymmetry. The devices used to monitor the beam include beam current monitors (BCMs), beam position monitors (BPMs), wire array and synchrotron light monitor (SLM). The placement of the beam monitors at relatively low energy (1 GeV at ASSET) and at high energy ( $\sim 48 \mathrm{GeV}$ at A-Line) is indicated in Fig. 2.4.

\subsubsection{Beam Current Monitors}

The SLAC BCMs [41] are toroids made of copper wire wound around an iron core. When the electron beam passes through a toroid, it induces a pulse in the windings. The toroid acts as the inductive element of an RLC circuit that causes the induced pulse to ring. This ringing signal is amplified and then differentially transmitted almost 100 feet from the beam line to the readout electronics. The signal is then rectified by an absolute value circuit and fed into a custom 16-bit ADC (described later). 
To achieve the best possible signal to noise ratio, the $\mathrm{Q}$ value of the toroid RLC circuit is made as large as possible. This allows the toroid signal to be integrated for upwards of $1 \mathrm{~ms}$ in order to achieve high resolution in the measurement. However, with such a large $\mathrm{Q}$, if nothing is done to damp out the toroid charge, approximately one percent of the charge of one pulse will leak into the next pulse. This could both decrease the toroid resolution and create helicity systematics due to hysteresis. As the signal must be damped, the initial ringing circuit contains a transistor (used as a relay) which can connect a large resistor to the overall circuit. Approximately $3 \mathrm{~ms}$ into the pulse (after the integration is complete), this transistor is activated, damping out the pulse and assuring that no charge leaks into the following pulse. The trigger for the damping circuit is generated from the DAQ trigger.

Typical resolution on the toroid varies from device to device, but is usually around 60 ppm per pulse, which, when added in quadrature with counting statistics, increases the detector asymmetry distribution width by $\sim 8 \%$. The toroid linearity has also been measured with a calibrated charge pulse, and has been determined to be better than $99.9 \%$ [42].

Two pairs of BCMs are located a few meters upstream of the target. These toroids measure the beam's total charge and helicity correlated intensity asymmetry and are used to normalize the detected scattered flux in calculating the asymmetry.

\subsubsection{Beam Position Monitors}

The SLAC BPMs $[43,44]$ are resonant cavity monitors tuned to the accelerator's frequency of $2856 \mathrm{MHz}$. Each BPM has three cavities. Each cavity is tuned to resonate in a particular mode (TEM00, TEM10, or TEM01). When an electron 
pulse passes through the cavity, it excites the resonant mode with an amplitude proportional to the beam property to which that mode is sensitive. A TEM00 cavity is sensitive to the total beam current, a TEM10 cavity is sensitive to beam's horizontal displacement from the center, and a TEM01 cavity is sensitive to beam's vertical displacement from the center. An antenna inside the cavity picks up the induced signal, and the signal is transmitted almost 100 feet from the beam line to the processing electronics. The processing electronics mixes the signal with a reference $2856 \mathrm{MHz}$ oscillator locked to the accelerator's RF. The mixer outputs can be considered to be the "real" and "imaginary" parts of the BPM signal. During production running, the mixer's phase is offset for each BPM to maximize the real part of the signal and minimize the imaginary part. The real and imaginary parts are fed into 16-bit ADC's.

$\mathrm{BPM}$ linearity was measured by changing the attenuation on one BPM and comparing its signal to the closest BPM (each BPM is adjacent to or very near another, for redundancy and crosschecks). If the attenuation on one BPM is decreased, its overall dynamic measurement range will be reduced (this increases the overall resolution by increasing the signal to beam position ratio). For the data taken in Run I, all BPMs were run with dynamic ranges large enough to keep the BPMs more than $99 \%$ linear for nominal beam positions. In the analysis, a cut will be made to the data to eliminate instances in which the beam moved far enough off center to reduce any BPM's linearity below 99\%. Typical resolution on the BPMs varied from 1 to $3 \mu \mathrm{m}$ (see for example Fig. 2.5) during the course of the run (they usualy averaged around $2 \mu \mathrm{m}$ or less), for such reasons as phase drifts, cavity shape degradation, and dynamic range adjustments.

One pair of BPMs is located almost $2 \mathrm{~m}$ upstream of the target and measures beam position at the target. A second pair is located almost $40 \mathrm{~m}$ upstream of the 

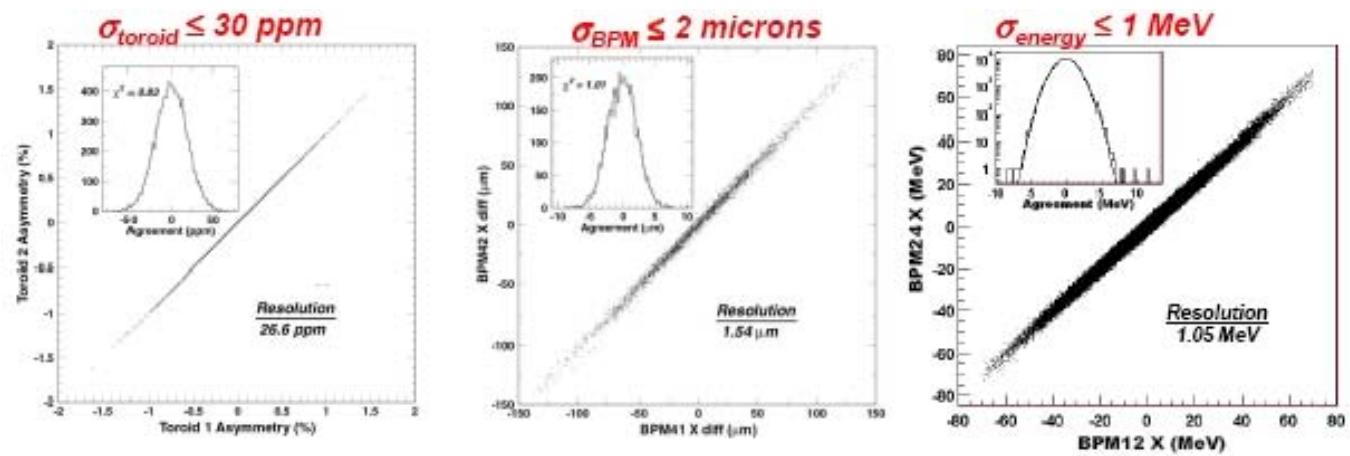

Figure 2.5: Resolution of different beam monitors.

target and provides information on the beam's angle at the target. These BPMs are used to remove, from the detector signal, correlations with beam position and angle. Another pair of BPMs is located at the middle of the A-Line bend, one before and one after the momentum defining slits. The beam position in the bend is very sensitive to variation in the beam energy from its nominal setpoint, so these two BPMs are monitors of the beam energy. These BPMs are used to remove, from the detector signal, correlations with beam energy.

\subsubsection{Synchrotron Light Monitor}

A synchrotron light monitor (SLM), located at the A-Line bend, measures the intensity of the synchrotron radiation emitted by beam. As the intensity of the synchrotron radiation is proportional to the beam energy, the SLM provides another measure of the beam energy. The power of the synchrotron radiation is proportional to $B^{2} E^{2}$, where $B$ is the magnetic field and $E$ is the beam energy. The measurement is therefore not linear in the energy, but is still useful as an independent measurement of the beam energy. 


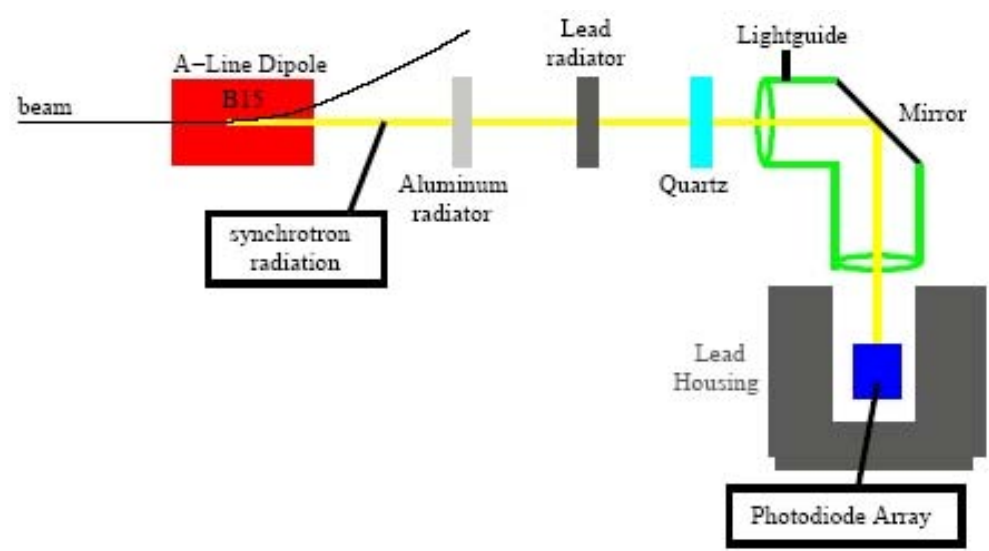

Figure 2.6: Schematic for the synchrotron light monitor.

To detect the synchrotron light, a lead radiator and a quartz Cherenkov radiator are used to downgrade synchrotron light at $1 \mathrm{MeV}$ into light in the visible spectrum [45]. The visible light is then run through a series of mirrors (Fig. 2.6) into a lead housing (shielded to prevent background from soft photon radiation) containing four photodiodes. The signal from these photodiodes is then fed into a standard 11 bit ADC.

\subsubsection{Wire Array}

A tightly packed array of $\mathrm{Cu}-\mathrm{Be}$ wires, located almost $1 \mathrm{~m}$ upstream of the target, provides a measure of the electron beam's intensity profile in two dimensions and is used to measure the beam spot size and other higher order moments. This wire array consists of two planes of wires, one running horizontally and the other vertically. The wires in each plane are 7 mil in diameter and have been placed 14 mil apart from one another. An aluminum foil is located next to the wires, and is raised to a high enough voltage to allow the beam to induce $40 \mathrm{mV}$ pulses on 

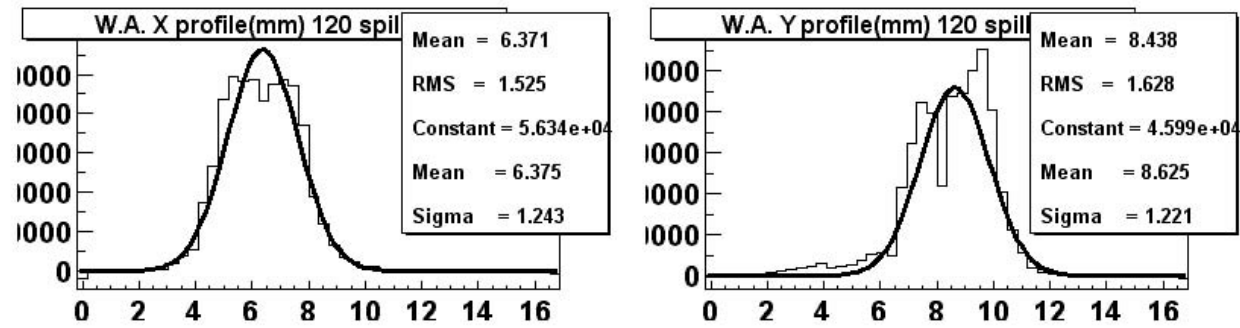

Figure 2.7: Typical output from the wire array, averaged over a one second period.

individual wires. This foil has a 1" hole so that the beam can pass through it. The resolution of the wire array is slightly better than $13 \mu \mathrm{m}$ in both axes. A typical output of the wire array is shown in Fig. 2.7.

\subsubsection{Beam Dithering Hardware}

At the beginning of the A-Line, there are several magnets which can be used for beam steering. These magnets have fast response time, and are used to intentionally change the beam's position and angle, in a controlled way, to calculate the correlation between the experimental asymmetry and the asymmetries measured in each beam monitor. A total of eight such magnets are used to move the beam: two redundant magnets each in $\mathrm{x}, \mathrm{y}, \mathrm{dx}$ and $\mathrm{dy}$. The modulation of the phase of a klystron near the end of the accelerator provides dithering of the beam energy.

\subsection{Liquid Hydrogen Target}

E158 uses a liquid hydrogen target as its source of target electrons. Liquid hydrogen is chosen for its high ratio of electrons to nucleons. This target contains the largest volume of liquid hydrogen ever used for a fixed target experiment. The target is 


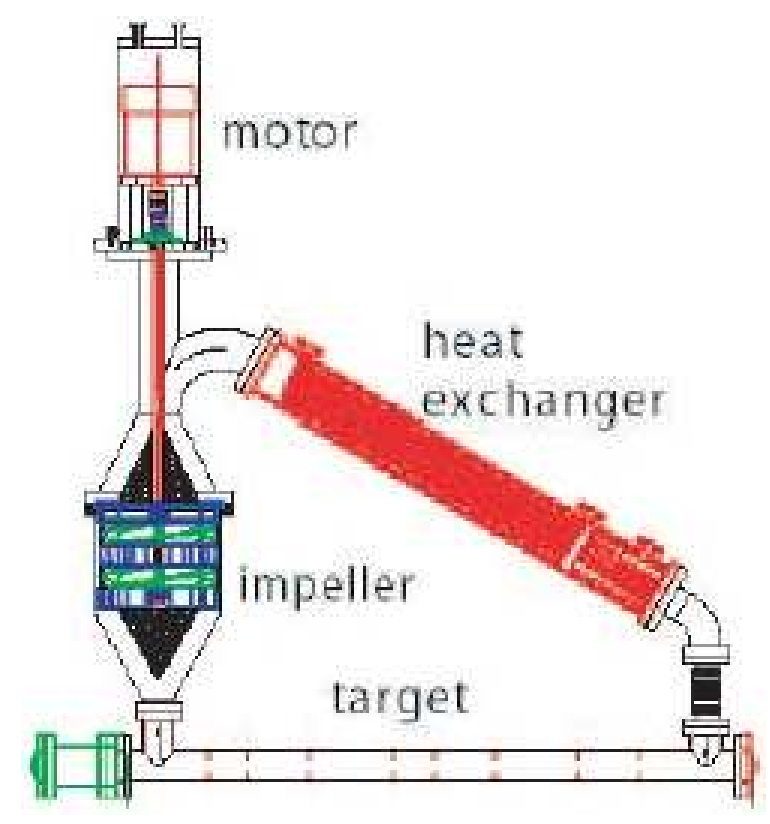

Figure 2.8: A schematic of the liquid hydrogen target loop.

insertable and removable from the beam line, and must survive sustained power of $700 \mathrm{~W}$ and a lifetime radiation dosage approaching $100 \mathrm{Mrad}$ [46]. Fig. 2.8 shows the schematic of the target.

The actual target is a $150 \mathrm{~cm}$ long aluminum cylinder (which corresponds to 0.15 r.l. of liquid hydrogen), 3 inches in diameter, filled with liquid hydrogen at an operating temperature of $17.5 \mathrm{~K}$. The cylinder is connected on one side to a heat exchanger and on the other side to a differential pump. The heat exchanger contains a copper coil through which helium (at $4 \mathrm{~K}$ ) flows, and is designed to remove up to $1000 \mathrm{~W}$ of heat from the target. The heat exchanger also includes a heater which is used to maintain the heat load on the target at a constant level when the beam current changes. The differential pump moves the hydrogen through the system at sustained flow rate of $10 \mathrm{~m} / \mathrm{s}$. This high velocity is necessary 

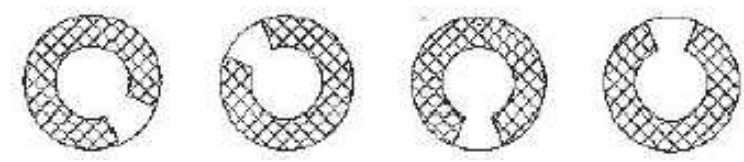

Figure 2.9: Examples of the wire mesh disks.

to prevent hydrogen density fluctuations (due to beam heating) from artificially increasing the width of the experimental asymmetry.

The target absorbs more than $500 \mathrm{~W}$ of power from the beam and yet is required to have pulse to pulse density fluctuations below the $10^{-4}$ level so as not to significantly degrade the statistical power of the measurement. The key design feature for suppressing density fluctuations is a series of eight wire mesh disks (Fig. 2.9) spaced along the target cell's length in order to introduce a transverse velocity component and generate turbulent flow at a size scale comparable to the beam diameter. Density fluctuations due to the induced turbulence were conservatively estimated to be below the $10^{-5}$ level [37]. Density fluctuations due to electron beam were studied by looking at the residual correlation between the Moller detector and luminosity monitor (described later) rates after removing all correlations with beam properties. An upper limit on density fluctuations during normal physics-running conditions $\left(120 \mathrm{~Hz}, 6 \times 10^{11}\right.$ electrons/pulse, $48 \mathrm{GeV}$, and $1 \mathrm{~mm}$ rms beam radius) at $65 \mathrm{ppm}$ was set [46].

The target loop is placed inside an aluminum scattering chamber, held at room temperature but evacuated to a pressure of roughly $10^{-8}-10^{-9}$ torr to prevent heat loss from convection. The loop is mounted so that it can be lifted out of the beam path remotely.

A LabView based DAQ monitors and controls the properties of the target. The DAQ displays a number of temperature and pressure measurements taken at 
several points around the loop and cooling lines and controls the power setting of the heater in order to keep the target temperature stable at the $0.1 \mathrm{~K}$ level.

\subsubsection{Moller Polarimeter and Carbon Targets}

To measure the electron beam polarization, a Moller polarimeter is installed just upstream the scattering chamber. The polarimeter contains supermendur (an alloy of $50 \%$ iron and $50 \%$ cobalt) foils of varying thicknesses $(20,50$ and $100 \mu \mathrm{m})$ that could be inserted into the beam line remotely. The foils are polarized by two custom designed Helmholtz coil magnets, placed perpendicular to the beam axis, which together produce a field of 92 gauss on the foil.

The scattering chamber also contains a table holding several carbon targets of various thicknesses that are used for spectrometer and detector studies. When the liquid hydrogen target is lifted clear of the beam, the table can slide horizontally to bring any one of the carbon targets into position. Interlocks ensure that only one of the targets - either the liquid hydrogen or one of the carbon targets - could be placed in the beam at one time.

\subsection{Spectrometer and Collimators}

The E158 spectrometer was designed specifically for this experiment, and is optimized for the detection of very forward angle $\left(0.27^{\circ}-0.41^{\circ}\right)$ Moller scattering with suppression of photon and $e P$ elastic and inelastic backgrounds. A schematic of the spectrometer is shown in Fig. 2.11. Fig. 2.10 shows an overview of the experimental setup in ESA. 


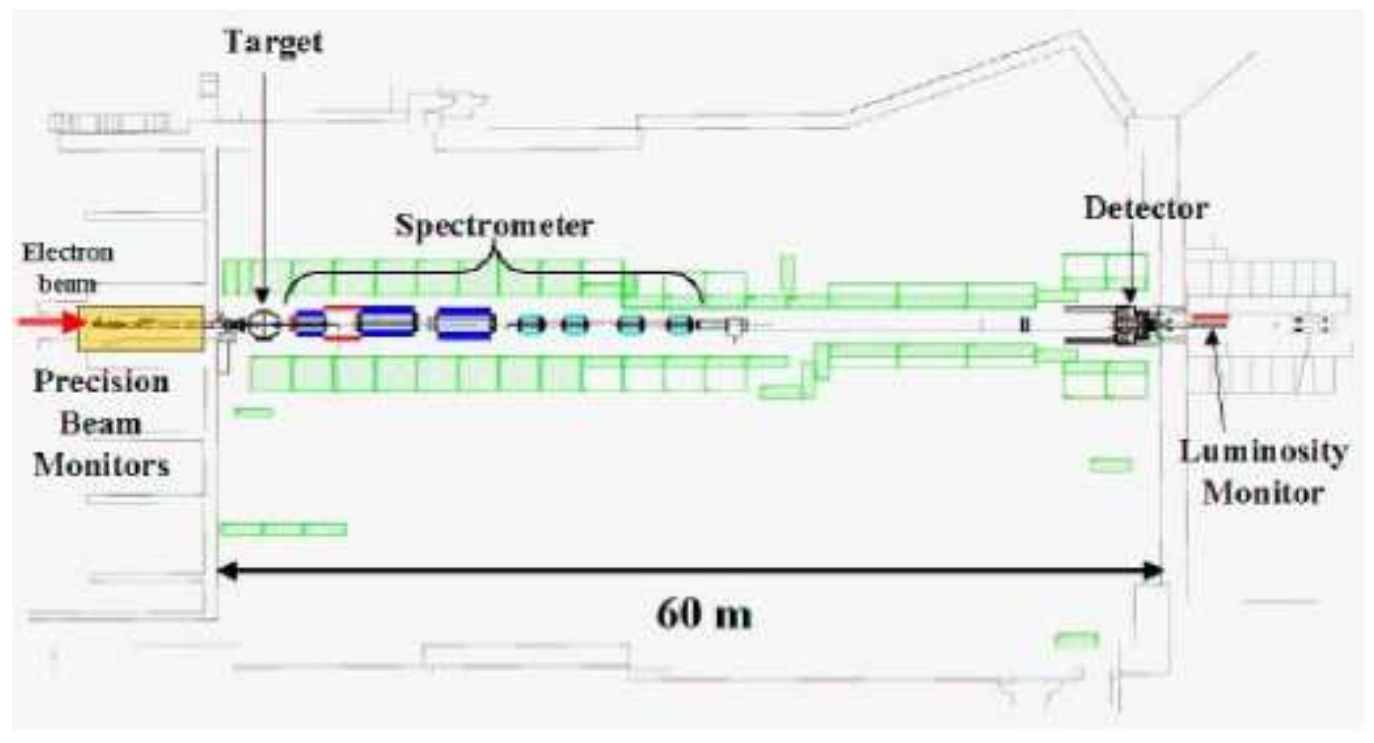

Figure 2.10: Overview of the experimental setup in End Station A.

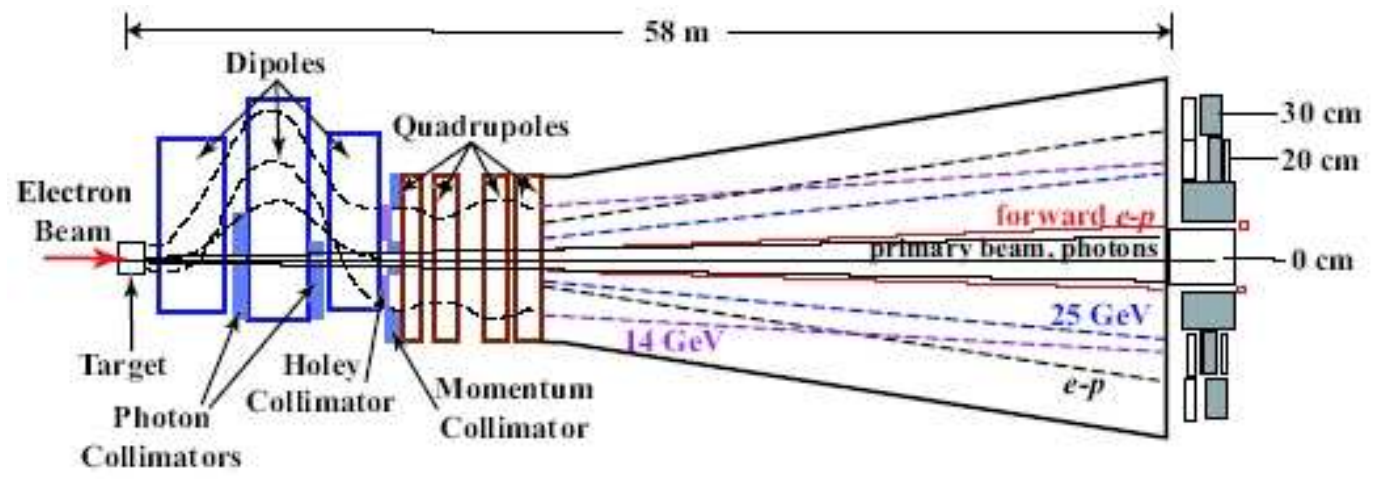

Figure 2.11: A top view schematic of the layout of the E158 spectrometer.

The spectrometer runs the entire length of ESA, almost 60 meters. A major constraint in designing the spectrometer is that no component that might see incident particle flux (and then radiate scattered particles into the detector region) can be made of iron. This is because the expected experimental asymmetry of around $150 \mathrm{ppb}$ is almost eight orders of magnitude smaller than the asymmetry 
from the polarized electron-polarized iron scattering. Almost all pieces of beam pipe are made from aluminum, and all collimators have been fabricated either from copper or from non-magnetic tungsten with a very low iron component.

\subsubsection{Dipole Chicane}

Aside from $e P$ scatters, the main potential detector background consists of soft (low momentum) particles created in the target. This flux contains photons, positrons, and electrons, including low momentum and low asymmetry Moller electrons. A dipole chicane is placed downstream of the target for suppression of soft backgrounds. This chicane, which uses three dipole magnets in $D \bar{D} D$ configuration, redirects the primary electron beam and allows collimation of the high-power photon beam generated by the target along the beam axis. The primary beam as well as the Moller electrons travel cleanly through the chicane. Each chicane magnet have fairly uniform field to preserve the azimuthal symmetry of the Moller signal profile. The azimuthal symmetry is slightly distorted leaving the chicane, but is corrected by the last quadrupole magnet (discussed below).

The placement of the dipoles is fixed by three constraints. First, the internal walls of every dipole need to be as far as possible from the high power photon beam to avoid significant amount of radiation damage. Second, the walls must not collimate any of the Moller flux. This limits the maximal beam bend angle in the chicane to $44 \mathrm{mrad}$ in the second dipole and half that in each of the other dipoles. The third constraint is that the three dipoles should together take as little room in $z$ as possible, thereby allowing the quadrupoles to sit as far upstream the detector as possible. 
The first dipole bends all electrons with momenta up to $5 \mathrm{GeV}$ into its inner left wall, and all positrons with momenta less than $28 \mathrm{GeV}$ into its inner right wall. The power in these fluxes is quite high. Consequently, two pieces of water cooled copper, each three inches thick, run the length of the dipole on either side. Similar water cooled masks have been placed in the second and third dipoles to absorb power due to synchrotron radiation and mis-steered electrons.

Additional copper masks have been placed between the first and second dipoles to block all electrons with momentum less that $9 \mathrm{GeV}$. The copper mask inside the first dipole does not stop at the end of the dipole, but rather continues downstream for another $29 \mathrm{~cm}$. A second copper mask starts a few more inches downstream of the end of this first mask, and runs downstream for $96 \mathrm{~cm}$. A third piece of copper, located immediately upstream the second dipole collimates all electrons with momenta less than $9 \mathrm{GeV}$. Each of these copper pieces is water cooled. Since, the electrons strike the third piece of copper head-on, it has been made 40 r.l. thick to prevent punch through.

The primary beam's energy is left mostly unaffected by the hydrogen target; thus, primary beam electrons pass through the dipoles with momenta around $45 \mathrm{GeV}$. The Moller electrons, however, scatter from the target at energies from 11 to $24 \mathrm{GeV}$. All charged particles undergo synchrotron losses in the chicane, with the amount of energy loss proportional to the square of the particle momentum. As a result of the different changes in the momenta of beam and Moller electrons, the net magnetic force on the Moller signal is slightly different than it is on the beam particles. This makes the Moller signal profile to shift (towards left) about three $\mathrm{mm}$ off-center at the detector face. The field of the third dipole is adjusted accordingly to keep the Moller signal profile centered on the detector. 


\subsubsection{Photon Collimator}

Once the primary beam has been deflected away from the beam axis by the chicane, the target photons which have scattered at wide angles can be collimated. For this purpose, two cylindrical "Photon" collimators are placed on the beam axis, which block the line of sight between the target and the Moller detector and the luminosity monitor. Tungsten is used for the collimators in order to create a "hard edge" to the collimation, minimizing the number of particles which might shower off the inner surface and travel downstream to the detector. To minimize "punchthrough" leakage, each cylinder is made 40 r.l. thick. One collimator is located between the first two dipoles (Fig. 2.12) and the other at the downstream end of the second dipole.

Even with both photon collimators, the entire line of sight between the target and the detector is not blocked - a small gap in coverage exists on the left side of

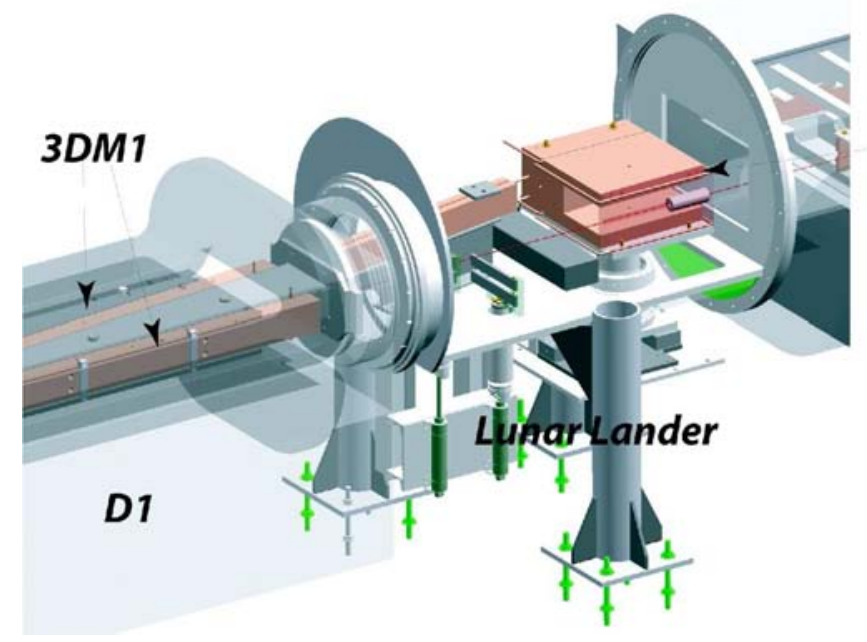

3DC2C

photon collimator (soft shadow)

Figure 2.12: Cad diagram of the assembly "3DC2C" between the first two dipoles that contains the first photon collimator. 
the beam axis. Two pieces of copper have been placed in the second dipole to block photons in this region. A small groove cut into both pieces prevents synchrotron radiations from striking (and melting) the uncooled copper.

\subsubsection{Momentum Collimator}

The momentum collimator or the radial collimator sits immediately downstream of the third dipole. This collimator defines the momentum acceptance of the spectrometer. It is made of two concentric cylinders, connected by two spokes in the horizontal plane as shown in Fig. 2.13. It passes Moller electrons with momenta in the range 13-25 GeV and $e P$ electrons with momenta of $\sim 40 \mathrm{GeV}$. Fig. 2.13 also shows a simulated profile of the Moller and $e P$ fluxes as a function of distance from the beam axis. Quadrupole magnets downstream of the collimator then separate the Moller and $e P$ fluxes.
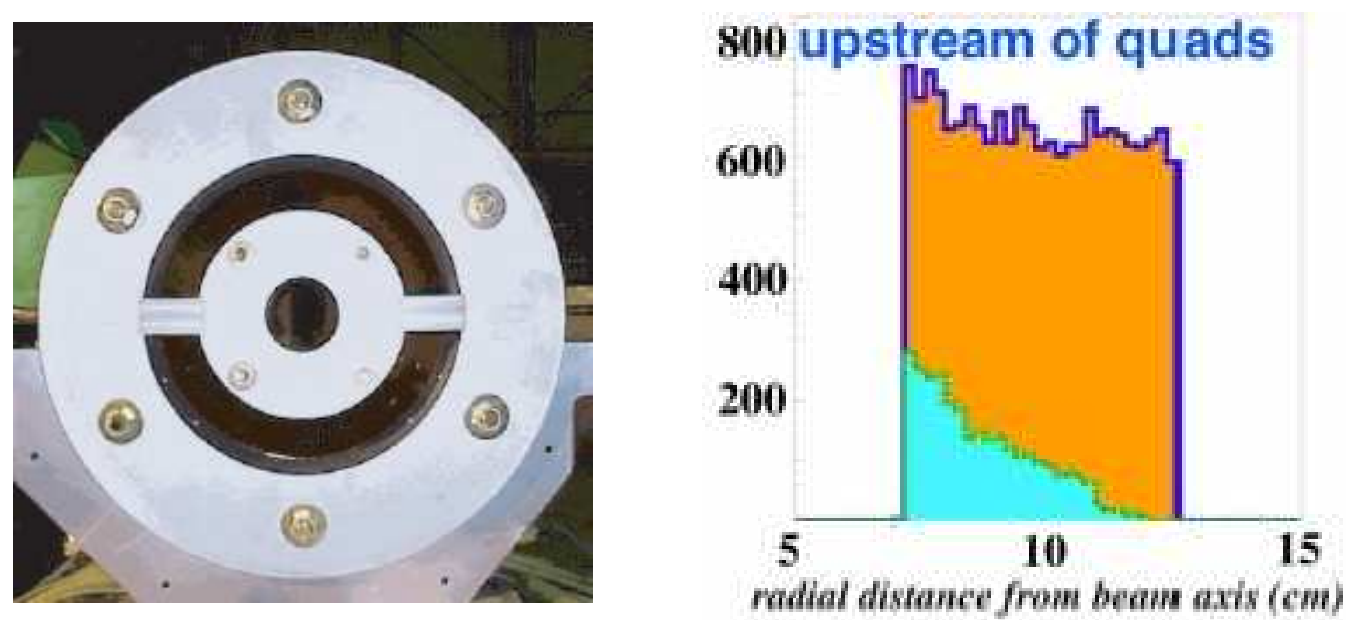

Figure 2.13: (left) Momentum collimator, (right) Moller and eP scattered flux at the acceptance defining collimator. 
The momentum collimator actually consists of two pieces - an upstream copper collimator and a downstream tungsten collimator - which have been brazed together to create a single piece. The overall thickness of the piece is 40 r.l. (roughly equally divided between tungsten and copper), again to prevent punch-through. The entire collimator is water cooled.

\subsubsection{Synchrotron Collimators}

The inner and the outer cylinders of the momentum collimator are connected by two sizable pieces of metal. These "spokes" block the broad swath of synchrotron radiation created in the chicane in the horizontal plane. Each spoke is 40 r.l. thick. If the electron beam has a transverse polarization component, it can couple to misalignment of the dipole magnets and induce a helicity correlated asymmetry in the intensity of the synchrotron radiation. The synchrotron collimators are designed to suppress the synchrotron radiation background at the detector face by a factor of 100 .

The spokes in the momentum collimator cannot collimate all the synchrotron radiation, since a sizable fraction of it passes through the center of the collimator. To block this background, two more sets of collimators are placed at downstream locations. The first set is located immediately after the fourth quadrupole magnet. These collimators are made of tungsten, water cooled, and each is 20 r.l. thick, enough to completely stop the MeV photons. The second set of synchrotron collimators are bolted directly on the detector face. These collimators are also made of 20 r.l. tungsten but require no water cooling. Both sets of these downstream spokes are located in the shadow of the momentum collimator. Eleven percent of the Moller flux is lost due to the synchrotron collimators. 


\subsection{5 "Holey" Collimator}

Immediately in front of the momentum collimator sits a remotely insertable "Holey" collimator (Fig. 2.14), which is essentially a mirror image of the momentum collimator. It consists of two semi-circles designed to entirely cover the Moller signal acceptance region of the momentum collimator except for four $1 \mathrm{~cm}^{2}$ holes cut into this collimator at various azimuthal angles and radii. These holes are all $90^{\circ}$ apart from one another, and are placed to allow a very precise measurement of the elastic $e P$ signal. When this collimator is inserted, each hole allows only a small momentum bite to reach the detector plane, resulting in excellent separation of the Moller and $e P$ elastic peaks. The remaining flux between the peaks is due to inelastic $e P$ scattering. These measurements of the inelastic $e P$ flux as a function of radius are necessary to apply an accurate background correction.

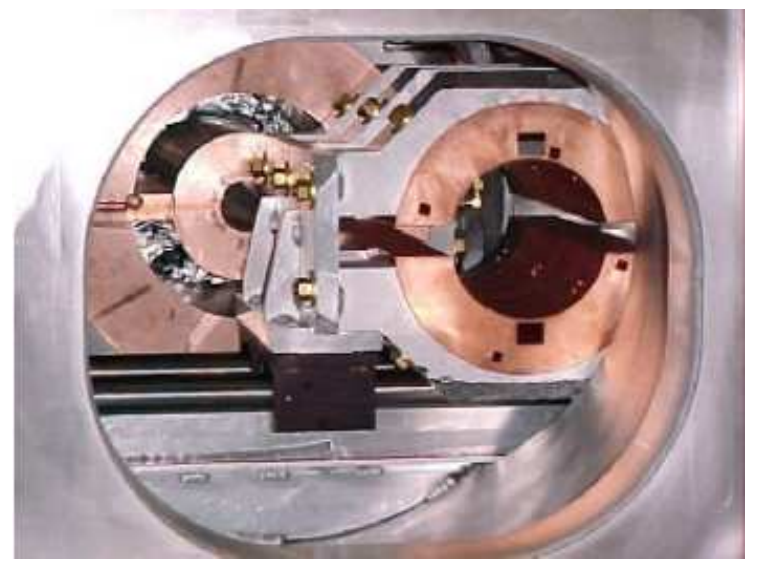

Figure 2.14: A photograph of the momentum collimator and the holey collimator in their segment of beam pipe.

Two more holes are cut into the holey collimator for the purpose of polarimetry. Measuring the beam polarization does not require a full azimuthal acceptance, but does require a smaller radial acceptance than allowed by the radial collimator. 
Therefore, these two holes are $2 \mathrm{~cm}$ high and $2.6 \mathrm{~cm}$ wide, and are centered on the vertical axis.

\subsubsection{Quadrupole Magnets}

Four quadrupole magnets are used to separate Moller and $e P$ fluxes. These quadrupole magnets are located immediately after the momentum collimator in order to maximize the drift distance to the detector. Each quadrupole has a field gradient that is uniform to $0.1 \%$ in the entire region where Moller electrons will travel [16]. The magnet positions and strengths are optimized to simultaneously maximize the separation between the Moller and $e P$ fluxes and maintain the azimuthal asymmetry of the fluxes. The quadrupoles' focusing is proportional to the

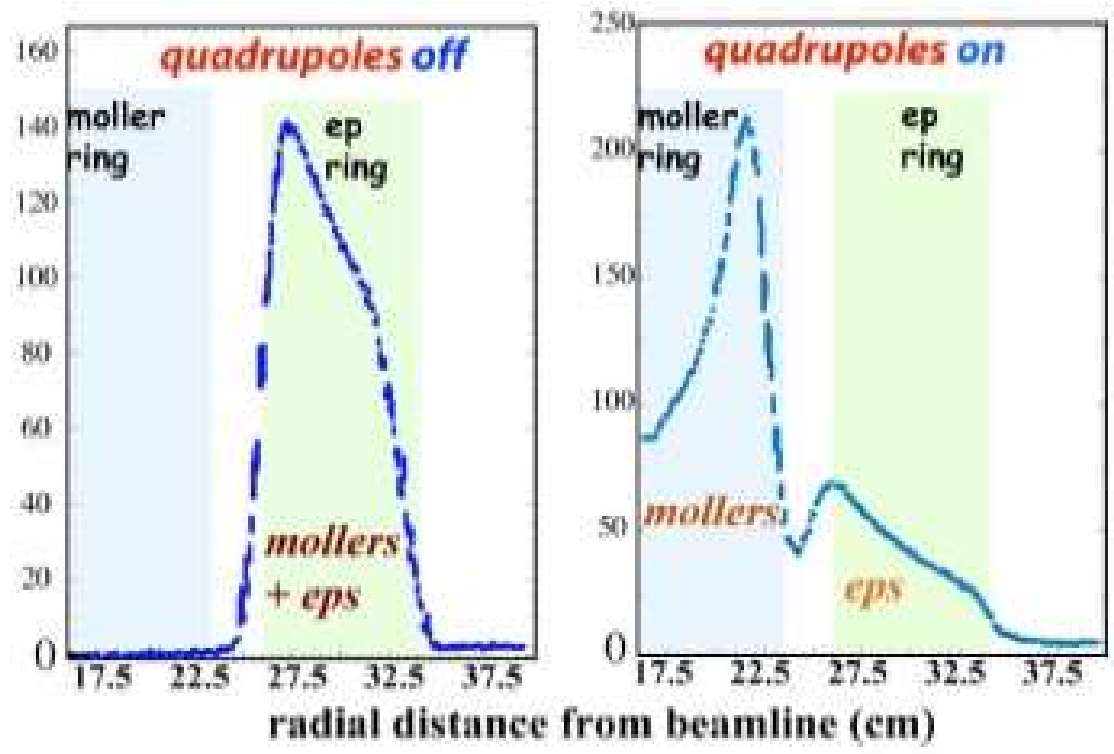

Figure 2.15: Measured electron profile at the detector plane with quadrupole magnets off (a) and on (b). The blue and green band indicate the radial acceptance of the detectors for Moller and $e P$ electrons, respectively. 
energy of the particles passing through them, and so the lower energy Moller flux is much more strongly focused than the eP flux. Fig. 2.15 shows measurements of the radial profile of the flux at the detector plane for the quadrupole magnets off (a) and on (b). With the quadrupole magnets off, a single peak that includes both Moller and $e P$ electrons is visible, centered on a radius of $\sim 30 \mathrm{~cm}$. This profile looks like the sum of the Moller and $e P$ profiles in Fig. 2.13, allowed to drift to the detector plane. Fig. 2.15(b) shows the profile with the quadrupoles on: two peaks are now visible, with the inner peak being predominantly the Moller scattered electrons and the outer peak the $e P$ electrons, respectively.

\subsubsection{Drift Pipe}

To prevent signal degradation in the air between quadrupole four and the detector face $30 \mathrm{~m}$ away, a very large aluminum pipe is used to maintain a decent vacuum in the volume (Fig. 2.16). The synchrotron collimators downstream of the fourth quadrupole are located in this pipe, as well as the collimator masks described below. An aluminum conical flange connects this pipe with detector beam pipe. This cone is 0.375 " thick, which allows signal particles to pass through and onto the detector face without significant degradation.

\subsubsection{Collimator Masks}

A large amount of soft and hard photon background comes from the edges of the collimators, especially the second photon collimator and the momentum collimator. Seven "collimator masks" are installed to block the line of sight between the inner edges of these collimators and the detector face (Fig. 2.16). These collimators masks consists of tungsten rings supported by horizontal tungsten spokes thin 


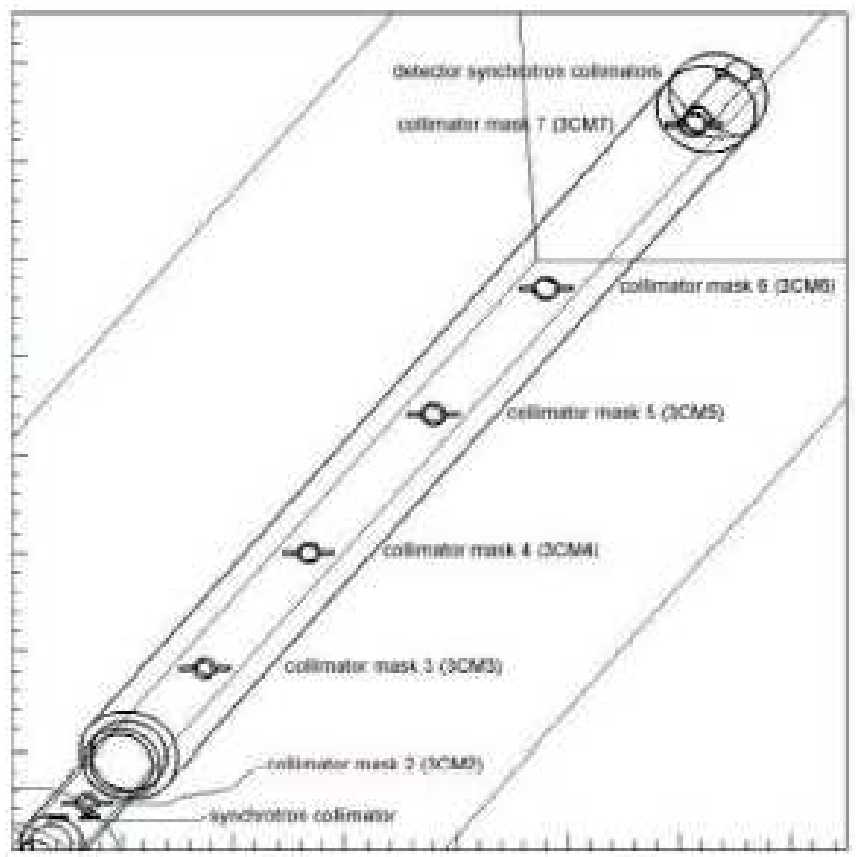

Figure 2.16: Schematic of the drift pipe, showing relative positions of the collimator masks as well as the synchrotron collimators.

enough vertically to sit in the shadow of the synchrotron collimators, yet thick enough in $z$ to completely block any incident synchrotron radiation or collimator shower products. The first and the last of these rings are water cooled.

The outer edges of these collimators are constrained by an imaginary line drawn between the inner (radial) edge of the Moller flux at the end of the fourth quadrupole, and the inner edge of the Moller detector. Although, this line seems safe, each piece nevertheless sees incident Moller and $e P$ flux, since the quadrupoles over focus a small fraction of Moller electrons that pass through the momentum collimator. Even so, if these particles shower off a collimator mask and into the detector, the overall detector resolution should not be affected, since the fluctuation in these particles should be equivalent to fluctuations in the signal electrons. 


\subsection{Detectors}

E158 uses a number of detectors to make both the physics measurement as well as several necessary auxiliary measurements. These detectors include the Moller detector, the $e P$ detector, the pion detector, the profile detectors, the polarimetry detector, and the luminosity monitor. An overhead view of most of the detectors can be seen in Fig. 2.18. The Moller detector, which is the main detector for the physics measurement, and the $e P$ detector will be discussed in detail separately in the next chapter. This section describes all the auxiliary detectors.

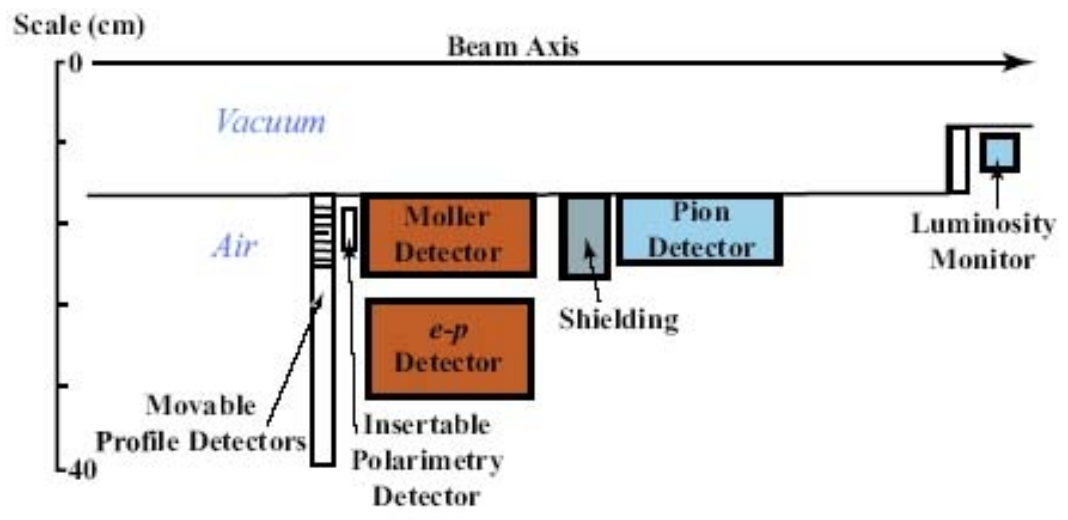

Figure 2.17: Schematic of the E158 detector package.

\subsubsection{Profile Detector}

The profile detectors are used to map the radial and azimuthal flux distribution incident on the Moller and $e P$ detectors. They consist of four Cerenkov counters mounted on a large wheel capable of rotating 180 degrees (Fig. 2.19) in order to cover the full range in azimuth. Each detector consists of a piece of quartz, a vacuum light guide, and a PMT to detect the Cerenkov light. The counters 


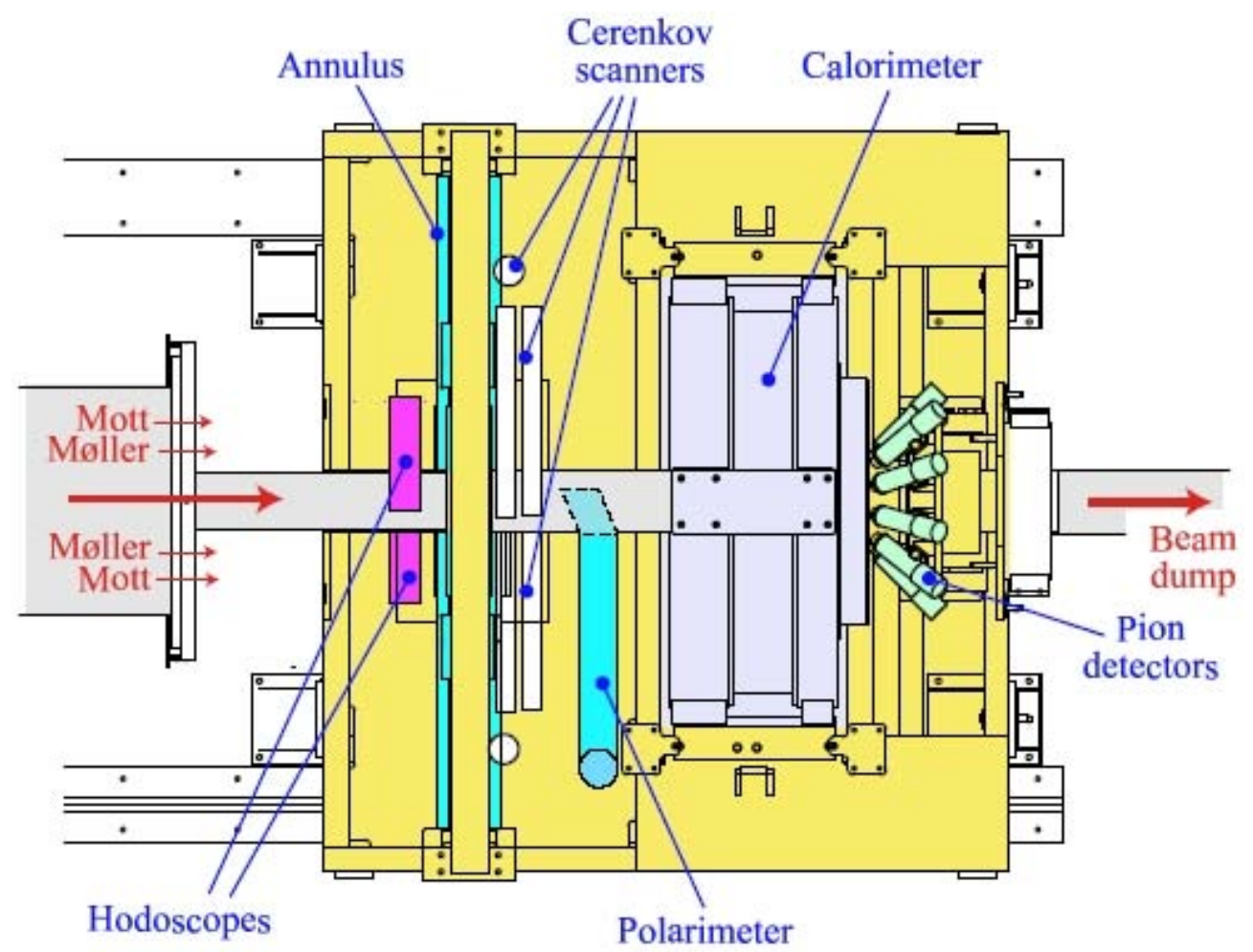

Figure 2.18: Overhead view of the Moller detector ("Calorimeter"), the pion detector, the profile detector ("Annulus" with "Cerenkov scanners"), the polarimeter.

are each mounted on an "arm" capable of moving the assembly radially inward (to $15 \mathrm{~cm}$, the closest it can be without striking the beam pipe) and outward (to $55 \mathrm{~cm}$, so that it doesn't block the Moller flux during the normal running). The motion of the wheel and each counter is controlled via LabView. The profile detector wheel is located just upstream of the Moller detector.

Large amounts of soft background are present around the wheel. This background originates from the drift pipe and the aluminum cone in from of the detector, both of which are hit by energetic electrons. Two of the Cerenkov counter assemblies contain features designed to minimize this background as shown in 

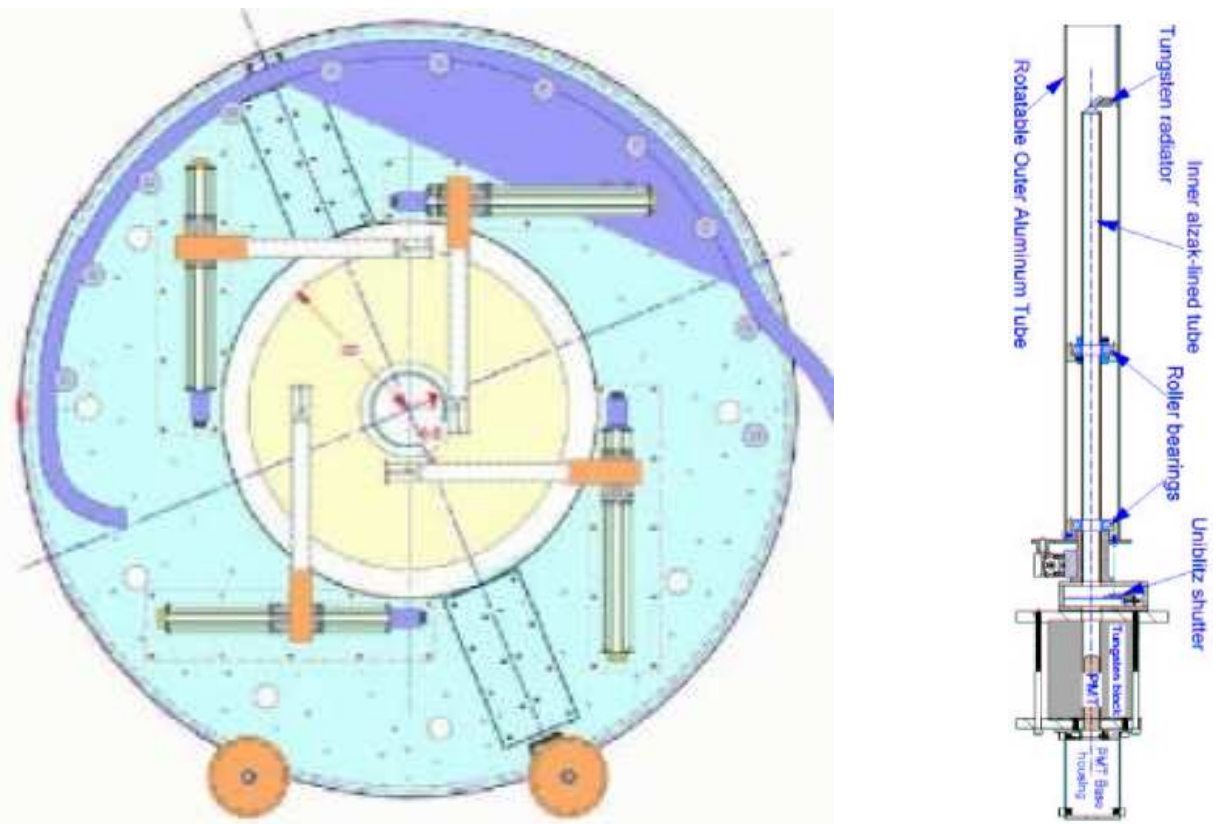

Figure 2.19: (left) Schematic of the Profile detector wheel, showing four Cerenkov counters sitting on their movable drives, (right) Single Cerenkov counter assembly.

Fig. 2.19. First, a remotely insertable/removable tungsten preradiator can be placed in front of the quartz, blocking low momentum particles and allowing the high momentum particles to shower before hitting the quartz. Second, an insertable/removable shutter in the counter assembly can block all photons from the quartz, allowing a clean measurement of the signal originating within the PMT. Both additions allow the profile detector to make a very precise measurement of the Moller and $e P$ fluxes.

\subsubsection{Pion Detector}

The pion detector is used to measure the pion flux and asymmetry. It is located immediately behind the Moller and $e P$ detectors (Fig. 2.18), and is well shielded by 
them from the Moller and $e P$ signals and photon backgrounds. However, high energy pions are able to punch through the Moller calorimeter and the lead shielding behind the calorimeter to reach the pion detector. The pion detector consists of 10 PMTs, each of which receives Cerenkov light from an adjoining quartz block [47]. The ten phototubes are equally spaced azimuthally; each phototube/quartz assembly is placed at $45^{\circ}$ from the $z$ axis. Since the pion detector signals are expected to fluctuate on the order of $0.1 \%$, the electronics for the detector are simple - each PMT signal is fed to 16-bit ADCs similar to those used for the BPMs.

\subsubsection{Polarimeter}

To measure the polarization of the beam, polarized electrons are scattered off a longitudinally polarized supermendure iron foil target described earlier. The holey

Figure 2.20: A diagram of the polarimeter. The lead shielding in front of the polarimeter is not visible.

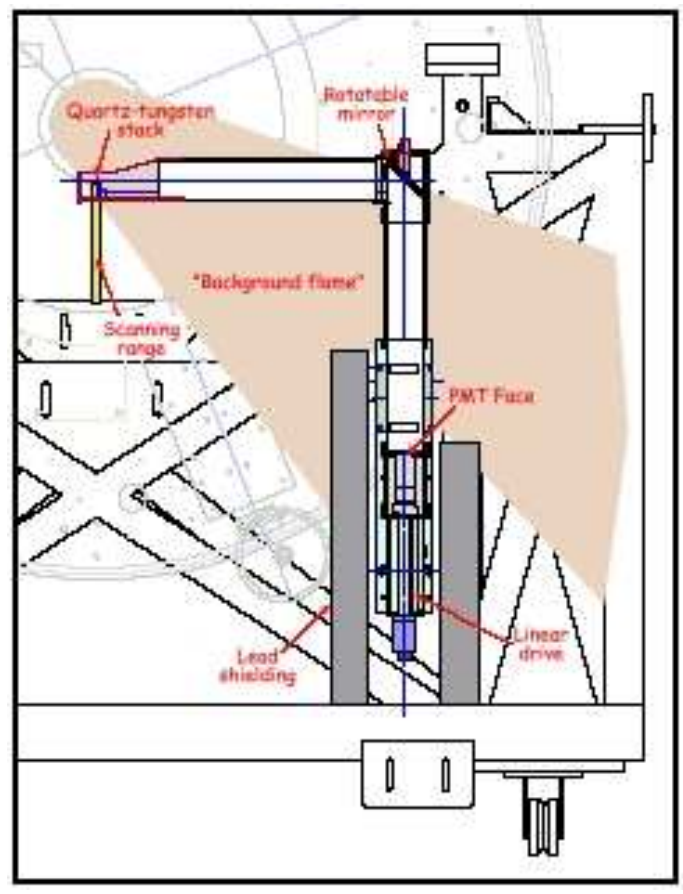


collimator is used for this measurement to allow only a small bin in radius and azimuth to reach the detector plane. Furthermore, a different setting is used for the spectrometer quadrupoles. Both changes help separate the Moller electrons from the $e P$ background. An additional small Cerenkov calorimeter (the "polarimeter", Fig. 2.20), which is located between the profile detector and the Moller detector, is used to detect this Moller signal. The polarimeter is made of alternating pieces of tungsten (seven) and quartz (six) [48]. Each plate is tilted at a $30^{\circ}$ angle from the vertical, and the size of the pieces have been optimized to measure Moller scatters which pass through the lower hole of the holey collimator while minimizing the background. The tungsten plates contain reflective surfaces designed to maximize light collection. Light from the quartz is fed into a highly reflective light guide. The light runs horizontally through the guide, gets reflected off a mirror, and then travels vertically to the PMT. The mirror can be rotated to block all light from the tungsten/quartz assembly so that the amount of background directly hitting the PMT can be estimated. The output of the PMT is fed to an 11-bit ADC. The entire light guide/PMT assembly is shielded behind six inches of lead. The polarimeter is remotely inserted during the polarization measurement only, and is parked away from the beam line $(\sim 50 \mathrm{~cm})$ during normal physics running.

\subsubsection{Luminosity Monitor}

The final detector used in the experiment is a luminosity monitor, located 7 meters downstream of the Moller detector. This monitor detects extremely forward angle $\left(\sim 0.1^{\circ}\right)$ Moller and $e P$ electrons, and is useful for two reasons: First, no asymmetry is expected in the very forward scattered Moller and $e P$ electrons, so the luminosity monitor should measure a null asymmetry (after having been nor- 
malized by the toroid charge), verifying the lack of a systematic asymmetry in the physics results. Second, once the fluctuations in beam parameters have been removed from the luminosity monitor and Moller detector signals, there should be no correlation between the signals. Any, residual correlation can indicate the presence of target density fluctuations.

The luminosity monitor consists of two separate detectors. Each detector consists of eight trapezoid shape gas chamber proportional ion counters, arranged in a ring around the beam pipe (Fig. 2.21). Every chamber contains eleven parallel aluminum plates, alternately kept at either 100 or 0 volt to maximize the signal amplification without causing extraneous arcing in the chamber. In addition, every gas chamber is filled with nitrogen. In front of each detector ring sits a ring of aluminum, used both as a preradiator and as a shield against synchrotron radiation. The signals from the plates are read in differential mode, and are fed into the ADCs similar to the BPM ADCs. The two detectors are located only a few inches apart to give a level of redundancy to the measurement.

Figure 2.21: Schematic of the front view of the luminosity monitor.

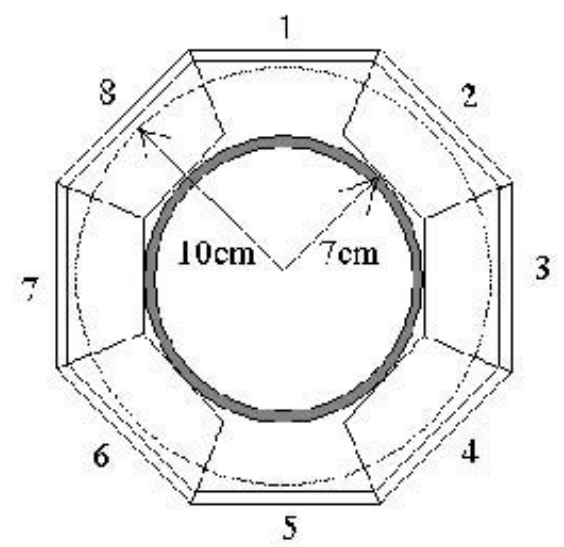




\subsection{Data Aquisition System}

As mentioned earlier, the experiment employs a flux counting technique; the response of the calorimeter is integrated over the duration of each pulse, and helicity asymmetry is determined from this. The main component of the readout electronics is analog to digital converters (ADCs), which are used to readout the scattered flux as well as beam position monitors and toroids to characterize each beam pulse.

The data acquisition system (DAQ) is run at $120 \mathrm{~Hz}$, and collects approximately $2 \mathrm{kB}$ per pulse. It is responsible to acquire data from several systems: the detector readout electronics, the A-Line beam diagnostics readout electronics, the ASSET beam diagnostics readout electronics, and certain polarized source laser diagnostics readout electronics at the injector. The DAQ also controls the beam asymmetry feedback hardware at the injector. A block diagram of the DAQ system and electron beam control is shown in Fig. 2.22. The electron beam parameters at the polarized source are controlled through the SLAC Control Program of the Main Control Center (MCC SCP) from ESA. The control of the beam polarization is through the PMON system (described earlier), which transmits the beam helicity, Pockels cell modes, Pockels cell voltages, half wave plate status, and an error bit to the ESA DAQ on each beam pulse.

\subsubsection{Integrating ADCs}

The integrated response of the calorimeter must be measured with a relative precision of $2 \times 10^{-4}$ over the duration of each beam pulse. However, the fluctuations in the beam parameters could degrade this uncertainty significantly. One must, therefore, measure the physical characteristics of the beam, such as energy, position and angle, with a relative precision better than $5 \times 10^{-5}$. 


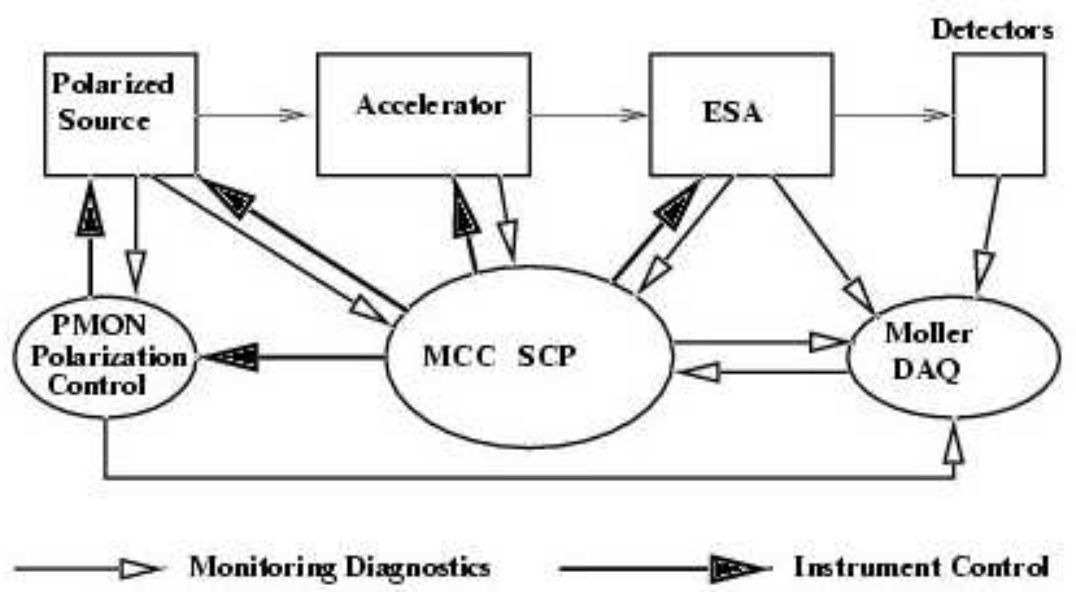

Figure 2.22: Schematic of DAQ system.

The detector and the beam diagnostics that require high resolution of readout electronics are fed into ADCs with true 16-bit resolution, designed at SLAC specifically to meet E158 requirements. The ADCs are required to have integral nonlinearity at the level of $0.1 \%$ and differental nonlinearity at the level of 1 least significant bit [4].

Each ADC board contains six channels. The channels can be run in either single-ended or differential mode. The differential mode halfs the maximum possible input but allows the integration of positive and negative signals. Each channel contains a gain stage, an integrator, and a digital sampling chip. The integrator gain, the timing of integration, and several other parameters are all remotely programmable. Each ADC receives its trigger from a master board, which in turn receives trigger from the main data acquisition system. The ADCs integrate the input signal by charging a capacitor, measure an analog difference between the voltages across the capacitor before and after integrating the signal pulse, and digitize that difference with 16-bin resolution. Same ADC boards are used for the 
Moller and $e P$ detectors, the luminosity monitor, the pion detector, the BPMs and the toroids. The values of the integration capacitor and the resistors which set each channel's gain are optimized for each type of measurement.

\subsection{Helicity Correlated Feedbacks}

As discussed earlier, the sign of the beam helicity is changed pulse by pulse by controlling the handedness of the circularly polarized laser light incident on the photocathode of the polarized source. The laser circular polarization is in turn controlled by changing the polarity of the high voltage applied to the Pockels cell in the laser transport system. Most of the false asymmetries due to change in the beam characteristics with helicity reversal can be traced back to helicity correlations of the intensity and position at the polarized source.

Obtaining negligible helicity correlations is not automatic; even a carefully prepared optical system can give rise to, for example, laser intensity asymmetries at the level of $100 \mathrm{ppm}$. These asymmetries are maintained at a negligible level using three active feedback loops. One feedback loop balances the intensity asymmetry between the two helicity states. This is accomplished by controlling the laser intensity asymmetry with a Pockels cell (IA) immediately upstream the CP and PS cells (Fig. 2.3). This cell can be pulsed at $120 \mathrm{~Hz}$ with a helicity-correlated voltage, introducing a phase shift into the beam. The downstream CP Pockels cell then turns this phase shift into a helicity correlated intensity, negating any measured beam intensity asymmetry.

A second feedback loop negates position asymmetries in the laser beam. At one point, the laser reflects off a mirror (called "piezomirror") mounted on several piezo-electric crystals. These crystals expand or contract under high voltage, and 
can be pulsed at $120 \mathrm{~Hz}$ to translate the mirror up to $6 \mu \mathrm{m}$ at any one point. This produces helicity-correlated displacements of the laser beam on the photocathode up to $50 \mu \mathrm{m}$ [37], negating position asymmetries.

The third feedback loop provides a mechanism for keeping the corrections induced by the IA loop small. It looks at the correction induced by the IA loop averaged over a specified length of time and adjusts the CP and PS cell voltages in such a way as to drive IA loop correction to zero. Essentially, it compensates for the drifts in the polarization state of the laser beam that can give rise to an intensity asymmetry.

The intensity and position feedback loops utilize measurements of the beam parameters from low energy beam diagnostics at ASSET. Additional independent diagnostics at both low and high energy are used to monitor performance of the feedback loops and to measure beam asymmetries at the target.

All optics used to make the asymmetry feedback are located at the source. Although an ideal feedback system could remove all measured beam asymmetries, it not possible for the source optics to remove any beam energy asymmetry. However, the beam energy asymmetry is mostly proportional to the beam intensity asymmetry, so the intensity feedback should take care of the energy asymmetry.

The feedbacks on beam asymmetries are implemented by a program called FbAnal. FbAnal analyzes all the data in real time and tags each event as to whether or not it is used in the feedback analysis. It calculates the asymmetries from the data in real time and then sends signals through the DAQ up to the source to make appropriate changes in the voltages on the source pockels cells and piezomirror to nullify the beam asymmetries. 


\subsection{Online Monitoring}

Several of the experiment's detectors are monitored in real time to assure proper functioning of the apparatus. The real time analysis software takes data from DAQ and writes "memory mapped" files on the computer it is run. These map files are created using the $\mathrm{ROOT}^{1}$ classes and are updated every two seconds. The map files contain the "stripcharts", which are time histories of the detectors' and monitors' channels, and contain the data representing the channels' outputs for last two minuets. The data include the output values of each channel, averaged over 120 spills, and the rms.

A second software, which is run on the same computer where the map files are created, is used to view the stripcharts. This software employs ROOT GUI classes to create a user friendly graphical user interface (GUI), containing several pulldown menus. It reads the map files and plots the stripcharts for the channels selected from the pulldown menus. Other than the stripcharts, the software also plots the current channel values verses the detector channel for the Moller, $e P$ and pion detectors and the luminosity monitor. These charts are also updated every two seconds. For typical running, a few charts are continuously monitored. Fig. 2.12 shows examples of different plots created by the monitor software.

A real time alarm system also exists within the monitor software. This feature simply compares the current values and rms of all the channels with the "limits". The limits can be set, and saved, using the "dialogue boxes" created using the ROOT GUI classes. If the current value of some channel goes out of limit, for a set length of time, the monitor software produces a small beep and pops up the stripchart of that particular channel on the screen to inform the user. The

\footnotetext{
${ }^{1}$ ROOT is CERNLIB's data analysis software.
} 
Fig. 2.12 also shows an example of the dialogue box used to set the limits.

Figure 2.23: Different screen shots of the online monitoring software.
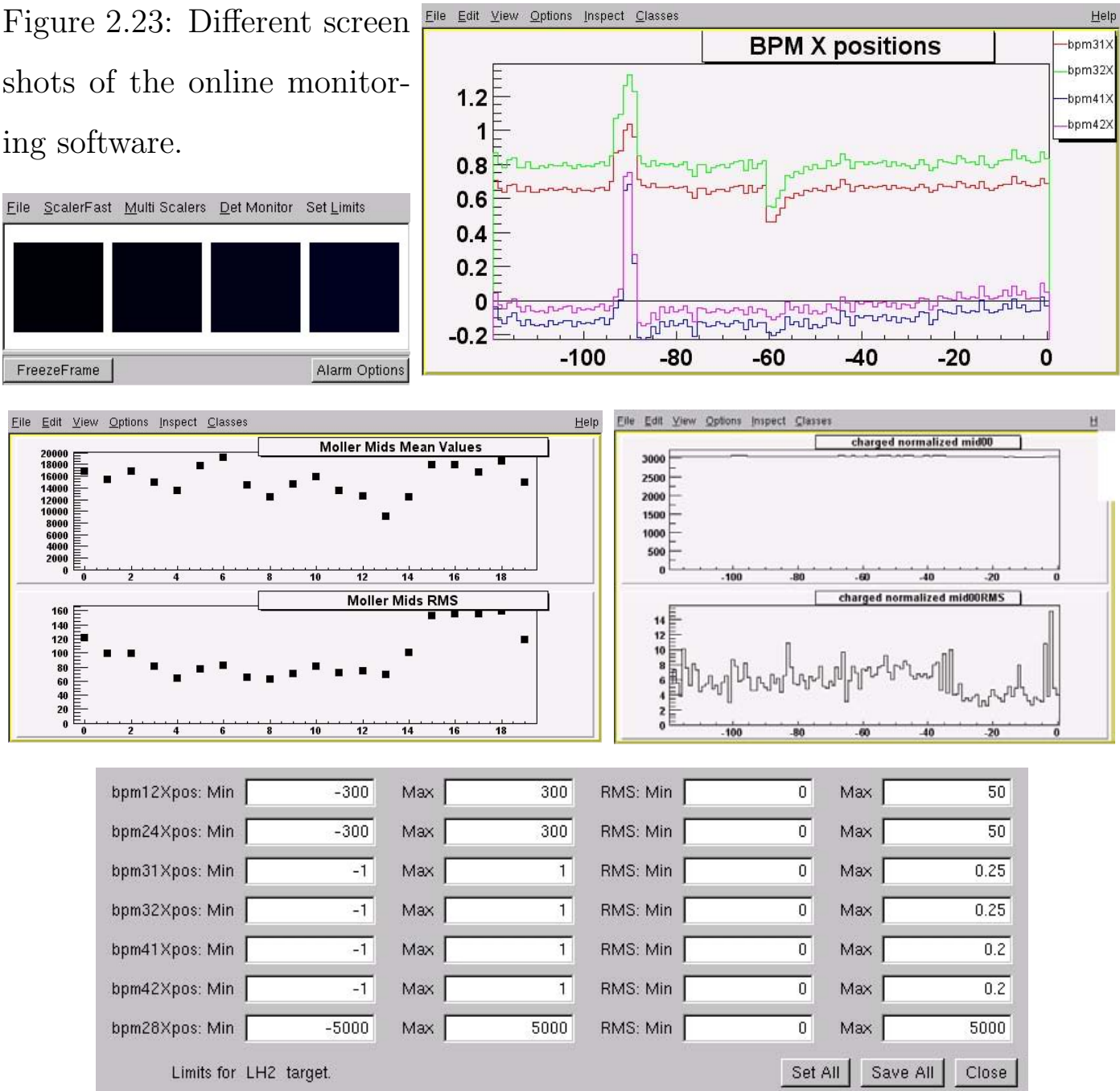


\section{Chapter 3}

\section{E158 Calorimeter}

\subsection{Moller Detector}

The Moller electron calorimeter is the primary detector in the experiment. The design is driven mainly by the need to integrate the calorimeter response to determine the scattered flux over the duration of the beam pulse. Basic requirements for the detector are:

- Maximum response to elastically scattered electrons.

- Moderate energy resolution $\sigma_{E} / E \approx 10 \%$ in the range from $10-25 \mathrm{GeV}$.

- Small response from pions, and from low energy photons and hadrons.

- No response from nuclear breakup or heavy ions (non-relativistic particles).

- Excellent radiation resistance.

These requirements are met by using the technique of "Quartz fiber Calorimetry" (QCal) which has been extensively studied for very forward calorimeters (VF- 
Cal) $[49,50]$ for the LHC experiments. The term "quartz" is a misnomer because it refers to a crystal whereas the fibers used in QCal are made of amorphous silica $\left(\mathrm{SiO}_{2}\right)$.

The basic principle for quartz fiber calorimetry is rather simple: the charged particles from a shower generated in a dense, high-Z absorber produce Cherenkov light in quartz optical fibers interspersed in the absorber. The same fibers act as optical guides for the generated light which propagates towards the photondetector.

The nature of Cherenkov light gives rise to several important benefits. Since the Cherenkov radiation is intrinsically a very fast process with a typical time constant of less than $1 \mathrm{~ns}$, it makes the quartz calorimeter intrinsically fast. The signal speed and dead time are limited by photon-detector. Though, for an integrating calorimeter considered for E158 this is not an issue.

The Cherenkov effect has a threshold which, for quartz, is $\beta=0.7$. Also, the emission of Cherenkov light is a directional process. For a particle with $\beta \approx 1$ traveling in quartz, the Cherenkov light is emitted in a cone at an angle of about $46^{\circ}$ to the direction of the particle. Thus the particles must pass the fibers within a narrow angular interval (whose maximum is about $46^{\circ}$ ) in order to produce a significant signal.

As a result, the noise from the induced radioactivity of the calorimeter material is significantly suppressed. No signal is generated for low energy uncharged particles such as gamma and neutrons. The velocity threshold restricts the nuclear decay products to be electrons in order to have any chance of being relativistic. Nuclear decays which emit $\alpha$ 's are invisible. Compton electrons from $\gamma$-e scattering can generate signal but must have a minimum $0.7 \mathrm{MeV}$ to generate light. Thus the device is blind to low energy neutrons and nearly blind to radioactivation, which 
Figure 3.1: Fibers used for the Moller detector.

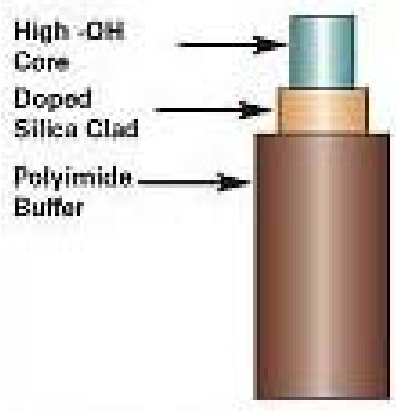

is an important advantage for high intensity applications like ours.

One of the most severe requirements of the experiment is the large radiation resistance. For the expected scattered electron rate in the detector, a dose of 10 Mrad per week is expected [4]. Thus the quartz as an active medium is the most favorable choice because of its high radiation resistance. High purity quartz has been reported to withstand radiation levels up to $3 \mathrm{Grad}$, with the transparency loss of less than $2 \%$ in the wavelenght range $300-425 \mathrm{~nm}$ [49].

The particular fibers used in the Moller detector are Polymicro FVP800880930. The fiber material is amorphous silica. The core is $0.8 \mathrm{~mm}$ thick, and is $\mathrm{OH}$ enriched which gives optimal transmission for bialkali phototubes. The reflective coating is also doped silica for radiation hardness. Finally, there is a polymide coating. These materials in general and the fibers made of these materials are known to be radiation hard.

Copper is chosen as the absorber material for the detector which provides an optimal absorber for several reasons: it is strong, easy to machine and relatively inexpensive. The $\mathrm{Cu}$ radiator presents no radiation damage problems. In addition, $\mathrm{Cu}$ has good thermal conductivity to make it easy to dissipate the $50 \mathrm{~W}$ power deposited in the detector. Also, the choice of this relatively low-Z material reduces the neutron production. 


\subsubsection{Detector Geometry}

The Moller detector is a $25 \mathrm{~cm}$ long $\mathrm{Cu}$ cylinder with an inner radius of $13.7 \mathrm{~cm}$ and an outer radius of $24.8 \mathrm{~cm}$. Almost $10 \%$ of the 'active' volume is occupied by the quartz fibers. The inner and outer radii of the active region are $15 \mathrm{~cm}$ and $23.5 \mathrm{~cm}$ respectively as shown in Fig. 3.2.
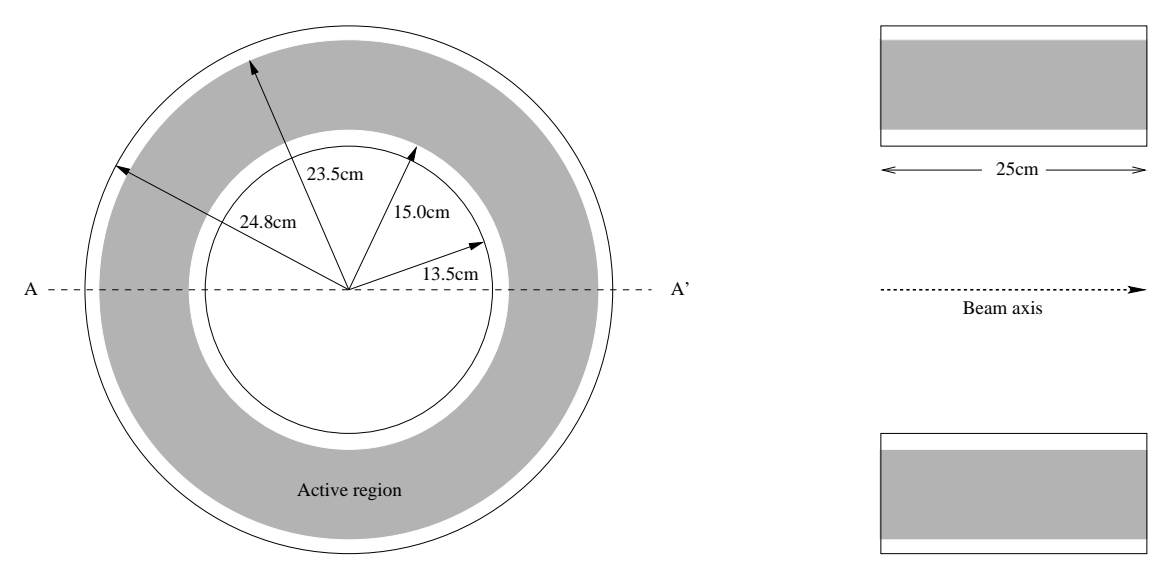

Section AA'

Figure 3.2: Moller detector outline

The radiation length of the detector changes with the radius and is $15.8 \mathrm{RL}$ (neglecting the RL of quartz) at the maximum radius. This is a compromise between having enough lengths to keep the fluctuations due to the shower development to at worst $\sim 10 \%$ level while minimizing the thickness where a pion can interact. The beam is concentric with the detector. All of the Moller electrons are focused onto the $\mathrm{Cu}$. They are approximately parallel to the beam.

The detector cylinder is composed of $100 \mathrm{Cu}$ wedges oriented at $45^{\circ}$ relative to the beam axis as shown in Fig. 3.3. (Note that the Fig. 3.3 also shows $\mathrm{Cu}$ wedges of $e P$ detector which will be discussed later.) At the outer diameter the wedges are 
$1.1 \mathrm{~cm}$ thick. The plates are mounted on an inner $A l$ cylinder of $13.7 \mathrm{~cm}$ radius. Between each wedge is a layer of $0.93 \mathrm{~mm}$ diameter quartz fibers. This geometry allows for reasonably uniform sampling and orients the fibers at $45^{\circ}$ relative to the beam to optimize the light output. At this angle, some of the Cherenkov radiation will be emitted in a direction parallel to the fibers.

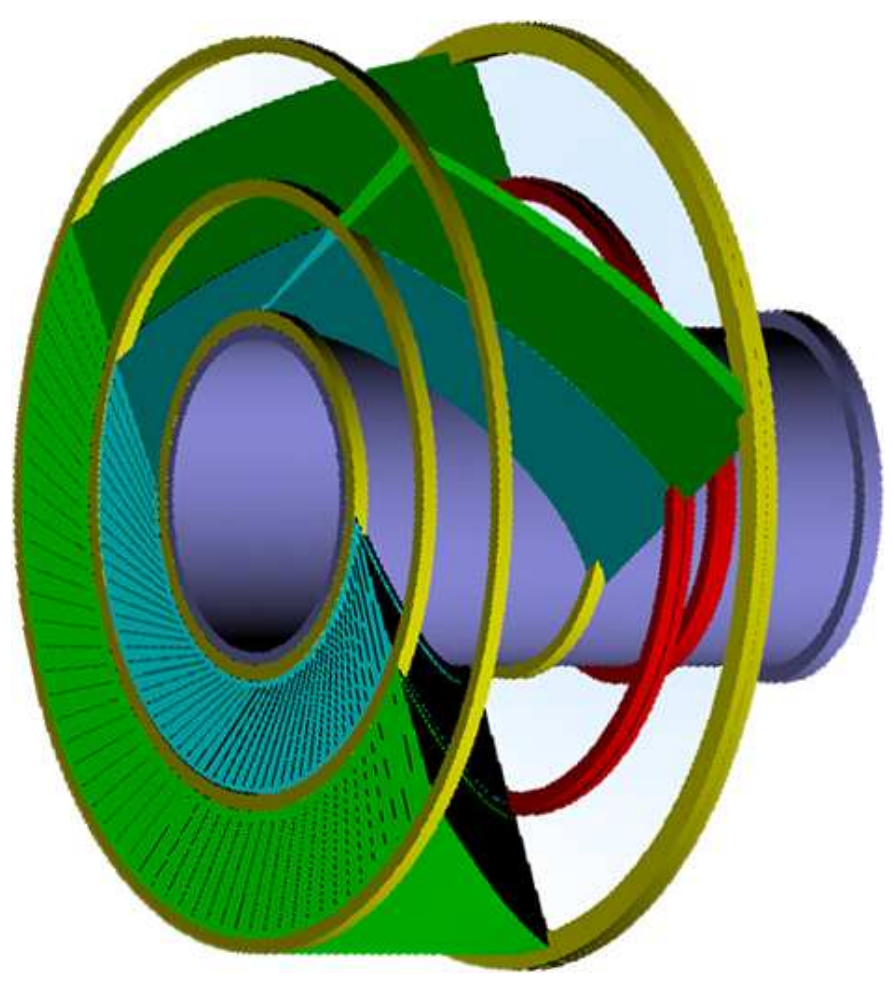

Figure 3.3: Moller and $e P$ detector cad diagram

A detailed study of the geometry of the $\mathrm{Cu}$ wedges is shown in Fig.3.4. The intersection of a cylinder and a plane oriented at $45^{\circ}$ relative to the axis of the cylinder is

$$
x^{2} / 2+y^{2}=r^{2}
$$




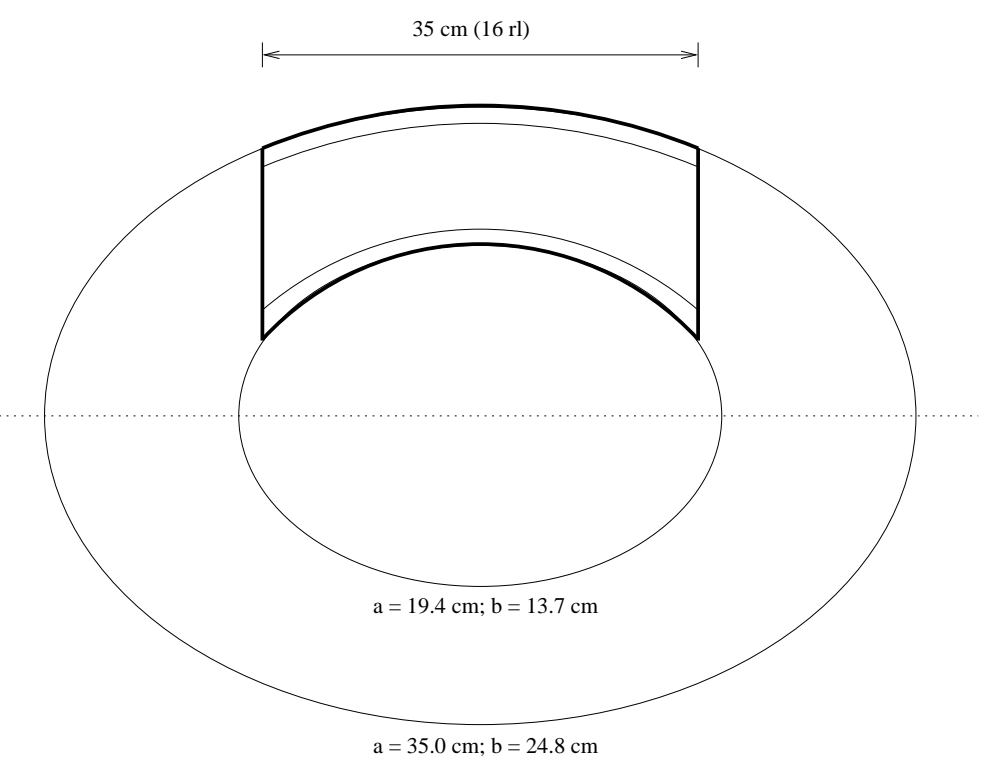

Figure 3.4: The shape of the $\mathrm{Cu}$ plate for the Moller detector.

where $r$ is the radius of the cylinder, $y$ is the radial direction, and $x$ is the orthogonal direction in the plane of the wedge. The total range of $x$ must be $\sqrt{2} t$, where $t$ is the thickness of the detector. In our case, the wedges are $35 \mathrm{~cm}$ wide. A groove is etched on each plate to accommodate the fibers. Fig. 3.5 shows the $\mathrm{Cu}$ plate and a layer of fibers.

Each layer of fibers is $8.5 \mathrm{~cm}$ wide at the center and consists of 91 individual lengths. The layers are divided into three sections $(2 \mathrm{~cm}, 3 \mathrm{~cm}$, and $3.5 \mathrm{~cm})$ by collecting the fibers in three groups (as shown in Fig. 3.5). Ten wedges (and hence 10 fiber layers) are connected together to form a single unit for mounting. The fibers from these 10 layers are collected in 5 bundles. The outer $2 \mathrm{~cm}$ of fibers from 5 layers are connected. Similarly, the middle $3 \mathrm{~cm}$ of fibers from the same 5 layers are bundled together. For the inner $3.5 \mathrm{~cm}, 10$ layers are collected. 


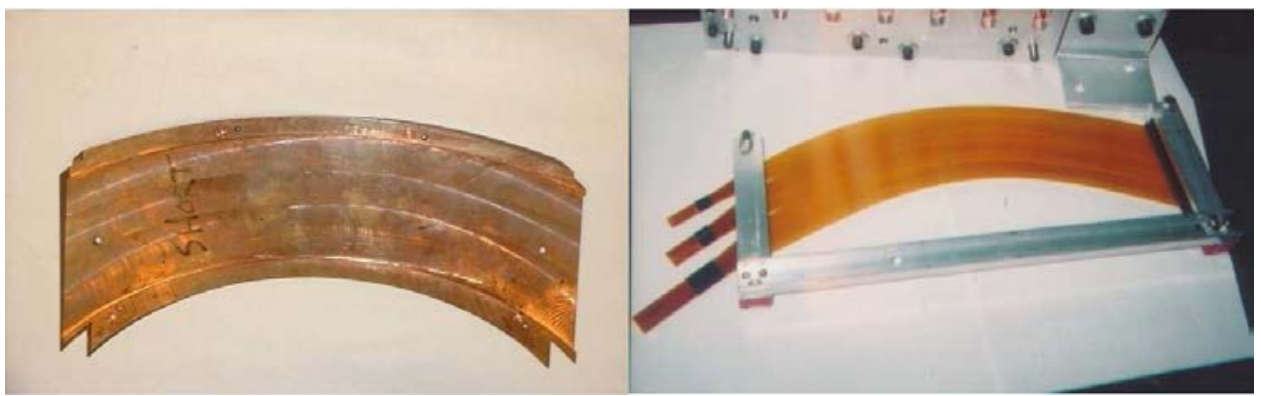

Figure 3.5: Moller $\mathrm{Cu}$ plate and a layer of fibers

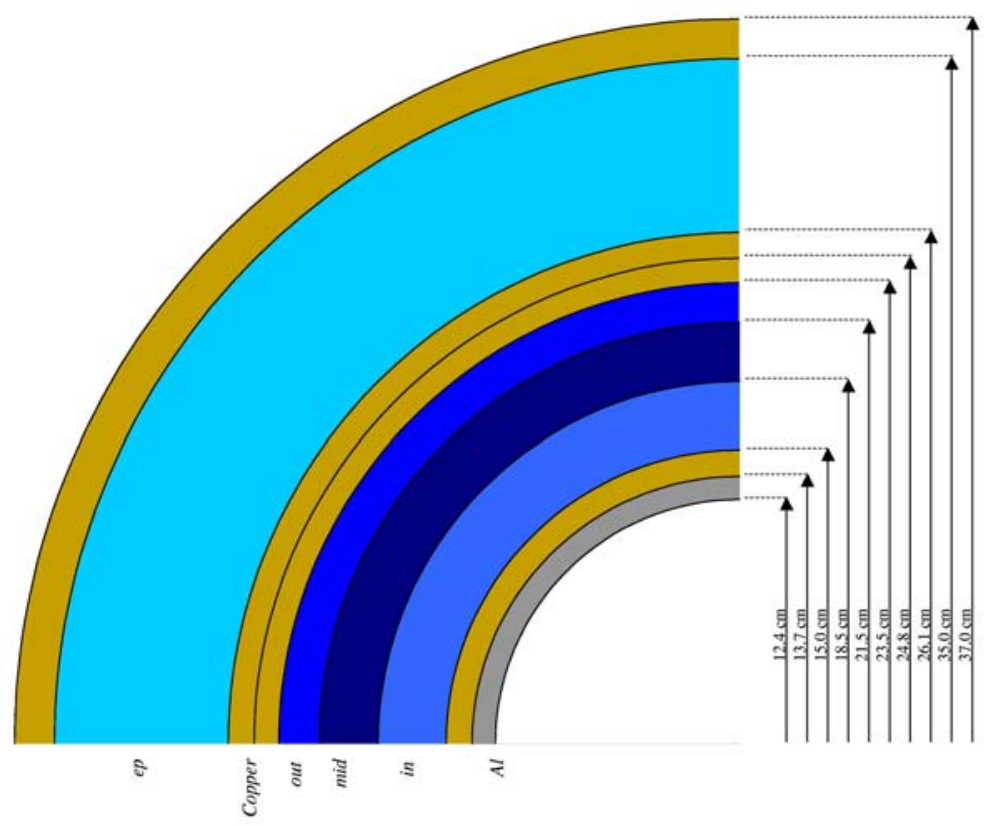

Figure 3.6: Radii of active regions of Moller and $e P$ detectors.

The arrangement of fibers, as explained above, provides both radial and $\phi$ segmentation. It divides the active volume in three rings (inner, middle, and outer) as shown in Fig. 3.6. The outer and the middle rings include 20 fiber 
bundles each, and the inner ring includes 10. The fibers are then connected to light guides reaching the photomultiplier tubes (PMT). The light guides and the mirrors are made from Alzak sheets $(0.5 \mathrm{~mm}$ thick). The PMTs are arranged in two circles of radii $67.3 \mathrm{~cm}$ and $75 \mathrm{~cm}$. The light guide assemblies are shown in Fig. 3.7.
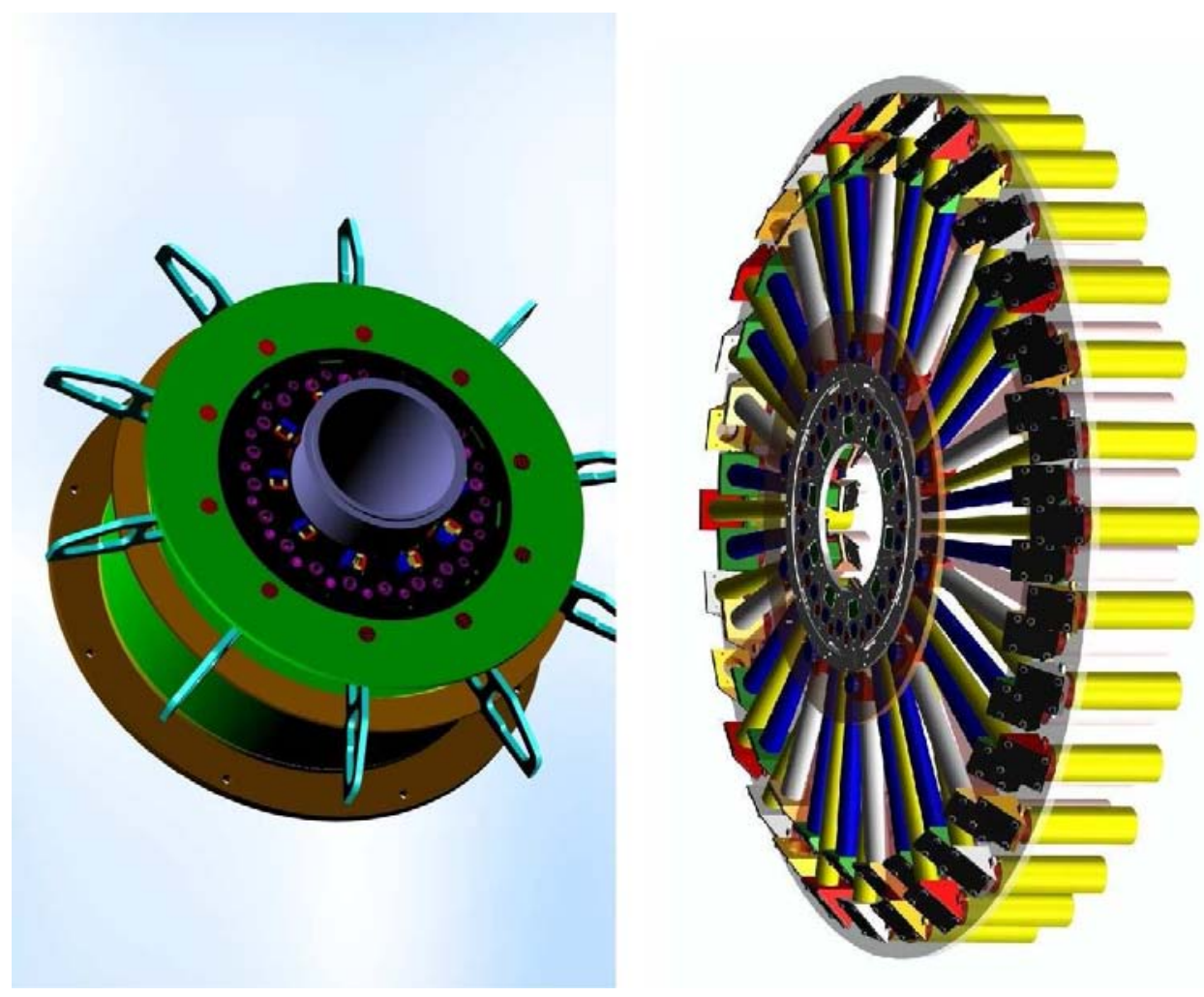

Figure 3.7: (left) Back plate of the detector assembly showing the cookies where the fibers are collected. (right) Mirrors, light guides and cylinders that hold the PMTs. 


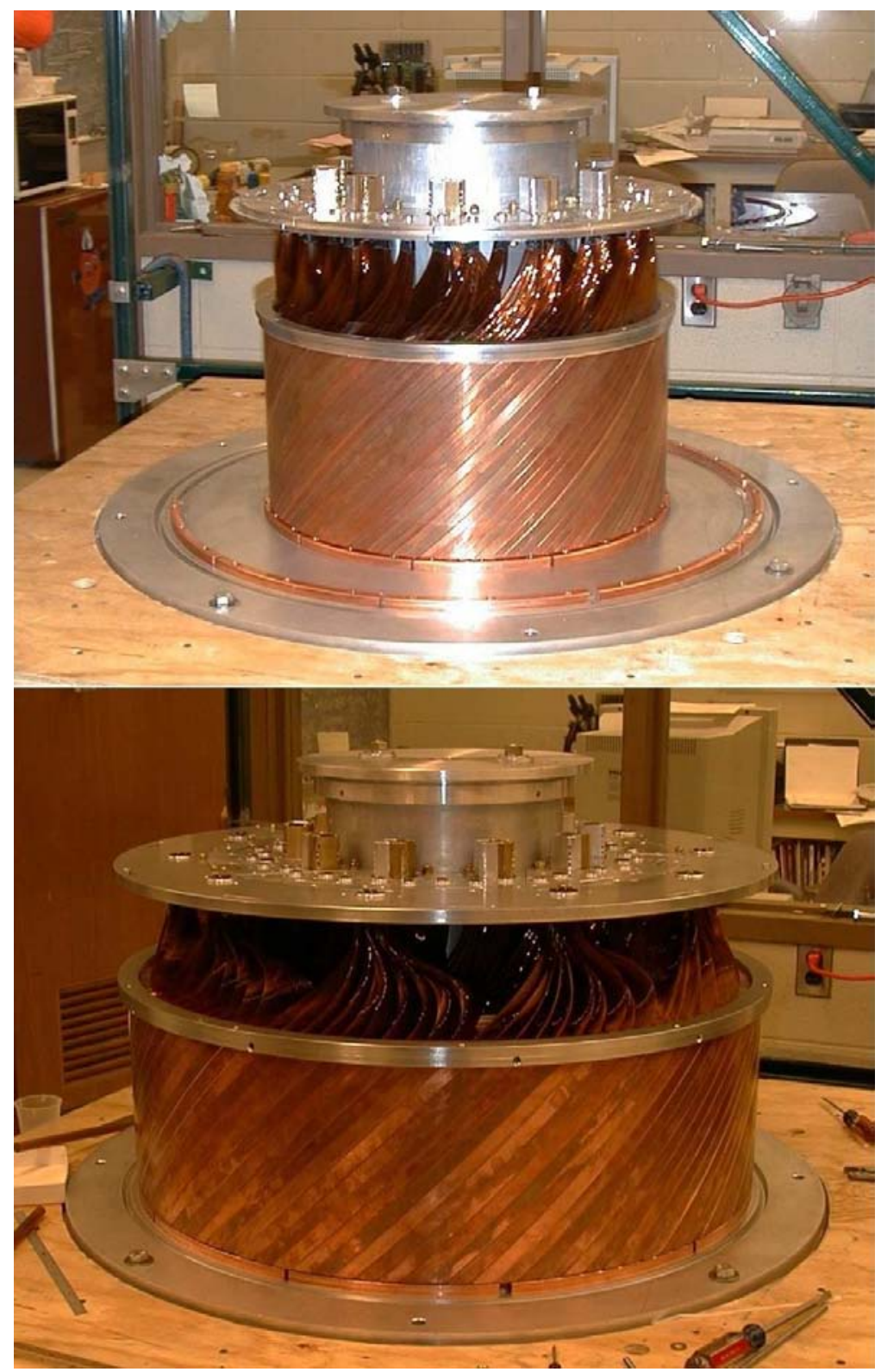

Figure 3.8: Complete Moller and eP detectors. 


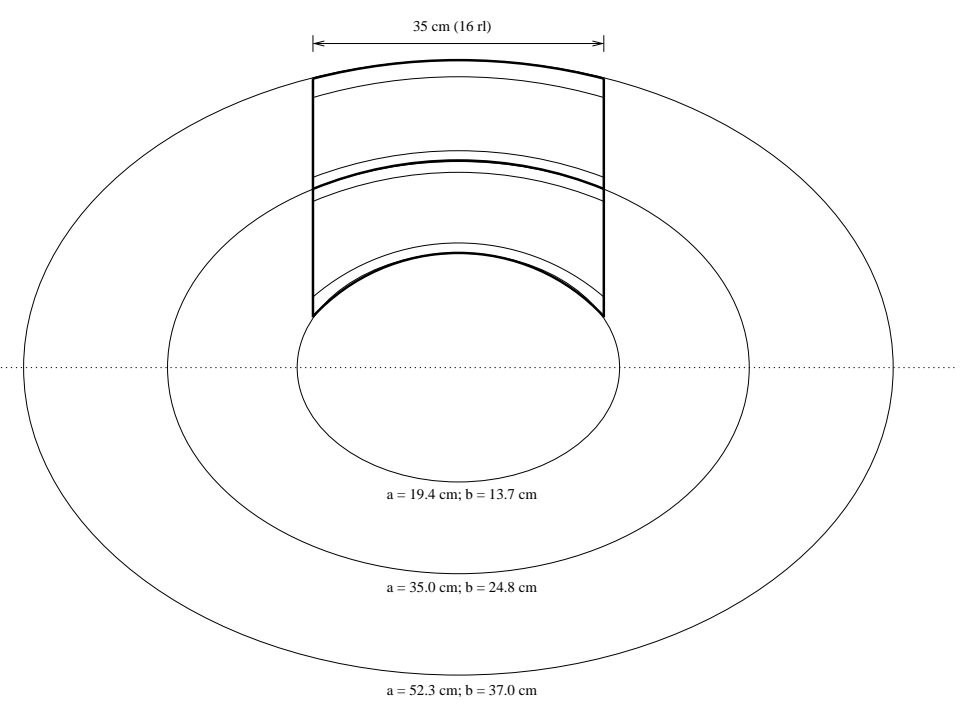

Figure 3.9: The shape of a $\mathrm{Cu}$ plate for the $e P$ detector.

\section{$3.2 \quad e P$ detector}

The $e P$ detector is also a quartz fiber calorimeter, and is similar in construction to the Moller detector. The inner and the outer radii of the $\mathrm{Cu}$ cylinder, in this case, are $24.8 \mathrm{~cm}$ and $37 \mathrm{~cm}$ respectively. Less than $2 \%$ of the acitve volume is occupied by the quartz fibers. The inner and the outer radii of the acitve region are $26.1 \mathrm{~cm}$ and $35 \mathrm{~cm}$ respectively, as shown in Fig. 3.6.

The detector is composed of $100 \mathrm{Cu}$ wedges, arranged around the Moller detector to form a cylinder. A detailed study of $e P \mathrm{Cu}$ wedge is shown in Fig. 3.9. The thickness of the plate at the outer diameter is $1.6 \mathrm{~cm}$, and the cutout to hold the fibers is $8.9 \mathrm{~cm}$ wide at the center.

The fibers used for the $e P$ detector are Polymicro FVP300330370, which have the same material and construction as the ones used for Moller detector, but have different thickness. The diameter of the core, in this case, is $0.3 \mathrm{~mm}$. To form one 


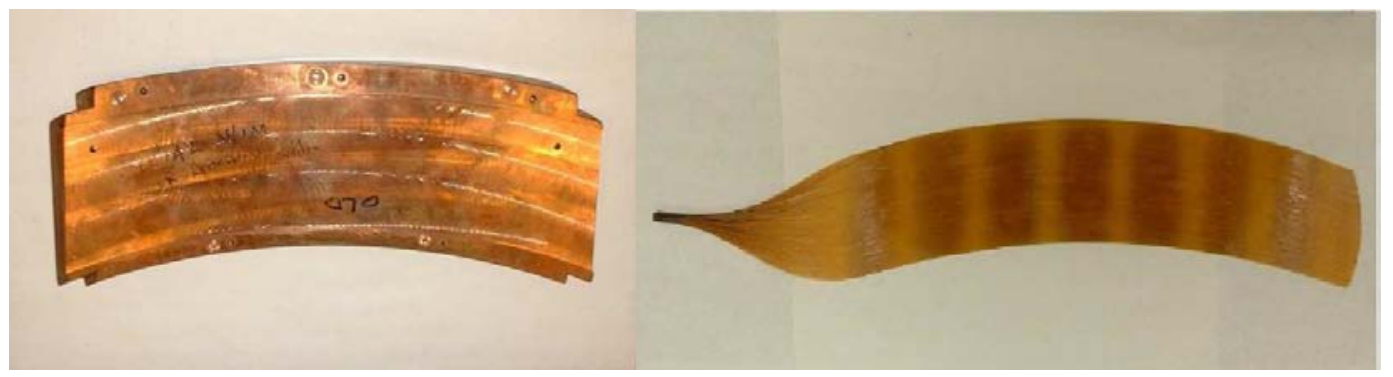

Figure 3.10: eP $\mathrm{Cu}$ plate and a layer of fibers.

layer, 237 individual lengths of the fibers are glued together as shown in Fig. 3.10.

Again, 10 wedges are combined to form one unit for mounting. All the fibers from the 10 layers are collected at one place, thus, there is no radial segmentation in the $e P$ detector. The whole $e P$ detector has 10 bundles of fibers. Fig. 3.7 shows the positions of the $e P$ fibers and light guides.

\subsection{Putting It All Together}

The complex design of the detector as described in the previous sections makes it very challenging to mount fibers. Amorphous silica fibers are extremely delicate and require great care to work with. A minor cut in the polymide cladding can cause the fiber to break while bending, and our geometry required the fibers to be bent at large angles after they leave the $\mathrm{Cu}$ plates as can be seen in the picture shown in Fig. 3.8. This section provides the techniques used in processing fibers from cleaving to bundling. 


\subsubsection{Cleaving}

The process of cleaving the fibers is very crucial because the quality of the cleavage plane can affect the light yield significantly. The fibers used for the Moller detector ("Moller fibers") are thicker and more rigid compared to the ones used for the $e P$ detector (" $e P$ fibers"). Thus, different methods for cleaving are used for the two types.
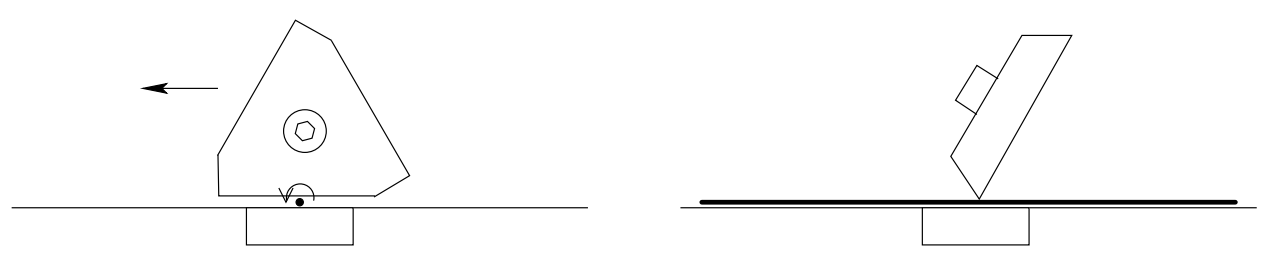

Figure 3.11: Schematic of the cutter used to cut the Moller fibers.

We developed a simple yet efficient method to cut a large number of Moller fibers while maintaining the quality of the cleavage plane. Fig. 3.11 shows the schematic of the "cutter", which employed a tungsten carbide blade normally used for metal cutting. To cut the fiber, it is placed under the blade and the blade is moved back and forth with increasing pressure while spinning the fiber at the same time. This way the fiber will split, (after 3-4 "strokes" on the average,) when a

Figure 3.12: Cleaved ends of Moller fibers.

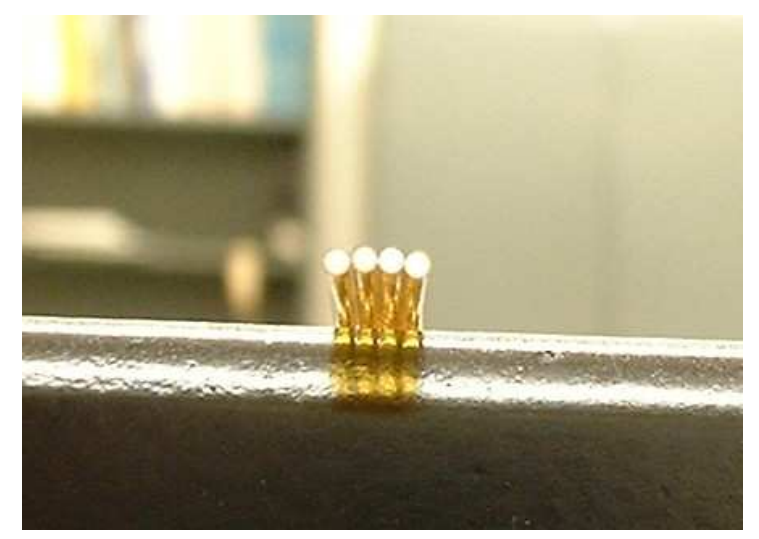


nice circular cut is obtained on the cladding. Note that the back and forth motion of the blade should not cause multiple cuts on the cladding and should not drag the fiber with it. (This requires some practice!) Fig. 3.12 shows a picture of the cleaved fibers.

The light yield from different cleavage planes was compared in a simple bench test setup. A radioactive source was used to generate Cherenkov light in the fibers, and the light was collected using a PMT. Properly cleaved fibers using the tungsten carbide blade were found to have optimal light output.

The $e P$ fibers, on the other hand, are thinner and more delicate to cut as compared to the Moller fibers. Thus the tungsten carbide cutter could not be used in this case. A cutter installed with a sharp diamond wedge/blade was used instead.

To cut the $e P$ fibers, a small cut is made at the desired length of the fiber without slicing or damaging its core. Then, the fiber is pulled at both ends forcing the fiber to break where the cut was made. Again, the method requires some practice! Fig. 3.13 shows a picture of fiber ends collected from one layer of $e P$ fibers.

Figure 3.13: Cleaved ends of $e P$ fibers.

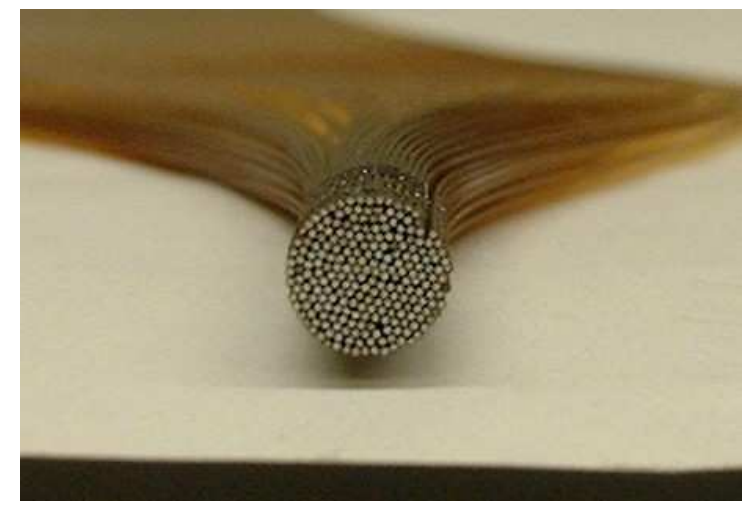




\subsubsection{Constructing Layers}

The next challenging step was to put the fibers in the elliptical channels cut in the $\mathrm{Cu}$ plates. Again, Moller and $e P$ fibers were treated differently because of their different physical characteristics.

As mentioned earlier, the Moller detector active volume was subdivided into three distinct rings (Fig. 3.6). To obtain this segmentation, and to cover the whole space properly, 91 fibers of 6 different lengths were arranged in three groups of 22, 32 and 37, for each layer. Fibers in each subset were glued at one end in two different layers as shown in Fig. 3.14. A fine copper mesh was placed between the layers to give an extra strength to the glued end. A taflon mold was constructed for this purpose. These double layer assemblages of fibers were put into one layer using a specially designed jig which is shown in Fig. 3.15.
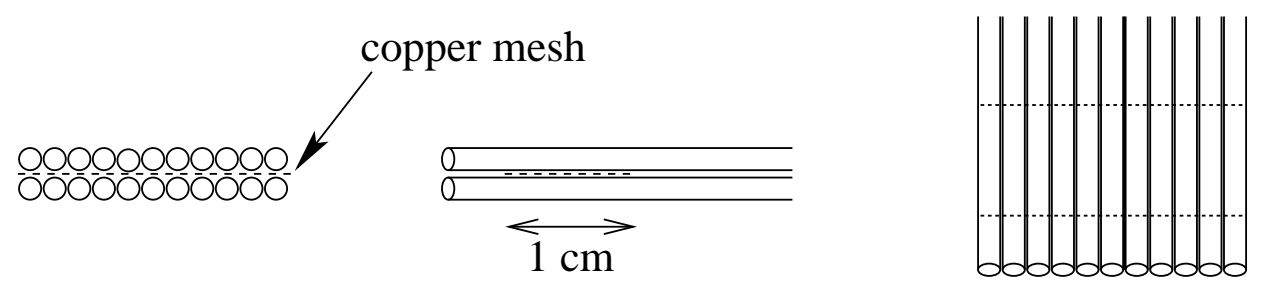

Figure 3.14: Glued ends of Moller fibers.

The complete process of creating one layer from three glued sets is shown in Fig. 3.16. First, fibers are inserted in the jig and pressed from the top using a 1 mm thick $A l$ plate (Fig 3.16(a)) which forces the fibers in an elliptical band similar in shape to the channel etched in the $\mathrm{Cu}$ plate. The fibers are then clamped from both sides as shown in Fig. 3.16(b), and taken out of the jig. The clamp retains the shape of the layer. Next, the fiber layer is placed in the channel in the $\mathrm{Cu}$ plate and a $0.5 \mathrm{~mm}$ thin $\mathrm{Cu}$ 'shim', which has exactly the same shape as that 


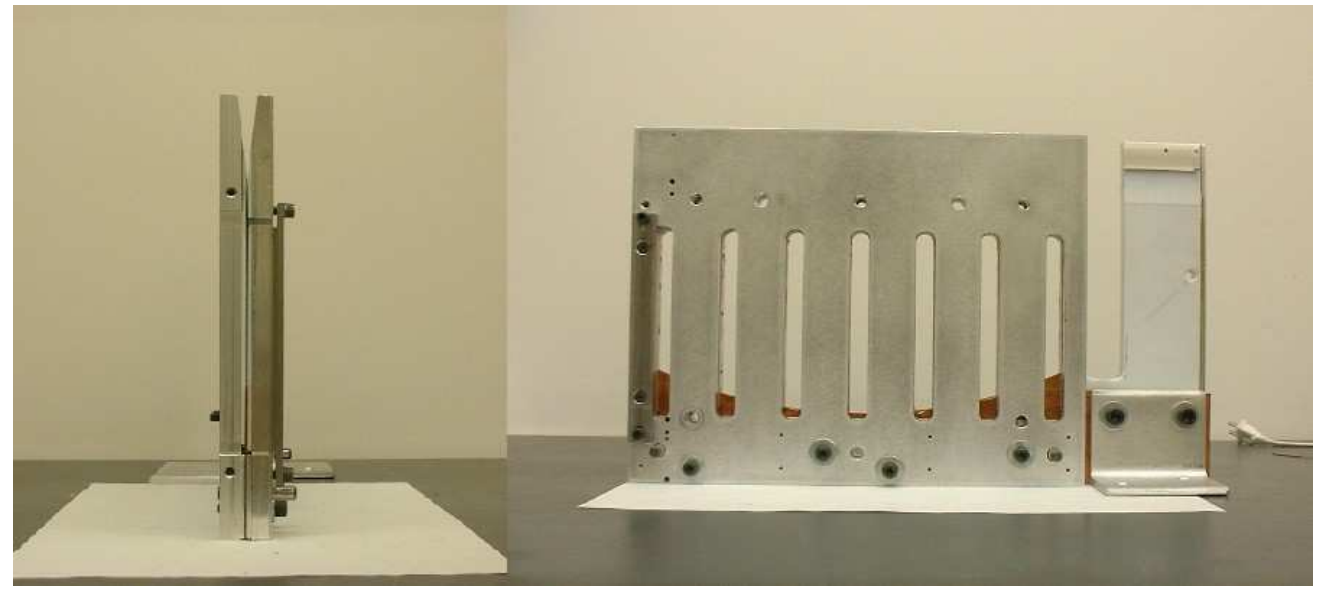

Figure 3.15: The jig used to produce layers of fibers for Moller detector.

of the etched channel, is put on top of the fibers. Finally, the next $\mathrm{Cu}$ plate is placed on the top and screwed permanently. The small unevenness in the $\mathrm{Cu}$ shim produces a 'spring' effect and keeps the fibers firmly in one plane. The clamp is then removed and the fibers are pulled from the longer ends to align the free ends with the edge of the plate.

The channel cut on the $\mathrm{Cu}$ plate is bounded by two ellipses defined by the inner and outer radii of the active volume (see Fig. 3.4). This is necessary in order to have a cylindrical active volume. But the fibers arranged as described do not cover the whole channel and empty spaces are left at the inner corners of the plate as sketched in Fig. 3.17. Thus, effectively, the active volume is not a perfect solid cylinder.

For $e P$ detector, 237 individual lengths were used to cover one plane. All these fibers were, first, glued at one end to create one circular bundle as shown in Fig. 3.13. The jig used to create a layer from these glued fibers was similar to the one used for Moller fibers but with smaller spacing to accommodate thinner fibers. 


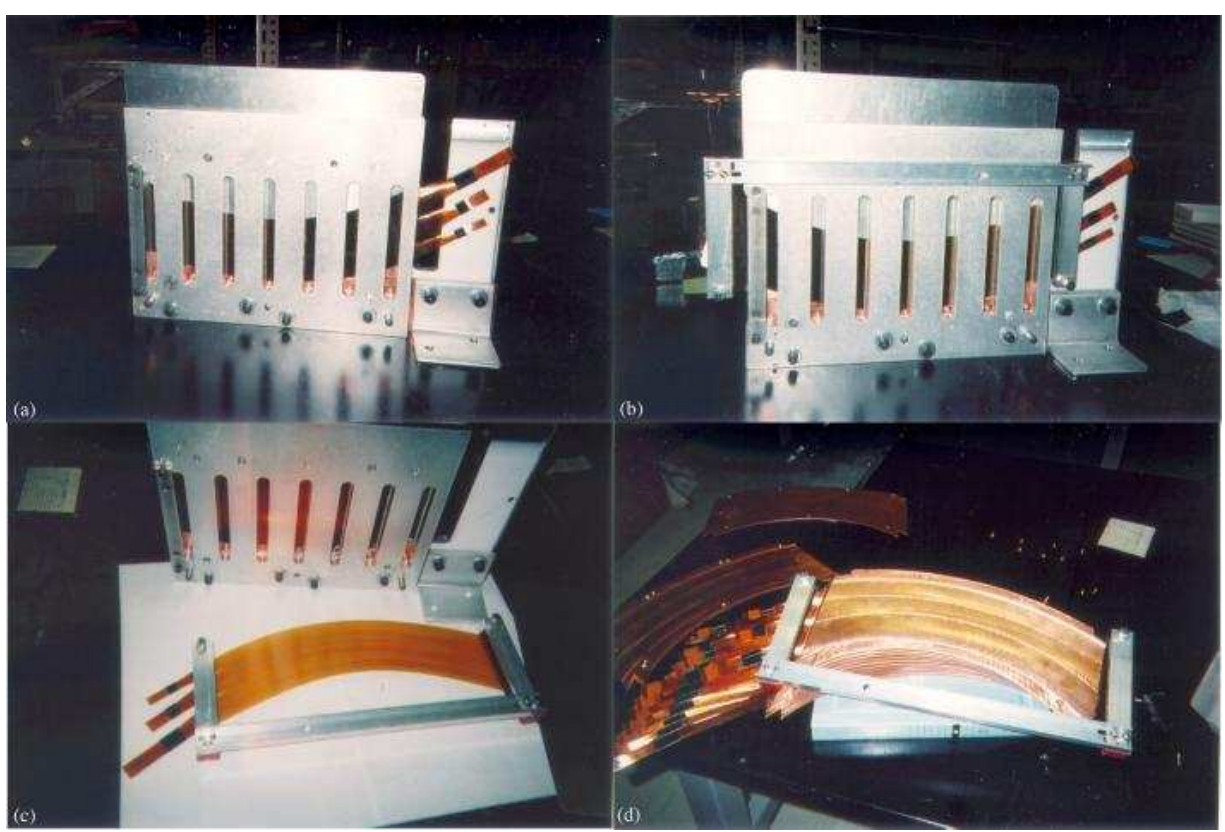

Figure 3.16: The complete process of creating layers of Moller fibers. (a) Fibers are inserted and pressed from the top, (b) fibers are clamped, (c) clamped fibers taken out from the jig, (d) fiber are inserted in $\mathrm{Cu}$ plate.

Figure 3.17: Empty spaces in the channel in a Moller $\mathrm{Cu}$ plate.

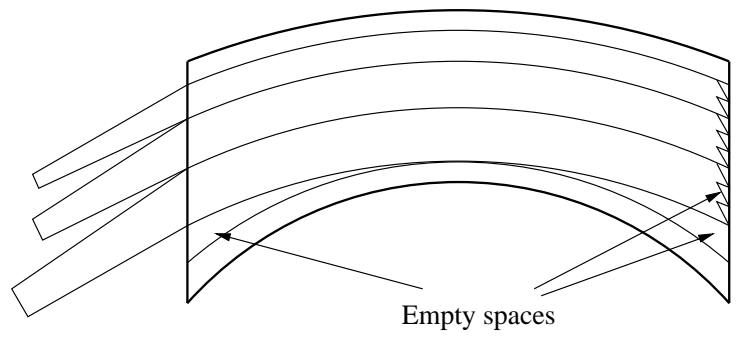

Since the $e P$ fibers were much more flexible and greater in number, the clamping method could not be used. They were glued instead while in the jig. Fig. 3.18 shows the jig with the fibers in, and Fig. 3.10 shows one finished layer. Once the layers are prepared, it is straight forward to put them in $\mathrm{Cu}$ plates. No $\mathrm{Cu}$ shims are necessary in this case.

For each detector the basic mounting unit consisted of ten quartz fibers layers 
Figure 3.18: The jig used to

produce layers of fibers for

$e P$ detector.

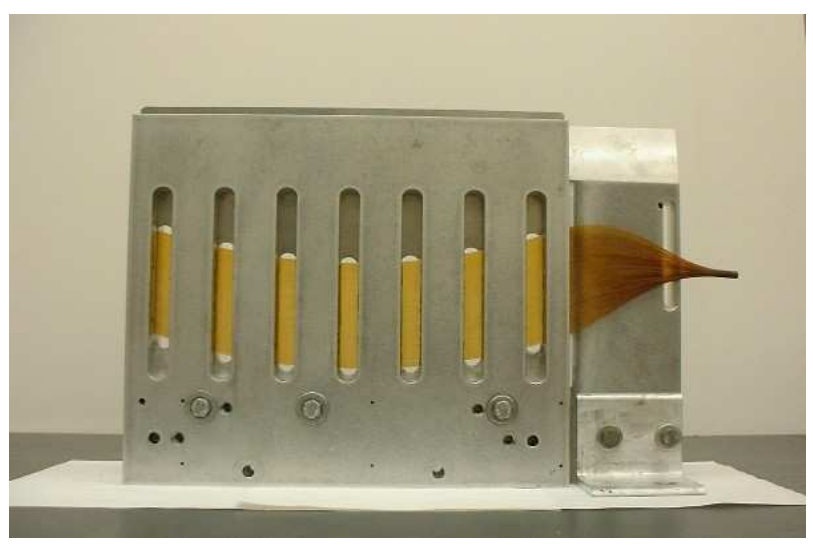

interspersed in ten $\mathrm{Cu}$ plates. (There was a $0.5 \mathrm{~mm}$ thin $\mathrm{Cu}$ layer on top of each basic unit to hold the 10th fiber layer.) These units of ten were arranged around an $A l$ pipe to complete the cylinder. As an important precaution, all the edges of each $\mathrm{Cu}$ plate were burnished before inserting the fibers to avoid damaging the cladding from the sharp corners.

\subsubsection{Bundling the Fibers}

Perhaps the most difficult and delicate step in assembling the detector was to bundle the Moller fibers from different layers. Special clamps ("cookies") were designed for this purpose.

As mentioned in the previous section, each fiber layer for the Moller detector was divided into three sections, which practically divided the active volume in three rings. The inner most section (relative to the center of the cylinder) in each layer had 37 fibers. To construct one bundle of fibers for the inner ring 10 such sections from consecutive layers were combined together. Thus the total number of fibers in each inner bundle were 370 arranged in a rectangular array. This made it extremely hard to bend the whole collection together because of the stiffness of 
the fibers. The clamps designed to hold such bundles had three separate sections and were mounted directly on the central Al pipe. These clamps are pictured in Fig. 3.20.

For the middle and the outer rings the bundles were constructed by combining fibers from 5 consecutive layers. Each bundle in the middle ring contained 160 fibers arranged in a rectangular array of $16 \times 10$. Fig. 3.19 shows the cookies used to hold these fibers. The cookies used for the fibers in the outer ring are similar in construction but smaller in size to hole an array of $11 \times 10$ fibers. All these cookies were held in position using cookie plates mounted directly on the central pipe. Each cookie plate held four cookies, and ten such plates constituted a complete ring. Fig. 3.20 show the pictures of the cookies and the plates.

The cookies for the $e P$ fibers were designed to hold ten circular bundles of fibers collected from ten consecutive layers as shown in Fig. 3.20. The whole $e P$ ring contained ten such cookies. Fig. 3.7 shows the cad diagram of the back of the detector showing the positions of all the Moller and the $e P$ cookies.
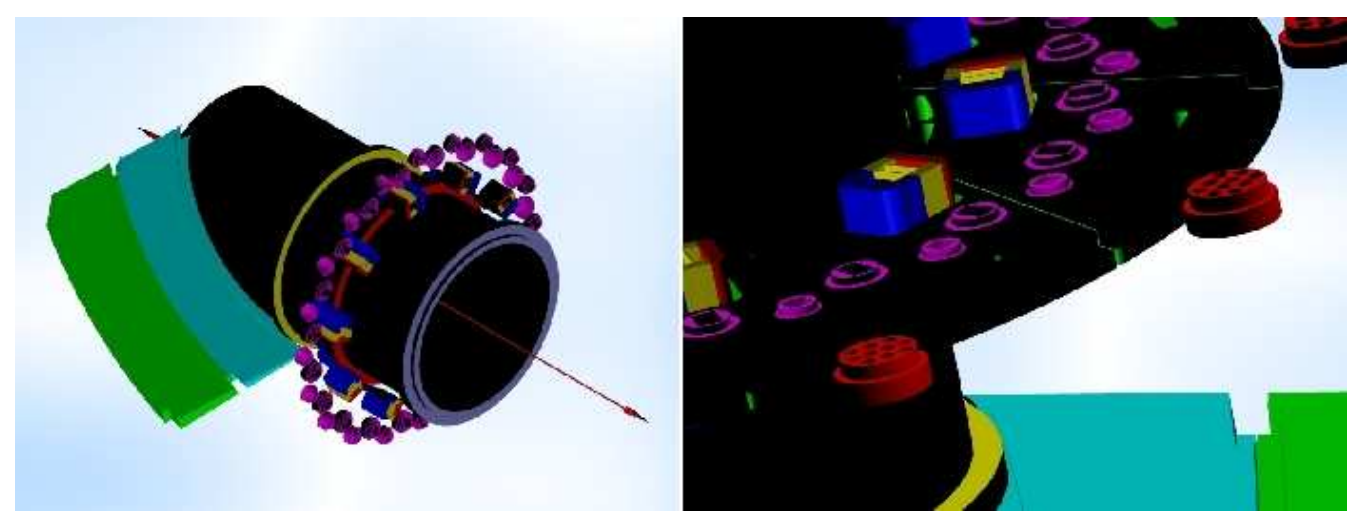

Figure 3.19: cookies and cookie plate. 


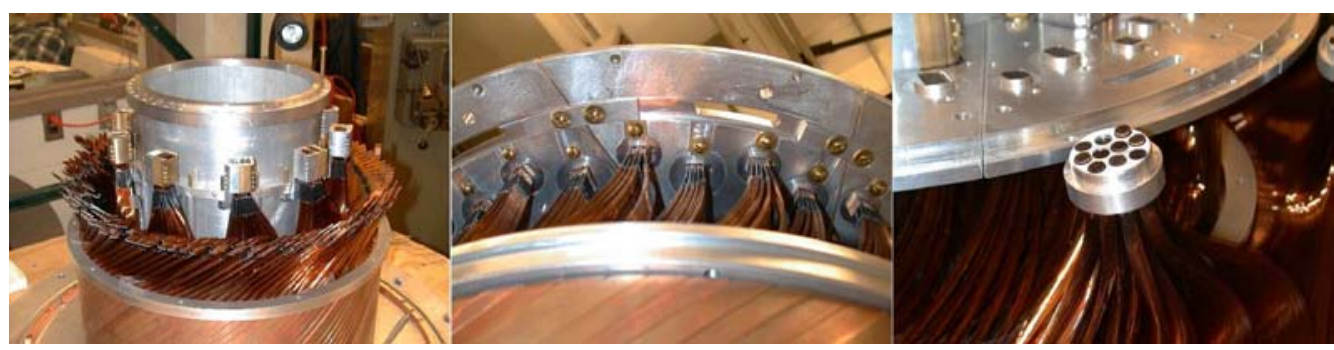

Figure 3.20: (top) Inner, middle and outer, and eP cookies; (right) Elegant view of the back of the detector showing the cookies and cookie plates!

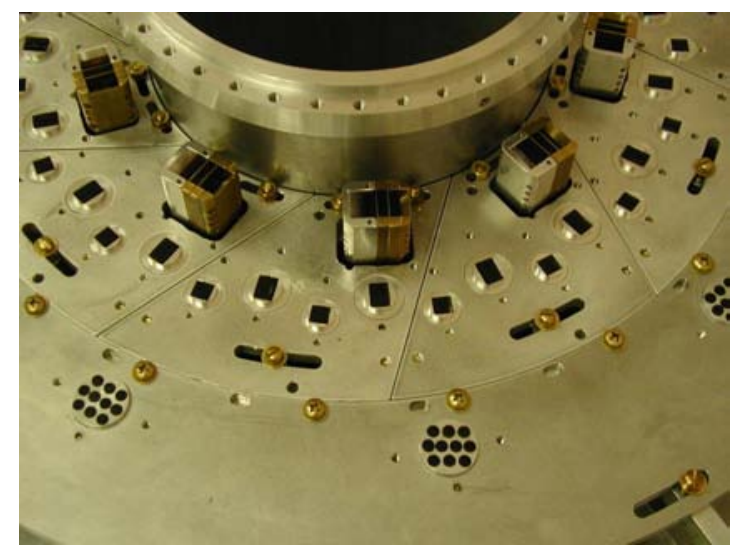

\subsubsection{Mirrors, Light Guides, and PMT Assemblies}

In order to avoid the high flux of particles hitting the dynodes, the phototubes were placed far from the beam pipe, behind the lead shielding. The light collected from the fibers from the back of the detector was transfered to the PMTs through periscope like combination of mirrors and air light guides.

Highly polished Al sheets (ALZAK, $0.5 \mathrm{~mm}$ thick) were used to build the mirrors and light guides. The loss of light through reflection from the polished surface was found to be less than $5 \%$ in a bench-test measurement. Since the light from the fibers is well collimated the light requires few bounces and this much reflectivity is ample. Fig. 3.21 shows a schematic diagram of the periscope. The conical shape of the light guide further minimizes the number of reflections inside the pipe. 


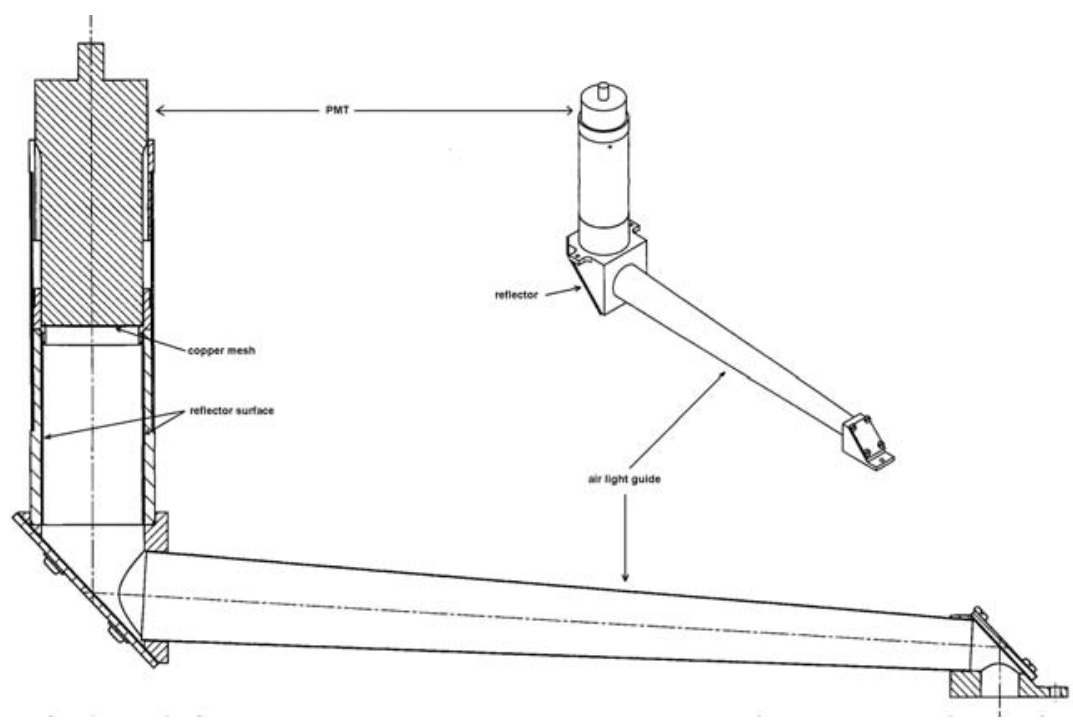

Figure 3.21: Mirrors, light guide and PMT assembly.

PMTs were buried $9 \mathrm{~cm}$ further into the lead shielding. The assemblies to hold the PMTs were designed to become part of the periscope, but could be inserted (and removed) from the back of the lead shielding at any stage. Fig. 3.21 shows a schematic of such assembly.

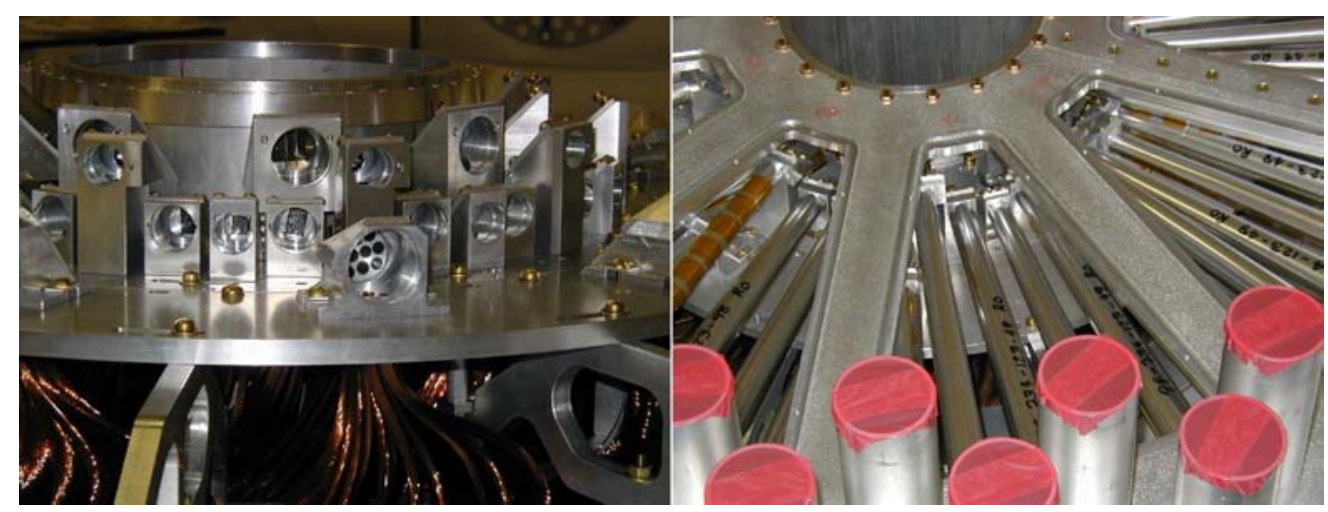

Figure 3.22: (left) Mirrors; (right) air light guides; The phototube assemblies are inserted inside the cylinders shown here. 


\subsubsection{Lead Shielding}

The phototubes can withstand on the order of 0.1 Mrad of radiation dose. A preliminary GEANT simulation showed that the field behind the Moller detector was 0.10 times the field at shower maximum [51]. At the location of the phototubes the field is reduces another factor of 1000. The lead shielding shown in Fig. 3.23
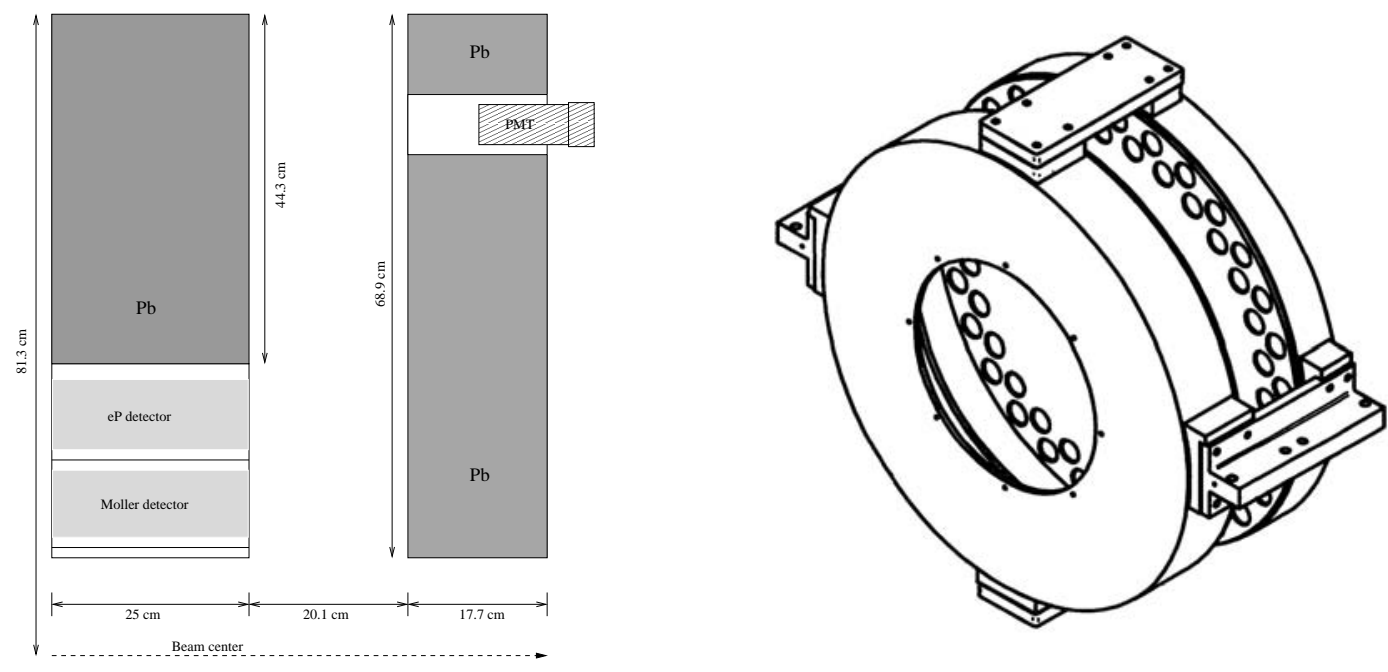

Figure 3.23: (left) Schematic diagram of the lead shielding; (right) sketch of the front and back lead "donuts".

Figure 3.24: Complete E158 detector with the lead shielding (before the light tight jacket was installed).

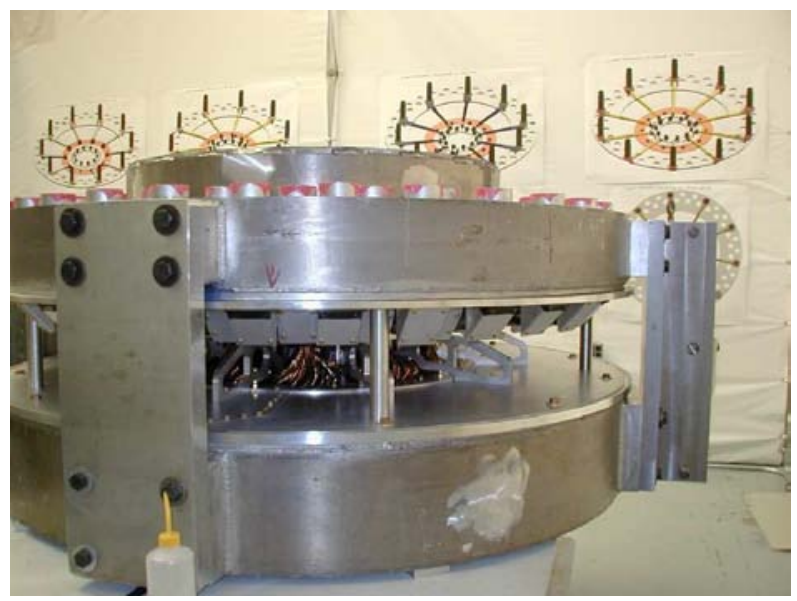


reduces the radiation by another factor of 25 [52]. By burying the PMTs further down into the lead, a reduction by an amazing factor of 150 is obtained. This leads to a total absorbed radiation dose throughout the experiment of as low as $1 \mathrm{rad}[52]$. Thus the level of radiation at the PMTs present no problem, either with producing unwanted signal in the tubes or degrading the performance of the tubes.

\subsection{Linearity of the Photomultiplier Tubes}

The photomultiplier tubes ${ }^{1}$ used in the detector are 10 stage tubes with $51 \mathrm{~mm}$ diameter borosilicate glass windows and $46 \mathrm{~mm}$ bialkali photocathodes. These PMTs have fast time response and a typical gain of $1.0 \times 10^{6}$. The bases selected for the tubes have the voltage distribution ratios given in the Fig. 3.25. At the highest beam intensity, any individual PMT from the area of highest signal rate receives up to twenty million photons per spill. This rate is high for the PMTs, so to prevent cathode saturation (which would result in a sizable nonlinearity), a copper wire mesh is placed on each PMT's cathode to allow charge to quickly redistribute over the cathode. This mesh also significantly reduces the amount of light entering the PMT.

\begin{tabular}{|c|c|c|c|c|c|c|c|c|c|c|c|c|}
\hline Electrodes & $\mathrm{K}$ & Dy1 & Dy2 & Dy3 & Dy4 & Dy 5 & Dy6 & Dy7 & Dy8 & Dy9 & Dy10 & $P$ \\
\hline Ratio & 2 & 1 & 1 & 1 & 1 & \begin{tabular}{l|l} 
& 1 \\
\end{tabular} & \begin{tabular}{l|l}
1.2 \\
\end{tabular} & \begin{tabular}{|l|l|} 
& 1.5 \\
\end{tabular} & \begin{tabular}{|l|l|} 
& 2.2 \\
\end{tabular} & \begin{tabular}{l|l} 
& 3.6 \\
\end{tabular} & \begin{tabular}{l|l} 
& 3 \\
\end{tabular} & \\
\hline Capacitance $(\mu F)$ & 0.001 & 0.001 & 0.01 & 0.01 & 0.01 & 0.01 & 0.01 & 0.01 & 0.01 & 0.01 & 0.02 & \\
\hline
\end{tabular}

Figure 3.25: Dynode voltage distribution ratios.

\footnotetext{
${ }^{1}$ Model R2154 manufactured by Hamamatsu Photonics.
} 
Figure 3.26: Schematic

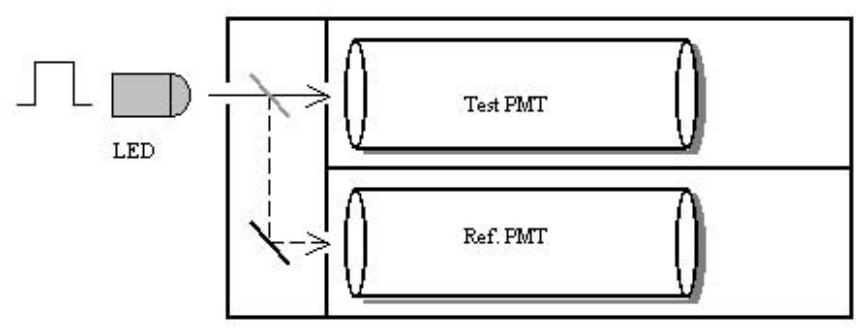

The linearity of each PMT was tested in a bench-test setup before installation. Fig. 3.26 shows the schematic of the apparatus used to test the PMTs. It employed a bright blue LED to generate light pulses of $300 \mathrm{~ns}$ duration. The light was split into two channels, one for the PMT being tested and the other for a reference PMT used to measure the light level. The ref. PMT received ten times less light than the test PMT. The output signals from both PMTs were fed to standard CAMAC ADC channels which are known to be linear to a percent level over the whole range.

The output for each PMT was plotted against the light level, and the linearity was calculated from this curve, using two different definitions:

$$
\begin{aligned}
\operatorname{lin}_{1} & =\frac{L \times S^{\prime}(L)}{S(L)} \\
\operatorname{lin}_{2} & =\frac{S^{\prime}(L)}{S^{\prime}\left(L_{\circ}\right)}
\end{aligned}
$$

where $L$ is the input light level, and $S$ is the output signal from the PMT. Note that $2\left(\operatorname{lin}_{1}-1\right) \sim\left(\operatorname{lin}_{2}-1\right)$. The first definition is the actual correction to the measured asymmetry: $A_{\text {measured }}=\operatorname{lin}_{1} \times A_{\text {true }}[42,53]$. We require $\left|\operatorname{lin}_{1}-1\right|<0.005$. The second definition is the ratio of the slope of the $S$ vs. $L$ curve at any light level to the initial slope (or the slope at the origin). This definition was used mostly for the comparison of the test results. 
The Fig. 3.27 shows a typical set of plots produced for each PMT. The large plot in the upper left corner is the $S$ vs $L$ curve for different high voltages. Each PMT was tested at four different high voltages (HV). The PMTs were assumed to produce $0.5 \mathrm{~V}$ (into $50 \Omega$ ) output signal (300 ns wide) at the light levels expected $\left(L_{\text {exp }}\right)$ in the detector channels during the experiment. $L_{\text {exp }}$ was deduced using the data taken during the E158 commissioning run. The vertical dashed line in the graph represents $L_{\text {exp }}$. The horizontal dashed lines in the graph correspond to $0.3 \mathrm{~V}, 0.5 \mathrm{~V}$ and $0.75 \mathrm{~V}$ output signal heights. The first $\mathrm{HV}$ for any PMT was determined such that it produced $0.5 \mathrm{~V}$ at $L_{\text {exp }}$. The other two HVs were simply $\pm 30 \mathrm{~V}$ of the first HV. The fourth HV was selected such that the PMT produced $0.5 \mathrm{~V}$ signal at $L_{\text {exp }}$ but with a $53 \%$ transmission filter in front of the PMT window.

In the bottom left graph of Fig. 3.27, $\operatorname{lin}_{2}$ is plotted as a function of $L$, whereas the bottom center graph shows $\operatorname{lin}_{2}$ vs $S$. The two plots on the right-hand side of the figure are the most important ones. These curves show $\operatorname{lin}_{1}$ vs $L$ - the bottom plot is merely a zoomed version of the top plot.

The PMTs for different detector rings were selected on the basis of the values of $\operatorname{lin}_{1}$ at $L_{\text {exp }}$ for each tube, obtained from the bench-test. The most linear tubes were used for the middle channels. The least linear tubes were used for the $e P$ ring because the light expected in the $e P$ detector was much smaller as compared to the light in the Moller channels. Fig. 3.28 shows the distribution of the linearity of all the PMTs at $L_{\text {exp }}$.

These bench-test results cannot give the final linearity correction to the physics asymmetry. The bench-test studies were performed to improve the linearity of the PMTs, (during the E158 commissioning run, the PMTs behaved 20-30\% nonlinear,) and then test and record all PMTs in their final improved base configurations as discussed here. As mentioned earlier, these tests used CAMAC ADCs instead 

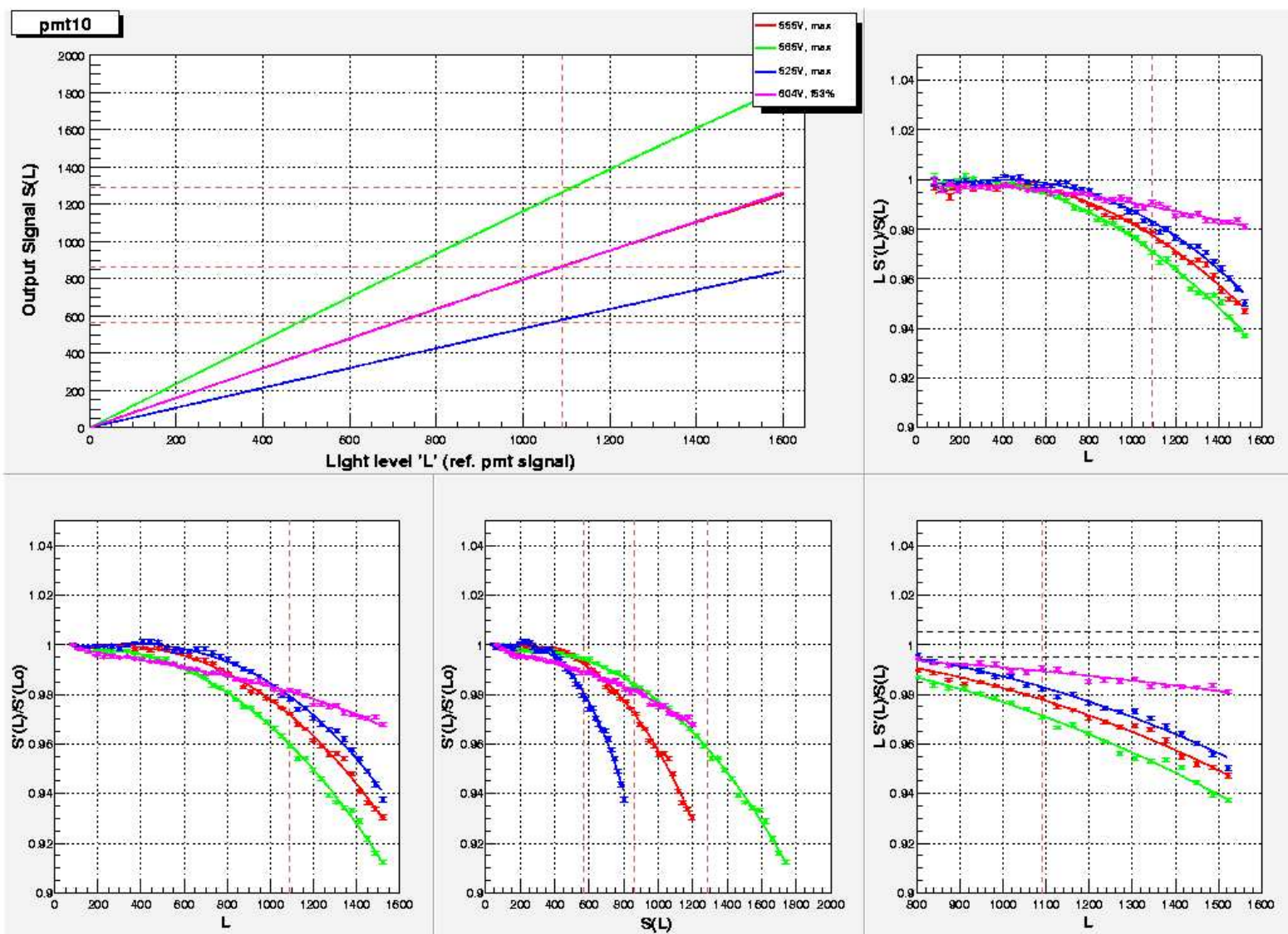

i.

Figure 3.27: Typical set of linearity plots produced for all PMTs in the benchtest. 

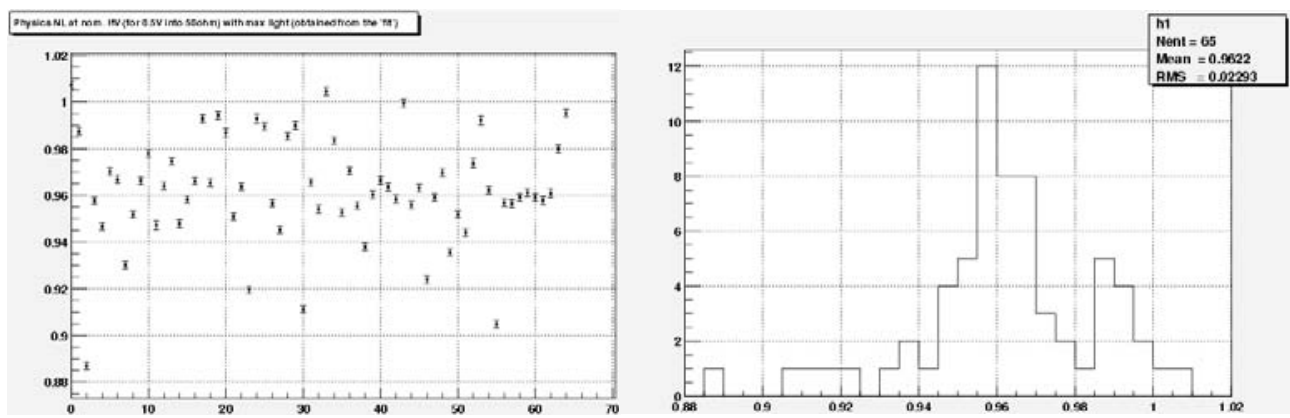

Figure 3.28: (eft) Linearity $\left(\operatorname{lin}_{1}\right)$ of PMTs at expected light levels $\left(L_{\text {exp }}\right)$ vs PMT\#. (right) Distribution of $\operatorname{lin}_{1}$ for all PMTs.

the Moller detector electronics (described below). The overall linearity of the Moller detector including the electronics was studied in situ during the data collection. A conservative estimate places the linearity (with the entire electronics chain) at $99 \pm 1.1 \%[42]$.

\subsection{Moller Detector Electronics}

The Moller detector is expected to receive a flux of roughly twenty million electrons per spill. From this flux, any one detector segment could receive up to one million electrons (due to the radial profile of the Moller electrons). The readout electronics for the Moller detector should therefore have a per-channel resolution better than 200 ppm, an overall detector resolution better than 40 ppm, and should be linear to within $1 \%$.

A diagram of the Moller detector electronics chain is given in Fig. 3.29. Initially, each PMT's output is run across a low-pass filter to prevent reflections. Since, the high voltage ground and the signal ground are the same within the PMT, isolation transformers are connected to every single PMT's signal output to prevent massive 


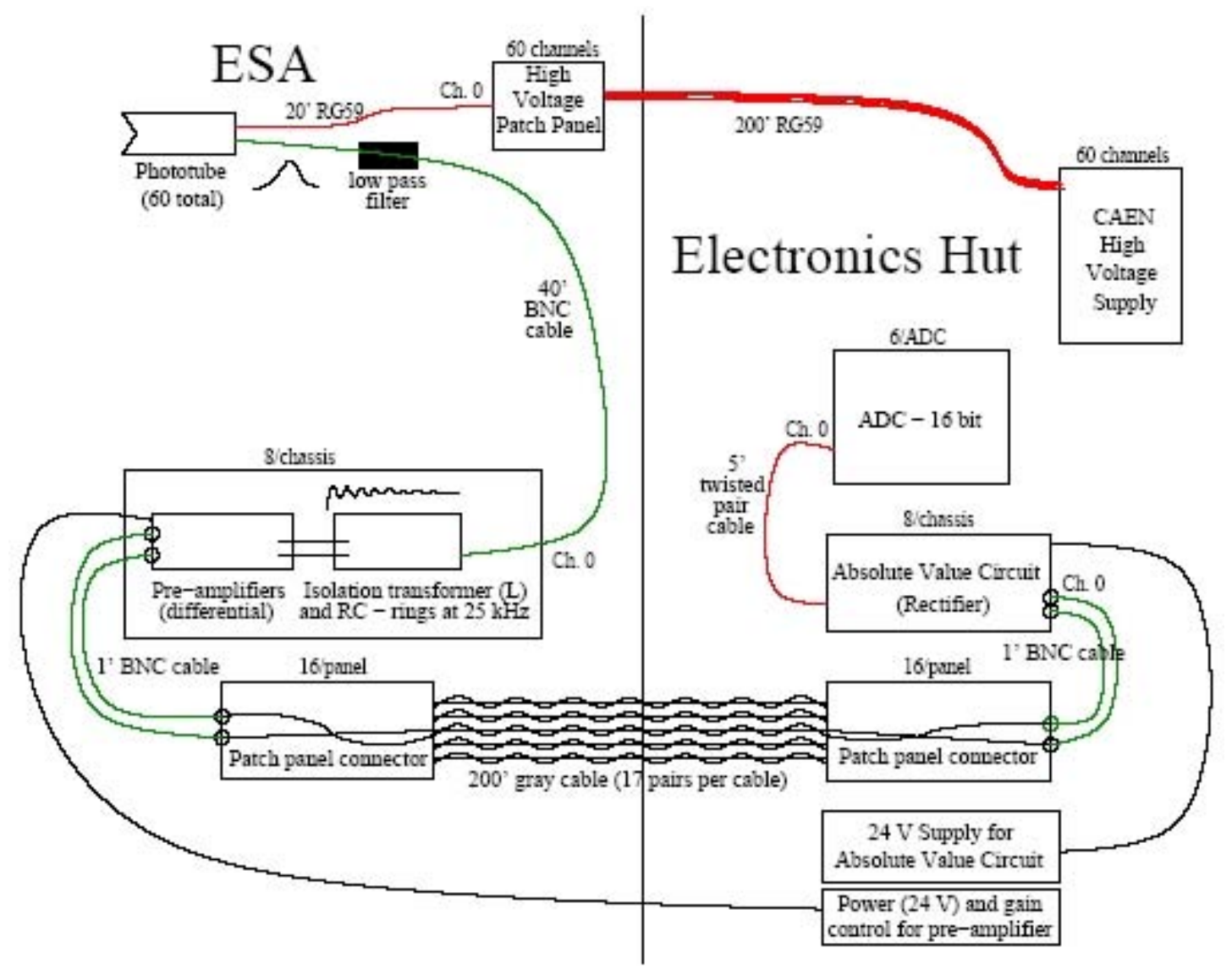

Figure 3.29: Diagram of the Moller detector electronics layout.

ground loops. The low pass filter slows down the PMT output signal, allowing it to deposit all its energy into the isolation transformer. A capacitor and a resistor are added to the isolation transformer in series to create an RLC circuit, which transforms the PMT output into a large ringing signal. Once the ringing signal has been created, it is amplified in two successive gain stages (a 1/2/4/8 and a 10/100). The gain of the amplifier is remotely selectable. The overall circuit is designed to make the ringing signal differential, giving a "free" factor of two in gain while further isolating the chances of noise from ground loops. 
The isolation transformer and the preamplifier are located inside ESA. After the signal has been amplified, it travels down approximately 200 feet of twisted pair cable (to prevent crosstalk) and into an "absolute value" circuit (a rectifier), which is located in a small room outside ESA. Once the signal is rectified, it is run into the integrating ADC described in 2.10.1

To determine the resolution of the Moller detector electronics, the electron beam was run at several different current settings. The square of the width of the experimental asymmetry for each setting was then plotted against $1 / N(N$ is the number of electrons per spill). A fit to this data will produce an offset which should be the square of the detector resolution. Fig. 3.30 shows the results of the scan. Taking the offset from the graph, the detector resolution is $\sqrt{12060}=100 \mathrm{ppm}$. The width of the Moller detector asymmetry is typically around 190-200 ppm,

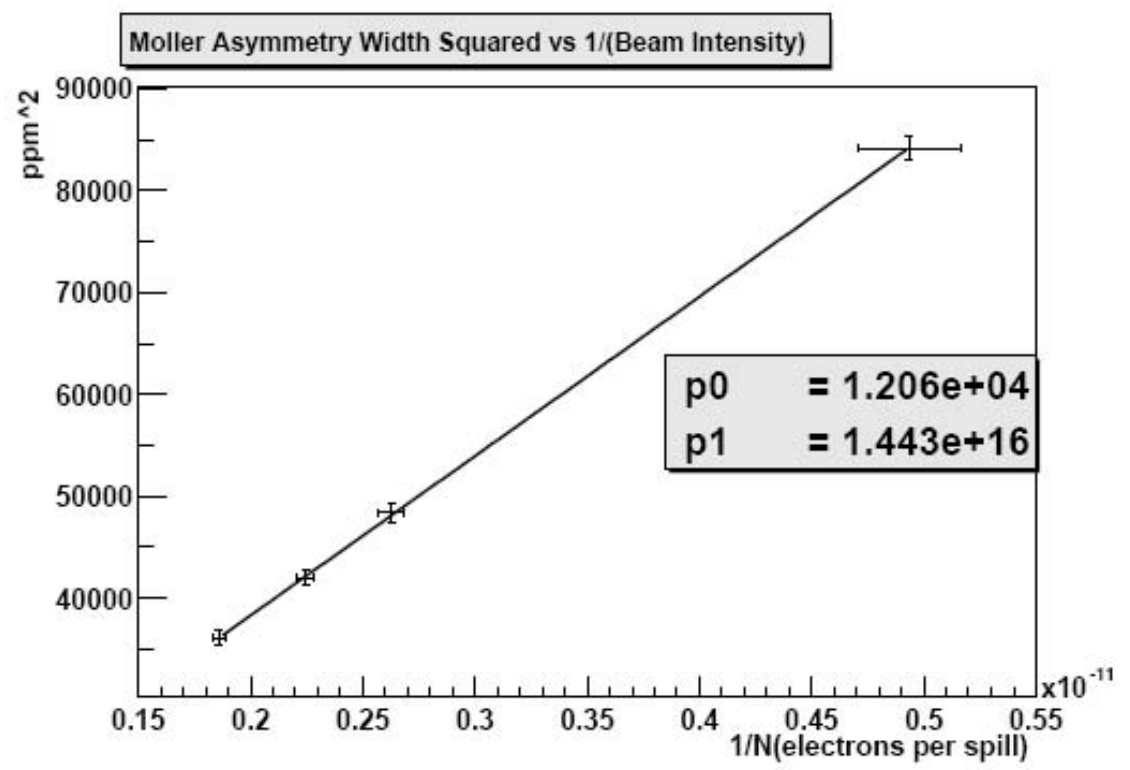

Figure 3.30: A plot of $\sigma_{\text {Moller }}^{2}$ vs 1/(beam intensity) for different beam intensities. The Moller detector resolution is $\sqrt{p 0}=110 \mathrm{ppm}[16]$. 
so the detector electronics' contribution to this width is non-negligible. Possible causes of the $110 \mathrm{ppm}$ resolution include noise from the amplifiers in the first stage of the Moller detector electronics, as well as pedestal noise. 
E158 Calorimeter 


\section{Chapter 4}

\section{Preliminary Results for $A_{P V}$ and $\sin ^{2} \theta_{W}$}

\subsection{Data Processing}

E158 collected data at $120 \mathrm{~Hz}$ or $60 \mathrm{~Hz}$ for most of its physics running. The information was recorded on tapes in files containing typically an hour's worth of data. Such hour long data runs typically contained 400,000 pulses or events (at $120 \mathrm{~Hz}$ ). For the spring 2002 physics data, E158 collected over 250M events corresponding to around $450 \mathrm{~Gb}$ of data. This chapter will include results from spring 2002 data only.

As discussed earlier, the E158 data was taken at two different beam energies (45 GeV and $48 \mathrm{GeV}$ ). During each physics data collection period the beam energy was changed at least once. The physics asymmetry is calculated for the two data sets separately, because the asymmetry corrections and the beam systematics can be different for the two beam energies. Furthermore, the half wave plate state 
described in chapter 2 was flipped roughly once in two days. On the basis of half wave plate state, the whole data is divided into smaller subsets of runs called "slugs". The spring 2002 data consists of 24 slugs.

Several tasks are performed on data before it is used for the asymmetry calculations. The data analysis software, called "the framework", passes through the data several times for processing. In the first pass, the ESA and ASSET data streams are synchronized and merged into single data stream. The events failing synchronization are cut from the data. In the next data pass, the pedestals are calculated for all ADC channels (for all detectors and monitors), and are subtracted from the data. In the third pass, the pedestal-subtracted data are written to a ROOT file.

\subsubsection{Pedestal Subtraction}

The frequency of the pedestal pulses is $0.5 \mathrm{~Hz}$. The pedestal subtraction algorithm starts by calculating the average pedestal value for the first ten pedestal pulses in a data run. This corresponds to first $20 \mathrm{sec}$ of data which is discarded from each run. The average pedestal values are then subtracted from the data until the next pedestal pulse is reached. At that stage, the average over 10 pedestal pulses is recalculated including the latest pedestal pulse. The algorithm repeats itself until the end of the run is reached.

\subsubsection{Data Cuts}

Several data cuts are also applied during the first pass. These cuts can be divided into two classes. The "baseline" cuts remove the pedestal pulses, witness pulses and the events where the beam was "dithered" intentionally. The baseline cuts also removes the events with electronics glitches, DAQ failures and dead or saturated 
ADC channels.

The second set of cuts remove all the events in which the beam parameters are out of the acceptable limits to ensure the quality of the physics data. Most of these cuts are stretched in time using a simple algorithm which finds events failing the cut and removes several hundred or thousand points before and after these events. This decreases the chances of systematic bias affecting the data set. The detail of these cuts can be found in [54]. Once all these cuts are made the analysis of the physics data can begin.

\subsection{Measuring an Asymmetry}

The cross section $\sigma$ is proportional to the scattered flux normalized with the incident beam intensity, i.e., the ratio of the detected scattered flux $S$ (or detector signal) and the incident beam current $Q: \sigma \propto S / Q$. Thus the asymmetry in the cross section is equivalent to the asymmetry in the charge normalized detector signal:

$$
A_{i}^{\circ}=\frac{S_{R_{i}} / Q_{R}-S_{L_{i}} / Q_{L}}{S_{R_{i}} / Q_{R}+S_{L_{i}} / Q_{L}}
$$

where $S_{R_{i}}\left(S_{L_{i}}\right)$ is the signal from the $i$ th channel of the detector for the right (left) handed beam pulse. $Q_{R}\left(Q_{L}\right)$ is the charge in the incident right(left) pulse. $A_{i}^{\circ}$ is the asymmetry per pulse pair.

The asymmetry in 4.2.1 is, at first, regressed against the beam parameters by subtracting a linear component of all monitor asymmetries from each detector channel asymmetry: 


$$
A_{i}^{r e g}=A_{i}^{\circ}-\sum m_{i j} X_{j}
$$

where $X_{j}$ stands for the charge asymmetry $\left(\frac{Q_{R}-Q_{L}}{Q_{R}+Q_{L}}\right)$, and the right/left differences in beam energy $\left(E_{R}-E_{L}\right), x$ - and $y$-positions $\left(x_{R}-x_{L}, y_{R}-y_{L}\right)$, and $x$ - and $y$-angles $\left(d x_{R}-d x_{L}, d y_{R}-d y_{L}\right)$. This should remove the dependence of detector asymmetry on the beam parameters. In principle, the regression slopes $m_{i j}$ can be different for each pulse pair. But, practically, $m_{i j}$ are calculated from 10000 pulse pairs, and hence are constant for those 10000 pulse pairs.

The regressed asymmetry per pulse pair for the whole detector is obtained by the weighted sum of the regressed asymmetries of each channel:

$$
A^{r e g}=\frac{1}{N_{c h}} \frac{\sum_{N_{c h}} A_{i}^{r e g} w_{i}}{\sum_{N_{c h}} w_{i}}
$$

where $N_{c h}$ is the number of channels in the detector. To calculate the weights $w_{i}$, first the covariance matrix of the detector is calculated:

$$
M_{i j}=\frac{1}{N_{p p}} \sum_{N_{p p}} A_{i}^{r e g} A_{j}^{r e g}-\frac{1}{N_{p p}^{2}} \sum_{N_{p p}} A_{i}^{r e g} \sum_{N_{p p}} A_{j}^{r e g}
$$

where $N_{p p}$ is the total number of pulse pairs in one data run. $M_{i j}$ gives the covariance between the channel $i$ and channel $j$ of the detector. The weights $w_{i}$ are obtained by minimizing $\sum_{i, j}\left(w_{i}^{\prime} w_{j}^{\prime} M_{i j}\right)^{1,2}$. The weights $w_{i}^{\prime}$ are given by the width of the regressed asymmetry per run for each channel:

$$
w_{i}^{\prime}=\frac{1}{\sigma^{2}\left(A_{i}^{r e g}\right)}
$$

\footnotetext{
${ }^{1} w_{i}^{\prime} \longrightarrow w_{i}$ for $\min \left[\sum_{i, j} w_{i}^{\prime} w_{j}^{\prime} M_{i j}\right]$

${ }^{2}$ The actual minimization scheme for the Moller detector also takes into account the effects of the transverse polarization component of the beam noticed by the Moller detector. See [54].
} 


$$
\sigma^{2}\left(A_{i}^{r e g}\right)=\frac{1}{N_{p p}} \sum_{N_{p p}}\left(A_{i}^{r e g}-<A_{i}^{r e g}>\right)^{2}
$$

Note that the weights $w_{i}$ are constant for the whole data run (i.e., are the same for all pulse pairs in one run). The Moller detector asymmetry per run $\left(\mathcal{A}_{n}\right)$ is simply the arithmetic average of the regressed asymmetry $\left(A^{\text {reg }}\right)$ over the number of pulse pairs in the run:

$$
\mathcal{A}_{n}=\frac{1}{N_{p p}} \sum_{N_{p p}} A^{r e g}
$$

where the index $n$ stands for the run number. The width and the error of $\mathcal{A}_{n}$ are simply obtained from the distribution of $\mathcal{A}_{n}$ :

$$
\begin{gathered}
\sigma^{2}\left(\mathcal{A}_{n}\right)=\frac{1}{N_{p p}} \sum_{N_{p p}}\left(A^{r e g}-\mathcal{A}_{n}\right)^{2} \\
\delta \mathcal{A}_{n}=\frac{\sigma\left(\mathcal{A}_{n}\right)}{\sqrt{N_{p p}}}
\end{gathered}
$$

Fig. 4.1 shows how the width of raw asymmetry distribution of a Moller channel changes after charge normalization and regression. The above description is somewhat general and can be used for any detector. For the $e P$ detector, the weights are not optimized, rather, the statistical weights (4.2.5) are used for averaging. On the other hand, for the luminosity monitor simple average over the channels is taken.

The grand average of the asymmetry over all data runs $\left(N_{\text {run }}\right)$ for one particular beam energy $E$ is:

$$
\mathcal{A}_{E}=\frac{\sum_{N_{\text {run }}} \mathcal{A}_{n} / \sigma^{2}\left(\mathcal{A}_{n}\right)}{\sum_{N_{\text {run }}} 1 / \sigma^{2}\left(\mathcal{A}_{n}\right)},
$$




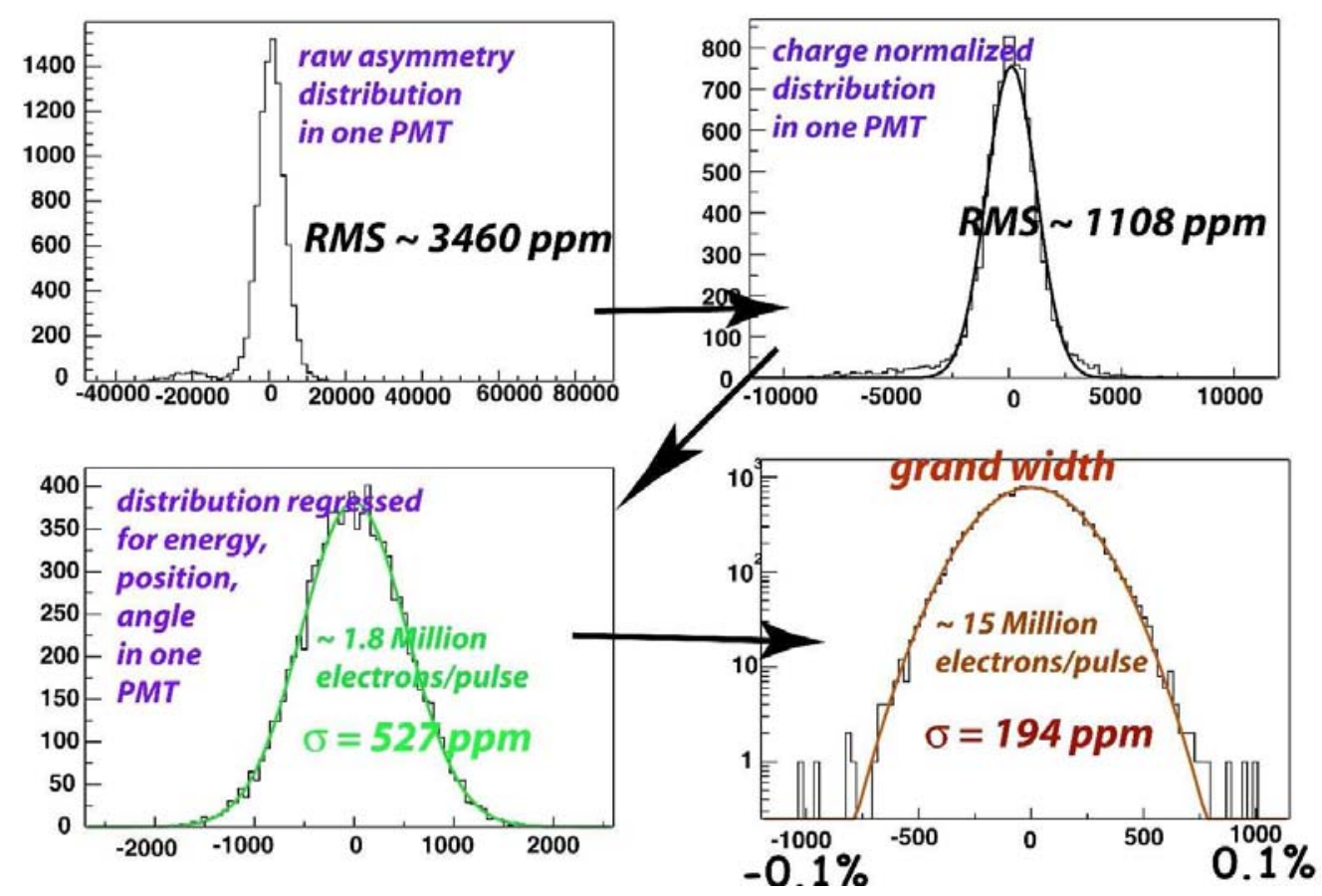

Figure 4.1: Moller asymmetry distribution for one run, starting from the left-right asymmetry in the detected signal from one PMT to average asymmetry of complete Moller detector.

with the error and width given by

$$
\begin{gathered}
\delta \mathcal{A}_{E}=\frac{\sigma\left(\mathcal{A}_{E}\right)}{\sqrt{N_{\text {run }}}} \\
\frac{1}{\sigma^{2}\left(\mathcal{A}_{E}\right)}=\sum_{N_{\text {run }}} \frac{1}{\sigma^{2}\left(\mathcal{A}_{n}\right)}
\end{gathered}
$$

Note that $N_{\text {run }}$ can be different for the two data sets for two beam energies $(45 \mathrm{GeV}$ and $48 \mathrm{GeV})$. 


\subsection{Moller Detector Asymmetry}

Fig. 4.2 shows the Moller asymmetry vs the slug number. (Here $\mathcal{A}_{n}$ is averaged over the runs in each slug.) The $\chi^{2} /$ ndf of a simple linear fit to the data is close to one. It is clear from the plot that the asymmetry is quite stable over much longer time scale. To see the shape of the distribution around the mean, one can look at the pull plot. Fig. 4.3(a) shows the pull plot per run, which is a histogram of $\frac{\mathcal{A}_{n}-<\mathcal{A}_{n}>}{\sigma\left(\mathcal{A}_{n}\right)}$, and Fig. 4.3(b) shows the pull plot per pulse pair $\left(\frac{A^{r e g}-<\mathcal{A}_{n}>}{\sigma\left(A^{r e g}\right)}\right)$. Both of these plots have gaussian shapes, and no significant outliers are present. From these plots it is clear that no significant systematics are affecting the data.

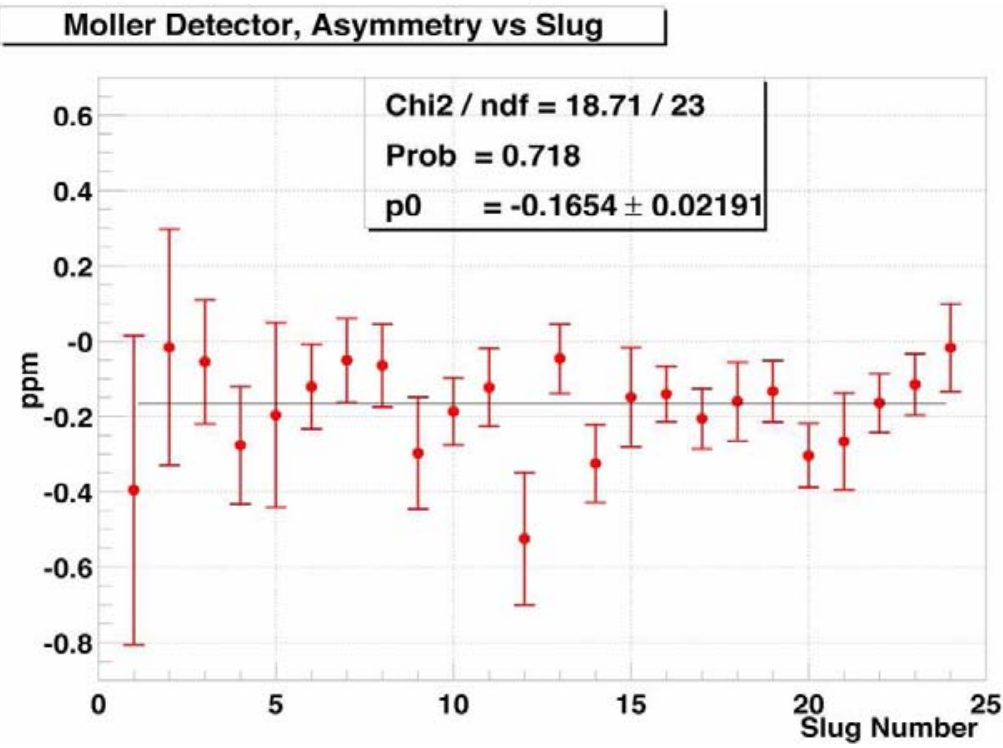

Figure 4.2: Moller detector asymmetry vs slug number.

The change in the beam energy and the half wave plate state practically flips the sign of the asymmetry $\left(\mathcal{A}_{n}\right)$. In principle, the systematic contribution to the asymmetry should remain the same for all these states. Fig. 4.4(a) and (b) show the comparison of the asymmetries for different sign flips. It is evident that the absolute value of the 


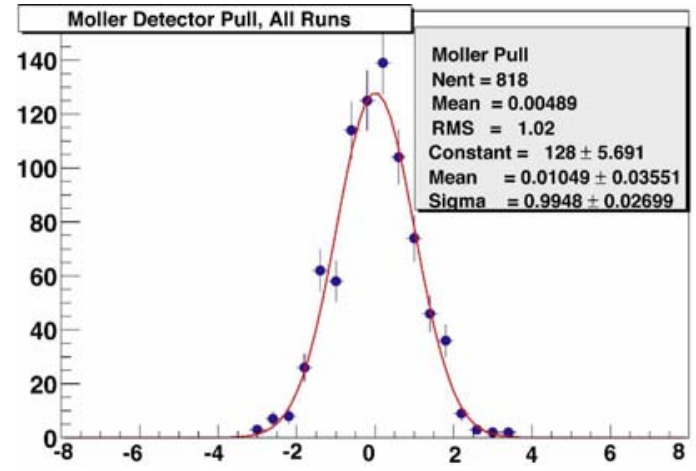

(a) $\frac{\mathcal{A}_{n}-<\mathcal{A}_{n}>}{\sigma\left(\mathcal{A}_{n}\right)}$

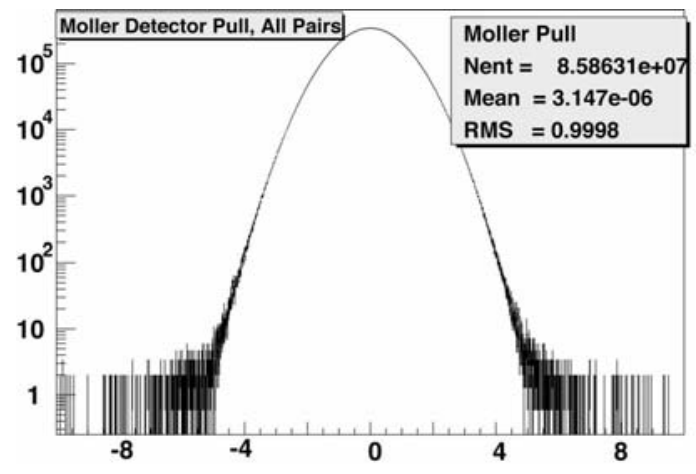

(b) $\frac{A^{r e g}-<\mathcal{A}_{n}>}{\sigma\left(A^{r e g}\right)}$

Figure 4.3: Moller detector asymmetry pull plots.

asymmetry does not change significantly for any sign flip. All these plots clearly bound the systematic error to at least the level of the statistical error.

\section{Moller Detector, Asymmetry vs Slug}

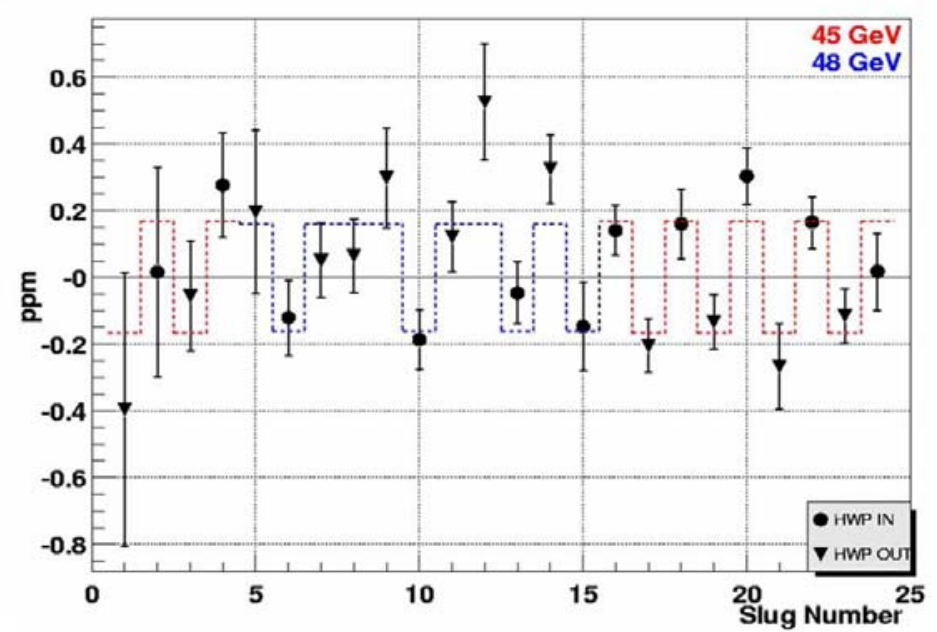

Moller Detector Asymmetry

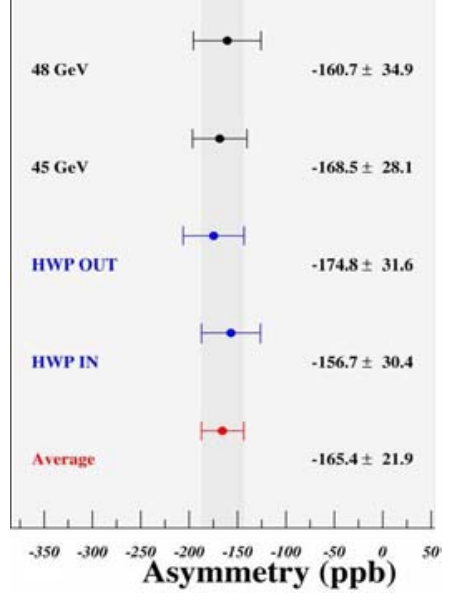

Figure 4.4: (left) Moller detector asymmetry for all slugs. The sign of the asymmetry is not corrected for different energy and half wave plate states. (right) Average asymmetry for each energy and half wave plate state. 


\section{$4.4 \quad e P$ Asymmetry}

Fig. 4.5 shows the plot of the $e P$ asymmetry vs slug number, in which the sign of the asymmetry is not corrected for the sign of the half wave plate state and the energy state. The parity violating asymmetry is proportional to $Q^{2}$. For inelastic $e P, A_{P V} \sim 10^{-4} Q^{2}$, and for elastic $e P, A_{P V} \sim 10^{-5} Q^{2}$ at low $Q^{2}$. Since the $Q^{2}$ of the spectrometer for the $e P$ is significantly different for the two energy states, the $e P$ detector exhibits two different asymmetries. The two values are $-1.430 \pm 0.045 \mathrm{ppm}$ for $45 \mathrm{GeV}$, and $-1.735 \pm 0.063 \mathrm{ppm}$ for $48 \mathrm{GeV}$.

The $e P$ systematic correction to the Moller detector asymmetry was determined using the Monte Carlo simulation created specifically for E158 [55]. This Monte Carlo simulation was perfected using the profile detector data. The profile detector was used during the experiment to map out the distribution of the Moller and $e P$ scatters, both

\section{ep Detector, Asymmetry vs Slug}

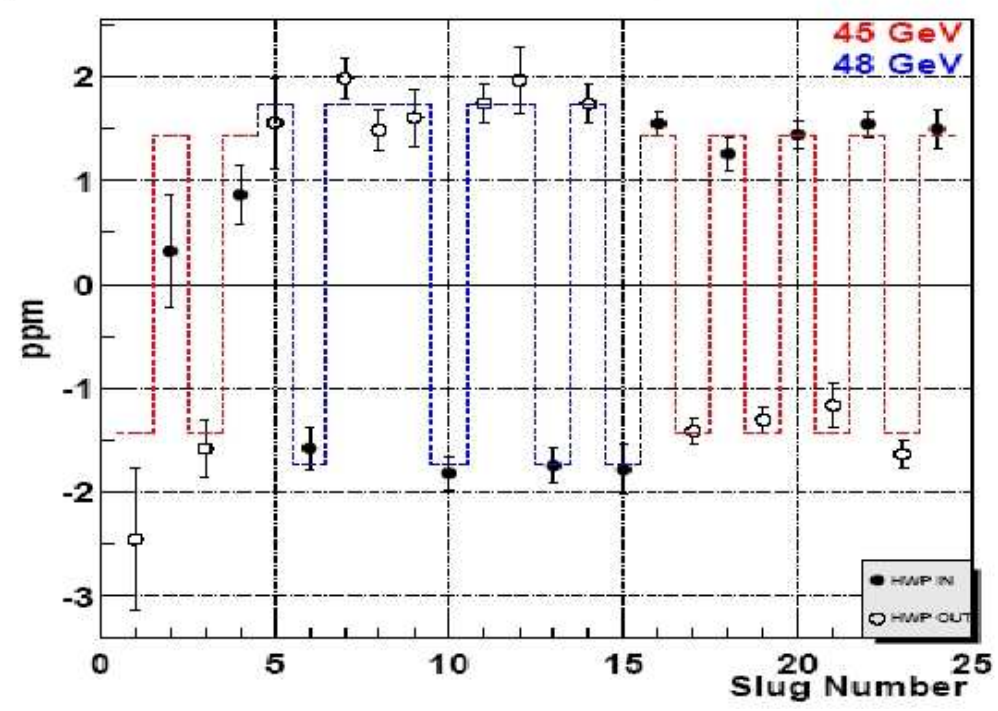

Figure 4.5: $e P$ detector asymmetry, the sign of the asymmetry is not corrected for the half wave plate state and energy state. 
with and without the holey collimator in position. With the holey collimator in position, a large separation is observed between the two distributions, which allows to measure the amount of $e P$ scatters between the two peaks. Once the Monte Carlo simulation is adjusted to provide a reasonable simulation of the Moller and $e P$ kinematics, it can be used to determine the amount of $e P$ flux in the Moller detector. Fig. 4.6 shows the comparison between the Monte Carlo simulation and profile detector data, both with and without the holey collimator.

The $e P$ background (as well as all other backgrounds) increases the observed flux of the Moller electrons $\left(S_{R}+S_{L}\right)$. This, in turn, decreases the asymmetry, $A_{P V}=\frac{S_{R}-S_{L}}{S_{R}+S_{L}}$. Thus, beside making a shift to the experimental asymmetry, the $e P$ background also leads to a dilution of (decrease in) the asymmetry. The level of this dilution and the asymmetry corrections are given in Table 4.1.

\begin{tabular}{|l|c|c|}
\hline & $45 \mathrm{GeV}$ & $48 \mathrm{GeV}$ \\
\hline \hline$e P$ elastic correction & $-10.0 \pm 2.8 \mathrm{ppb}$ & $-13.0 \pm 3.1 \mathrm{ppb}$ \\
\hline$e P$ elastic dilution & $0.0758 \pm 0.0042$ & $0.0791 \pm 0.0047$ \\
\hline$e P$ inelastic correction & $-30.7 \pm 7.4 \mathrm{ppb}$ & $-40.2 \pm 9.6 \mathrm{ppb}$ \\
\hline$e P$ inelastic dilution & $0.0138 \pm 0.0034$ & $0.0163 \pm 0.0041$ \\
\hline
\end{tabular}

Table 4.1: The dilutions and corrections to the Moller asymmetry from the elastic and inelastic $e P$ backgrounds.

\subsection{Physics Asymmetry}

The physics asymmetry for each beam energy is obtained by subtracting the background and beam asymmetries from $\mathcal{A}_{E}$ and dividing by the scale factors and dilution factors:

$$
\mathbf{A}_{E}=\frac{\mathcal{A}_{E}-d \mathcal{A}_{E}}{S\left(1-f_{E}\right)}
$$



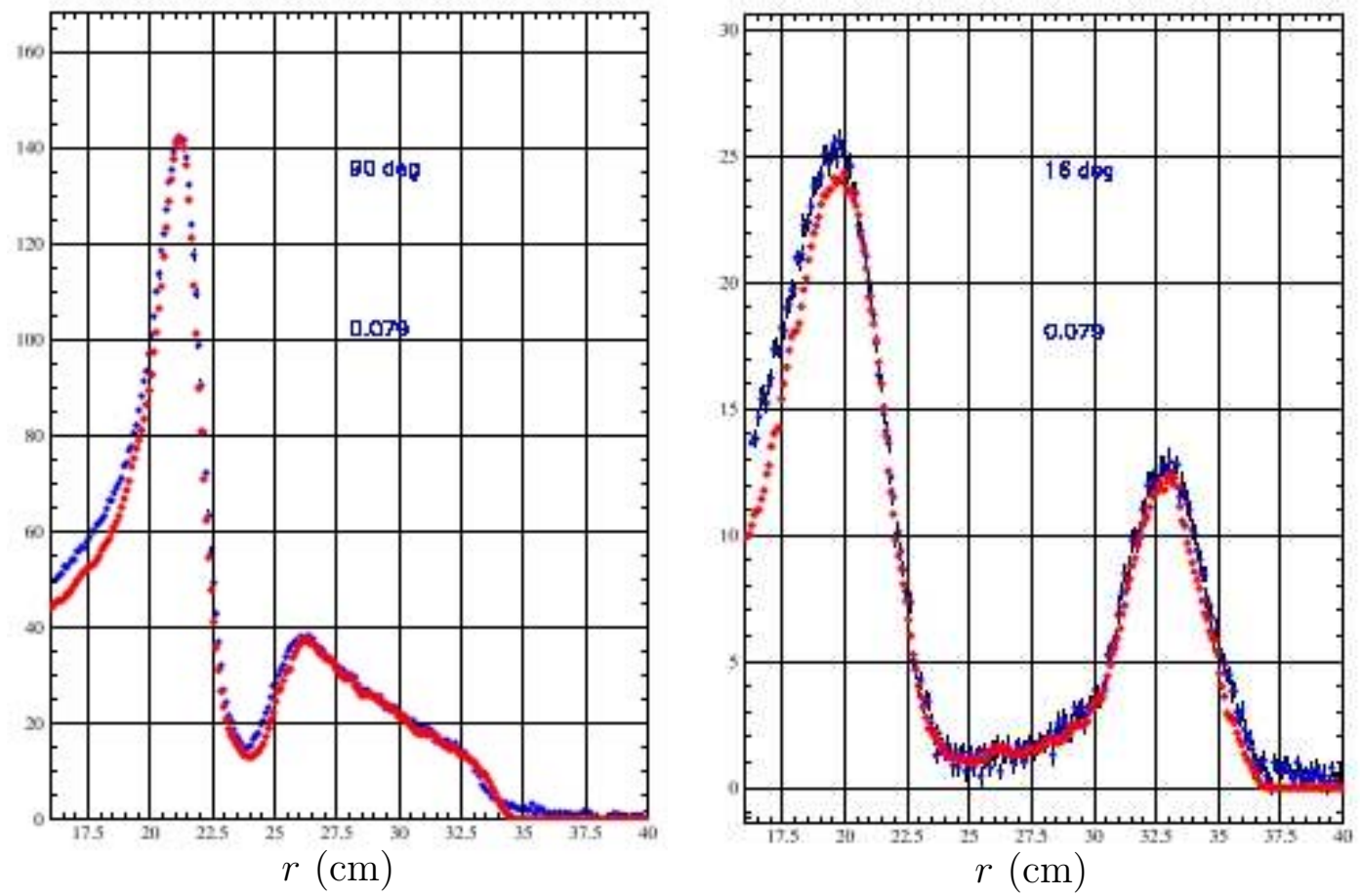

Figure 4.6: Comparison between the profile detector data (blue) and Monte Carlo simulation (red) with (right) and without (left) holey collimator.

where $d \mathcal{A}_{E} \pm \delta\left(d \mathcal{A}_{E}\right)$ is total asymmetry correction due to beam asymmetries, and elastic and inelastic $e P$ asymmetries. The dilution factors $f_{E} \pm \delta f_{E}$ include the dilutions from the $e P$, pion and photons etc, and are different for different energies. The scale factor $S \pm \delta S$ is the sum of the detector linearity $(0.990 \pm 0.011)$ and beam polarization $(0.850 \pm 0.050)$.

Tables 4.2 and 4.3 give the list of the asymmetry corrections and the dilution factors for the spring 2002 data. At $45 \mathrm{GeV}, 4.5 .1$ gives an asymmetry of $-162.4 \pm 37.2$ (stat) \pm 30.6 (syst) $\mathrm{ppb}$, and at $48 \mathrm{GeV},-136.3 \pm 46.5$ (stat) \pm 31.5 (syst) ppb. The statistical and systematic errors on $\mathbf{A}_{E}$ are obtained by 


$$
\begin{aligned}
\delta \mathbf{A}_{E}(\text { stat }) & =\frac{\delta \mathcal{A}_{E}}{S\left(1-f_{E}\right)} \\
\delta \mathbf{A}_{E}(\text { syst }) & =\left\{\left(\frac{\delta\left(d \mathcal{A}_{E}\right)}{S\left(1-f_{E}\right)}\right)^{2}+\mathbf{A}_{E}^{2}\left(\frac{\delta f_{E}}{1-f_{E}}\right)^{2}+\delta S^{2}\right\}^{1 / 2}
\end{aligned}
$$

The final physics asymmetry is obtained by combining the asymmetries for two energies. The statistical errors are used to weight the two results.

$$
\begin{gathered}
\mathbf{A}=\sum_{E} W_{E} \mathbf{A}_{E} \\
W_{E}=\frac{1 / \delta \mathbf{A}_{E}^{2}}{1 / \delta \mathbf{A}_{E=45}^{2}+1 / \delta \mathbf{A}_{E=48}^{2}}
\end{gathered}
$$

And the statistical error on asymmetry is

$$
\delta \mathbf{A}(\text { stat })=\frac{1}{\sqrt{1 / \delta \mathbf{A}_{E=45}^{2}+1 / \delta \mathbf{A}_{E=48}^{2}}}
$$

\begin{tabular}{|l|c|c|c|c|}
\hline Corrections $(\mathrm{ppb})$ & \multicolumn{2}{|c|}{$45 \mathrm{GeV}$} & \multicolumn{2}{c|}{$48 \mathrm{GeV}$} \\
\hline & $d \mathcal{A}_{E}$ & $\delta\left(d \mathcal{A}_{E}\right)$ & $d \mathcal{A}_{E}$ & $\delta\left(d \mathcal{A}_{E}\right)$ \\
\hline \hline Beam Asymmetry & 0 & 0.0183 & 0 & 0.0183 \\
\hline$e P$ elastic & -0.0100 & 0.0028 & -0.0130 & 0.0031 \\
\hline$e P$ inelastic & -0.0307 & 0.0074 & -0.0402 & 0.0096 \\
\hline Spotsize & 0 & 0.005 & 0 & 0.005 \\
\hline Blinded $e P$ & -0.005 & 0.003 & -0.005 & 0.003 \\
\hline Quads off Al asymmetry & 0 & 0.006 & 0 & 0.006 \\
\hline Run $2 P$ asymmetry & 0 & 0.007 & 0 & 0.007 \\
\hline Pions & 0 & 0.005 & 0 & 0.005 \\
\hline \hline Total & -0.0457 & 0.0233 & -0.0583 & 0.0241 \\
\hline
\end{tabular}

Table 4.2: Summary of the corrections to the Moller detector asymmetries. 


\begin{tabular}{|l|c|c|c|c|}
\hline Dilutions & \multicolumn{2}{|c|}{$45 \mathrm{GeV}$} & \multicolumn{2}{c|}{$48 \mathrm{GeV}$} \\
\hline & $f_{E}$ & $\delta f_{E}$ & $f_{E}$ & $\delta f_{E}$ \\
\hline \hline$e P$ elastic & 0.0758 & 0.0042 & 0.0791 & 0.0047 \\
\hline$e P$ inelastic & 0.0138 & 0.0034 & 0.0163 & 0.0041 \\
\hline Photons & 0.0040 & 0.0040 & 0.0040 & 0.0040 \\
\hline Blinded $e P$ & 0.0030 & 0.0010 & 0.0030 & 0.0010 \\
\hline Quads off Al asymmetry & 0.0010 & 0 & 0.0010 & 0 \\
\hline Pions & 0.0020 & 0.0020 & 0.0020 & 0.0020 \\
\hline \hline Total & 0.0995 & 0.0071 & 0.1054 & 0.0077 \\
\hline
\end{tabular}

Table 4.3: Summary of the dilution factors for the Moller detector asymmetries.

To calculate the systematic error, we first calculate the errors on the corrections and dilutions for both energies:

$$
\begin{gathered}
\delta(d \mathcal{A})=\sum_{E} \frac{W_{E} \delta\left(d \mathcal{A}_{E}\right)}{S\left(1-f_{E}\right)} \\
\delta f=\sum_{E} W_{E} \mathbf{A}_{E} \frac{\delta f_{E}}{1-f_{E}}
\end{gathered}
$$

The systematic error on the final physics asymmetry is

$$
\delta \mathbf{A}(\text { syst })=\sqrt{\delta(d \mathcal{A})^{2}+\delta f^{2}+(\mathbf{A} \delta S / S)^{2}}
$$

The final parity violating asymmetry in the Moller scattering at a $Q^{2}$ of $0.027 \mathrm{GeV}^{2}$ is $-151.9 \pm 29.0($ stat $) \pm 32.5$ (syst) $\mathrm{ppb}$ (for the spring 2002 data).

\subsection{Calculation of $\sin ^{2} \theta_{W}$}

The $\sin ^{2} \theta_{W}$ can be calculated by using the value of $\mathbf{A}_{E}$ in 1.4.3. Rewriting equation 1.4.3 for $\sin ^{2} \theta_{W}$ we can have 


$$
\left(\sin ^{2} \theta_{W}\right)_{E}=\frac{1}{4}+\frac{\mathbf{A}_{E}}{P_{E}}
$$

where

$$
P_{E}=-m_{e} E_{\text {beam }} \frac{G_{F}}{\sqrt{2} \pi \alpha} \frac{16 \sin ^{2} \theta_{c m}}{\left(3+\cos ^{2} \theta_{c m}\right)^{2}}
$$

$P_{E}$ is called the analyzing power and is determined using the Monte Carlo simulation. For $45 \mathrm{GeV}, P_{E}=12.936 \pm 0.010$ and for $48 \mathrm{GeV}, P_{E}=13.221 \pm 0.010$. These values of $P_{E}$ need to be corrected for the bremsstrahlung radiation because the GEANT simulation used to determine $P_{E}$ does not handle the bremsstrahlung radiation properly. As determined in [56], these corrections can be made by simply multiplying $P_{E}$ by the factor $B_{r}=0.900 \pm 0.009$. Substituting the values of $\mathbf{A}_{E}$ and the corrected values of $P_{E}$ in 4.6.1, we get the $\sin ^{2} \theta_{W}$ for both energies:

$$
\begin{aligned}
& \left(\sin ^{2} \theta_{W}\right)_{E=45 \mathrm{GeV}}=0.2361 \pm 0.0032(\text { stat }) \pm 0.0027(\text { syst }) \\
& \left(\sin ^{2} \theta_{W}\right)_{E=48 \mathrm{GeV}}=0.2371 \pm 0.0039(\text { stat }) \pm 0.0028(\text { syst })
\end{aligned}
$$

The above results are combined by adding the values weighted by the statistical errors. Thus, the final value of $\sin ^{2} \theta_{W}$ at a $Q^{2}=0.0027$ is:

$$
\sin ^{2} \theta_{W}=0.2371 \pm 0.0025(\text { stat }) \pm 0.0027 \text { (syst) }
$$

\subsection{Summary}

E158 has observed for the first time the parity violation in Moller scattering. The preliminary result for the parity violating asymmetry measured by E158 is $-151.9 \pm$ 
29.0 (stat) \pm 32.5 (syst) at a $Q^{2}$ of $0.027 \mathrm{GeV}^{2}$. This is the smallest asymmetry ever measured in a parity violating electron scattering experiment. In addition to measuring the asymmetry in Moller scattering, E158 has also made the most precise measurement of a parity violating asymmetry in the electron-proton scattering.

The value of $\sin ^{2} \theta_{W}$ determined by E158 is $0.2371 \pm 0.0025$ (stat) \pm 0.0027 (syst). The standard model prediction of $\sin ^{2} \theta_{W}$ at a $Q^{2}$ of $0.027 \mathrm{GeV}^{2}$ is $0.2386 \pm 0.0006$ [15]. The difference between the predicted value and the measured value of $\sin ^{2} \theta_{W}$ is $0.0017 \pm 0.0037$, which is less than one sigma. To compare the E158 results with other experiments we convert the above value of $\sin ^{2} \theta_{W}$ to $\sin ^{2} \theta_{W}\left(m_{Z}\right) \overline{M S}=0.2296 \pm 0.0038$. Fig.4.7 show where the current E158 result stands. These results are based on the data collected during the spring 2002 physics run. The addition of the data from the two later physics data sets will reduce the error on $\sin ^{2} \theta_{W}$ to 0.001 .
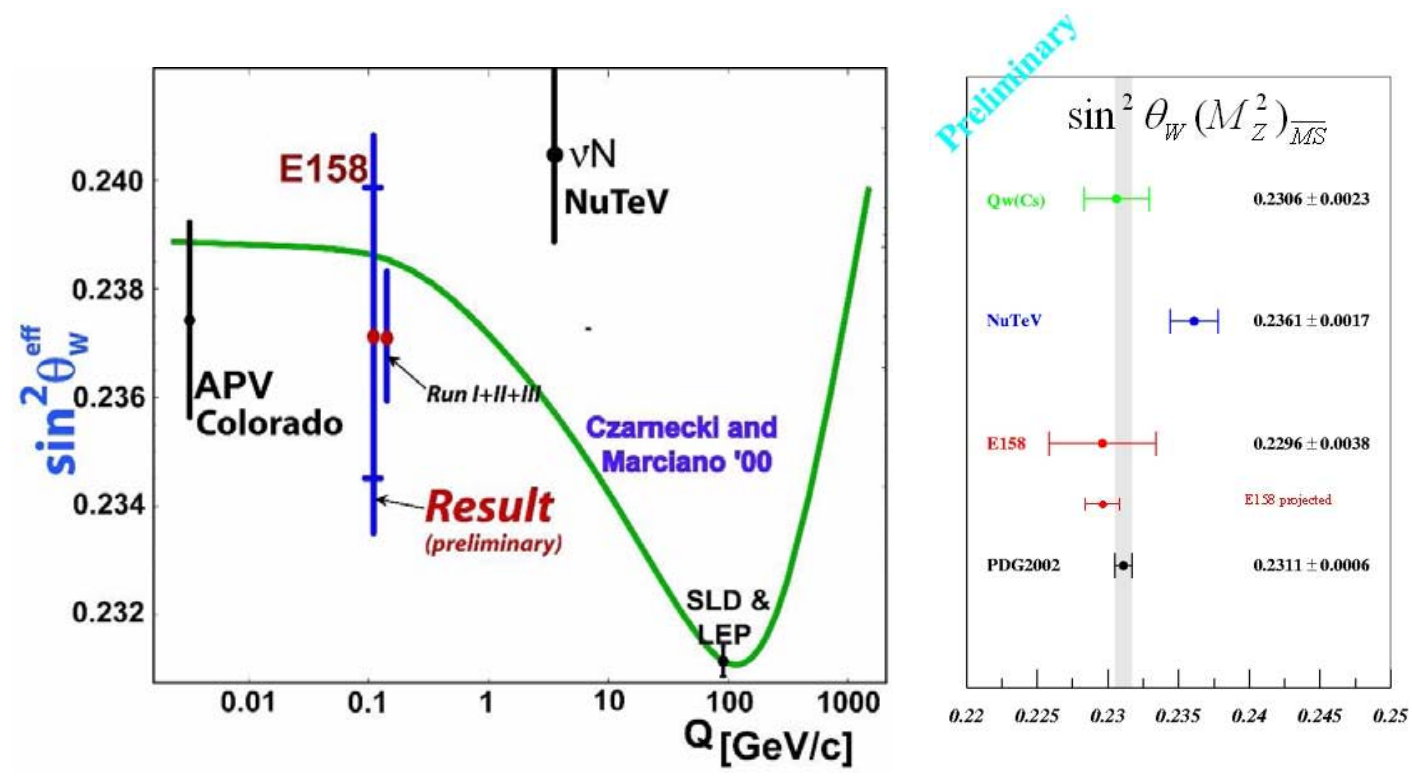

Figure 4.7: Comparison of E158 results with the standard model prediction (left) and other experiments (right). 


\section{Appendix A}

\section{First Results from E158}

The following paper has been submitted to the procedings of 5th International Workshop on Neutrino Factories and Superbeams (NuFact03), Columbia University, New York, 511 June 2003. 



\title{
First Results from E158 Measuring Parity Violation in Moller Scattering
}

\author{
Imran Younus $^{1}$ \\ Department of Physics, Syracuse University, Syracuse, NY 13244
}

\begin{abstract}
SLAC E158 is an experiment to measure the parity nonconserving asymmetry in Moller scattering. $48 \mathrm{GeV}$ longitudinally polarized electrons are scattered off unpolarized (atomic) electrons in a liquid hydrogen target at an average $Q^{2}$ of $0.027 \mathrm{GeV}^{2}$. The asymmetry in this process is proportional to $\left(\frac{1}{4}-\sin ^{2} \theta_{W}\right)$, where $\sin ^{2} \theta_{W}$ gives the weak mixing angle. The preliminary results give $A_{P V}=-151.9 \pm 29.0$ (stat $) \pm 32.5$ (syst) parts per billion. This in tern gives $\sin ^{2} \theta_{W}^{\text {eff }}=0.2371 \pm 0.0025 \pm 0.0027$ which is consistant with the standard model prediction $(0.2386 \pm 0.0006)$.
\end{abstract}

\section{INTRODUCTION}

Over the last three decades, the Standard Model has evolved as the most successful theory describing the electroweak interactions, and has been verified experimentally with an ever-increasing accuracy. The experiments studying the weak interactions at high energies (at $Z^{\circ}$ resonance), have measured the electroweak parameters, such as $\sin ^{2} \theta_{W}$, with spectacular precision, establishing the "correctness" of the Standard Model. However, at lower energies, tests of the electroweak theory in the weak neutral current sector are less sensitive by more than an order of magnitude. Equally precise measurements off the $Z^{\circ}$ resonance are crutial for the "completeness", and can provide an insight to the new physics beyond the standard model.

The SLAC E158 experiment $[1,2]$ is measuring the weak mixing angle at low $Q^{2}$ by observing the parity-violating asymmetries $\left(A_{P V}\right)$ in polarized Moller scattering $\left(e^{-} e^{-} \rightarrow e^{-} e^{-}\right)$. This asymmetry arises from the interference between the electromagnetic and weak neutral currents, and has not been measured before. The goal of the experiment is to measure the asymmetry with a precision of $8 \%$ which would in tern give the most precise measurement to date of $\sin ^{2} \theta_{W}$ at low $Q^{2}$ with $\sigma<0.1 \%$. Such measurement of $\sin ^{2} \theta_{W}$, when compared with the measurements at $Z^{\circ}$ pole, would establish the $Q^{2}$ dependance of the weak coupling angle at a singnificance of $\sim 8 \sigma$.

The E158 results are sensitive to several new physics scenarios at TeV scale. A deviation from the prediction of $\sin ^{2} \theta_{W}$ can indicate the presence of additional neutral current effects such as $Z^{\prime}$ bosons in the mass range of 600 to $900 \mathrm{GeV}$. E158 is also capable of setting a limit to the electron compositness scale of up to 10 to $14 \mathrm{TeV}$, thus providing a complementary approach to high-energy collider experiments' searches.

\footnotetext{
${ }^{1}$ Speaker. For the SLAC E158 Collaboration.
} 


\section{PARITY VIOLATING MOLLER SCATTERING}

The Moller scattering proceeds via both $t$ - and $u$-channel processes. The parity-violating asymmetry in scattering of polarized electrons off unpolarized target is defined as

$$
A_{P V}=\frac{\sigma_{R}-\sigma_{L}}{\sigma_{R}+\sigma_{L}}
$$

where $\sigma_{R}\left(\sigma_{L}\right)$ is the scattering cross-section from incident right(left) handed electrons. The interference between the tree level $\gamma$ and $Z^{\circ}$ amplitudes gives rise to the standard model asymmetry given by[3]

$$
A_{P V}=-m_{e} E \frac{G_{F}}{\sqrt{2} \pi \alpha} \frac{4 \sin ^{2} \theta_{c m}}{\left(3+\cos ^{2} \theta_{c m}\right)^{2}}\left(1-4 \sin ^{2} \theta_{W}\right) .
$$

The simple functional form of the asymmetry suggests Moller scattering to be an excellent probe of the weak mixing angle. At the tree level, for $Q^{2} \approx 0.03 \mathrm{GeV}^{2}$, the asymmetry $A_{P V} \approx-280 \times 10^{-9}$. The electroweak radiative corrections decrease this asymmetry by almost $40 \%[4]$. The beam polarization of $80 \%$ and some dilution from the background processes bring the raw experimental asymmetry down to approximately 130 parts per billion. The experiment goal is to measure this small asymmetry with a precision of $8 \%$, which would determine the $\sin ^{2} \theta_{W}$ at low $Q^{2}$ with $\sigma\left(\sin ^{2} \theta_{W}\right)<0.001$.

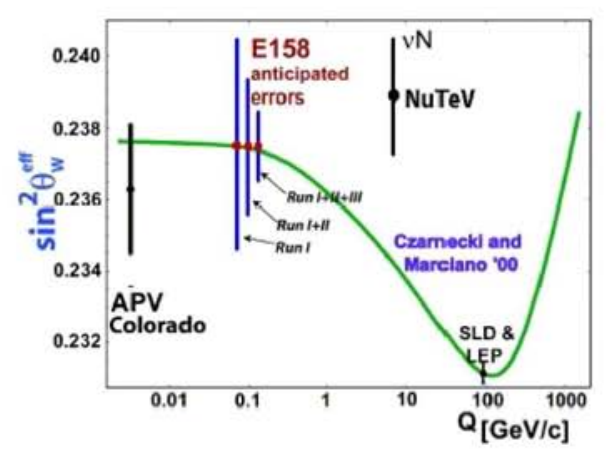

FIGURE 1. Predicted running of $\sin ^{2} \theta_{W}$ from the precision measurement at $Z^{\circ}$ resonance.

The $40 \%$ reduction in the $A_{P V}$ due to the radiative corrections corresponds to a $3 \%$ increase in $\sin ^{2} \theta_{W}$ from its value obtained at $Q^{2}=M_{Z}^{2}$ in measurements obtained by SLC and LEP[5]. This $Q^{2}$ dependance of $\sin ^{2} \theta_{W}$ is shown with solid curve in fig. 1[6].

\section{DESCRIPTION OF THE EXPERIMENT}

High intensity longitudinaly polarized electron beam $\left(3.5-6 \times 10^{11}\right.$ elec./pulse; $300 \mathrm{~ns}$ pulses at $120 \mathrm{~Hz}$ ) is scattered off atomic electrons in an unpolarized liquid hydrogen target. The helicity of the electron beam is flipped psudo-randomly on a pulse-by-pulse basis, and the pulse pair flux asymmetry is measured:

$$
A_{\text {measured }}=\left(F_{R}-F_{L}\right) /\left(F_{R}+F_{L}\right),
$$

where $F_{R}\left(F_{L}\right)$ is the flux of scattered right(left) handed electrons. This asymmetry is then corrected for the differences between the right and left handed beam properties: $A_{\text {raw }}=A_{\text {measured }}-\sum \alpha_{i} \Delta x_{i}$, where $\Delta x_{i}$ stands for the charge, energy, position and angle differences between the right and left handed pulses. The correlation coefficients $\alpha_{i}$ are determined experimentally. The final physics asymmetry is obtained by subtracting 
the backgrounds and normalizing with beam polarization. The background processes mainly consist of elastic and inelestic electron-proton scattering, real and virtual photoproduction of pions and synchrotron radiation.

\section{Apparatus}

E158 uses $150 \mathrm{~cm}$ long liquid hydrogen target ( $0.15 \mathrm{r}$.1.) kept at $17.5 \mathrm{~K}$. The pulse-bypulse density fluctuations in the target are maintained at a level of 65 parts per million. The scattered electrons are transported through a spectrometer consisting of 3 dipole and 4 quadrupole magnets, and an acceptance collimator (several other collimators are used to suppress different backgrounds). The dipole chicane (figure 2) is placed downstream of the target for suppression of soft particles created in the target. The acceptance collimator passes the Moller electrons with momenta in the range $13-25 \mathrm{GeV}$ and $e P$ electrons with momenta of $\sim 40 \mathrm{GeV}$. The quadrupole magnets downstream of the collimator then seperates the Moller and $e P$ fluxes by focusing the Moller electrons on a smaller ring $60 \mathrm{~m}$ downstream of the target where the detector is placed.

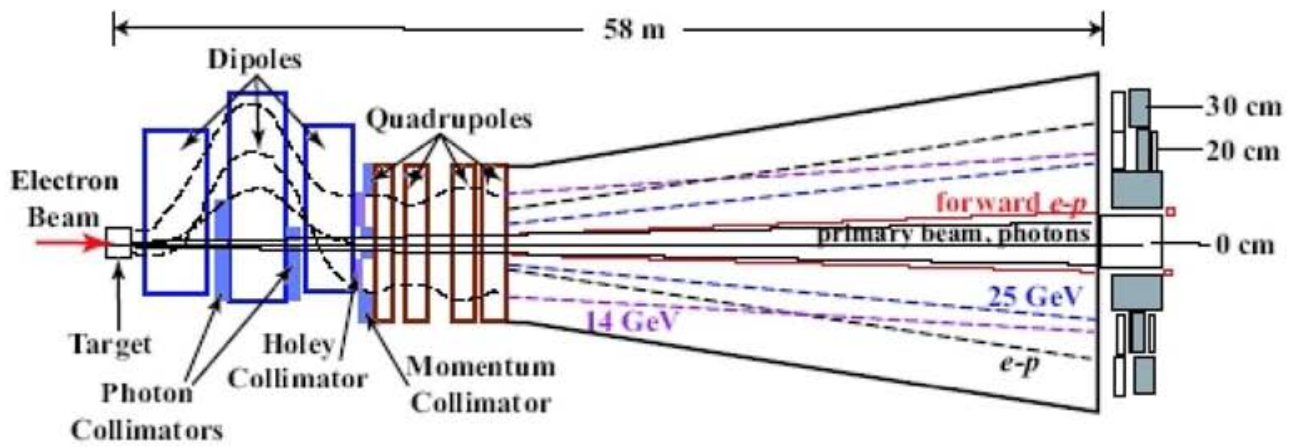

FIGURE 2. Schematic of E158 Spectrometer.

The electromagnetic calorimeter surrounds the beam axis in the radii range 15.0$23.5 \mathrm{~cm}$ and has an energy resolution of $\sim 10 \%$. It consists of layers of quartz fibers interspersed between copper plates, for a total thickness of 15 r.l. The fibers make an angle of $45^{\circ}$ with the beam axis to maximize the Cerenkov light output. The response of the detector is integrated over the duration of the pulse to determine the flux of scattered Moller electrons $\left(3-4.5 \times 10^{7} e^{-} /\right.$pulse $)$.

To measure the elastic and inelestic $e P$ backgrounds, a calorimeter is placed at a large distace from the beam axis surrounding the Moller detecor. The $e P$ detecor is similar in construction to that of the Moller detector but have 10 times lesser quartz density. Additionaly, a pion detecor is placed behind the Moller calorimeter to measure the pion contribution to the Moller signal.

In addition to the above mentioned detectors, a profile detector is placed in front of the main calorimeter to scan the radial and azimuthal distribution of the flux at the face of the Moller detector. It consists of 4 quartz Cerenckov detectors with PMT readouts, which can be remotely positioned at any $r$ and $\phi$. This scanner is used for collimator alignments, background determination, and $Q^{2}$ measurement etc. 


\section{Beam Systematics}

A critical requirement of the experiment is to keep the beam related systematics at the level of few parts per billion. The rapid, psudo-random flipping of the beam helicity on a pulse to pulse basis minimizes the effects of slow drifts in spectrometer acceptance, pedestals or calibrations. However, the pulse to pulse differences in the beam characteristics (intensity, energy, position and angle) can induce false asymmetries. These effects are corrected for by precisely measuring the beam intensity and positions for every pulse, and by applying fast feedbacks. Under these active feedbacks, the false beam asymmetries average out to zero faster than $1 / \sqrt{N}$. Additional cancellation of systematic effects come from the passive reversal of beam helicity. This is done by running the beam at two different energies, 45 and $48 \mathrm{GeV}$ which correspond to integer number of $\mathrm{g}-2$ precessions of electron spin in the $24.5^{\circ}$ bending of the beam before it enters the experimental area.

\section{PRELIMINARY RESULTS}

E158 has observed for the first time the parity violation in the Moller scattering. The preliminary results give $A_{P V}=-151.9 \pm 29.0$ (stat) \pm 32.5 (syst) parts per billion. With these results, the limit on $Z^{\prime}$ mass can be set at the level of $400-500 \mathrm{GeV}$, and the limit on $\Lambda_{L L}$ (electron compositness scale) can be set to 3-4 $\mathrm{TeV}$ (90\% c.l.).

The value of $\sin ^{2} \theta_{W}^{\text {eff }}=0.2371 \pm 0.0025 \pm 0.0027$ at a $Q^{2}$ of $0.027 \mathrm{GeV}^{2}$. The standard model prediction at this $Q^{2}$ value is $0.2386 \pm 0.0006$ [4]. To compare these results with the other experiments, we convert this value to the renormalized mixing angle defined by $\overline{M S}$ (modified minimal subtraction) renormalization scheme: $\sin ^{2} \theta_{W}\left(m_{Z}\right)_{\overline{M S}}=0.2296 \pm 0.0038$. Figure 3 gives this comparison.

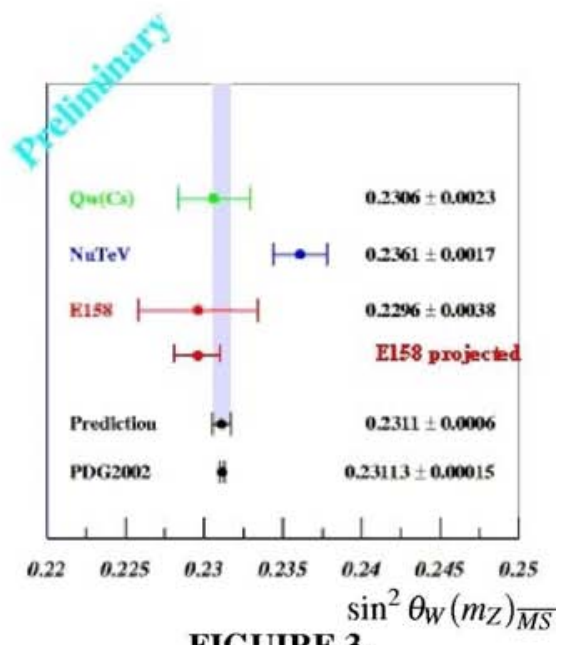

FIGUIRE 3.

\section{REFERENCES}

1. K. S. Kumar, et al., Mod. Phy. Lett. A, 10, 2979-2992(1995).

2. P. A. Souder, et al., SLAC-Proposal-E-158 (1997).

3. Derman, E., and Marciano, W. J., Annals of Physics, 121, 147-180 (1979).

4. Czarnecki, A., and Marciano, W. J., Physical Review D, 53, 1066-1072 (1996).

5. A combination of preliminary electroweak measurements and constraints on the standard model, Tech. Rep. CERN-EP/2001-98 (2001).

6. Czarnecki, A., and Marciano, W. J., Int. J. Mod. Phys. A, pp. 2365-2375 (2000). 


\section{Bibliography}

[1] T. D. Lee and C. N. Yang, Phys. Rev. 104 (1956) 254-258.

[2] C. S. Wu et al., Phys. Rev. 105 (1957) 1413-1414.

[3] C. Y. Prescott et al., Phys. Rev. Lett. B84 (1979) 524.

[4] P. A. Souder et al., SLAC-Proposal-E-158 (1997).

[5] K. Kumar et al., Mod. Phys. Lett. A, Vol. 10, No. 39 (1995) 2979-2992

[6] W. Marciano, AIP Conf. Proc. Vol. 542, Issue 1 (2000) 48-59.

[7] W. Marciano, Phys. Rev. D 60 (1999) 93-106.

[8] Precision Electroweak Measurements and "New Physics"., W. Marciano, BNL-HET$99 / 3$.

[9] R. S. vanDyck Jr., P. B. Schwinberg, and H. G. Dehmelt, Phys. Rev. Lett. 59 (1987) 26.

[10] W. Marciano, Phys. Rev. D 20 (1979) 274.

[11] M. Davier and A. Hocker, Phys. Lett. B 439 (1998) 427.

[12] Y. B. Zel'dovich, JEPT 9 (1959) 682.

[13] P. A. Souder et al., Phys. Rev. Lett. 65 (1990) 694. 
[14] E. Derman and W. Marciano, Ann. Phys. 121 (1979) 147.

[15] A. Czarnecki and W. Marciano, Phys. Rev. D 53 (1996) 1066.

[16] D. Relyea, Ph. D. Thesis, Princeton University (unpublished, 2003).

[17] M. L. Swartz, in Proc. of the 19th International Symposium on Lepton and Photon Interactions at High-Energy, Stanford, CA, (1999).

[18] P. C. Rowson, Dong Su, and Stephane Willocq, Ann. Rev. Nucl. Part. Sci. 51 (2001) $345-412$.

[19] A Combination of Preliminary Electroweak Measurements and Constraints on the Standard Model. Technical Report CERN-EP/2001-98. (2001).

[20] A. Czarnecki and W. Marciano, Int. J. Mod. Phys. A 13 (1998) 2235-2244.

[21] A. Czarnecki and W. Marciano, Int. J. Mod. Phys. A 15 (2000) 2365-2375.

[22] C. S. Wood et al., Can. J. Phys. 77 (1999) 7-75.

[23] K. S. McFarland et al., Eur. Phys. Jour. C31, (1998) 509.

[24] E. J. Eichten, K. D. Lane, and M. E. Peskin, Phys. Rev. Lett., 50 (1983) 811.

[25] B. Schrempp et al., Nucl. Phys. B 296, 1 (1988).

[26] B. W. Lynn, M. E. Peskin, and R. G. Stuart, Physics at LEP, CERN-86-02 (1986).

[27] M. E. Peskin and T. Takeuchi, Phys. Rev. D 46 (1992) 381.

[28] M. E. Peskin and T. Takeuchi, Phys. Rev. Lett 65 (1990) 964.

[29] D. C. Kennedy and B. W. Lunn, Nucl. Phys. B 322 (1989) 1.

[30] I. Maksymyk et al., Phys. Rev. D 50 (1994) 529. 
[31] B. Grinstein and M. B. Wise, Phys. Lett. B (1991) 265:326.

[32] J. L. Hewett et al., Indirect Probes of New Physics., 1996 SLAC-PUB-7088.

[33] A Study of the E158 Spectrometer and Backgrounds, R. Boyce et al., (unpublished) E158 documentation.

[34] A. E. Bonder and E. L. Saldin, Nucl. Inst. Meth. 195, (1982) 577.

[35] S. A. Belomesthnykh et al., Nucl. Inst. Meth. 227, (1984) 173.

[36] M. S. Griffo, (unpublished) E158 Technote 4 (2000).

[37] T. B. Humensky, Ph. D. Thesis, Princeton University (unpublished, 2003).

[38] T. B. Humensky et al. SLAC-PUB-9381. Submitted to Nucl. Inst. Meth. A.

[39] Paul Horowitz and Willard Hill, The Art of Electronics, Cambridge University Press, Cambridge, 1980 (pp. 437-442).

[40] T. Maruyana et al., Nucl. Inst. Meth. A, 492 (2002) 199-211.

[41] K. Unser, Proc. IEEE Particle Accelerator. Conf. (1989) 71.

[42] M. Cooke, (unpublished) E158 Technote 48 (2003).

[43] Z.D. Farkas et al., preprint SLAC-PUB-1823 (1976).

[44] D.H. Whittum, Yu.G. Kolomensky, Rev. Sci. Instrum. 70, (1999) 2300.

[45] B. Tonguc and M. Woods, (unpublished) E158 Technote 12 (2002).

[46] J. Gao et al., Nucl. Inst. and Meth. A 498 (2003) 90-100.

[47] D. Lhuillier, (unpublished) E158 Technote 42 (2003).

[48] E. Chudakov, Polarimetry: New Detector, (unpublished) E158 Documentation (2002). 
[49] P. Gorodetzky et al., Nucl. Instr. and Meth. A 361 (1995) 161.

[50] N. Akchurin et al., Nucl. Instr. and Meth. A 399 (1997) 202.

[51] P. A. Souder, Design of the Detector for E158., E158 documentation (unpublished, 1999).

[52] B. T. Tonguc, Ph. D. Thesis, Syracuse University (unpublished, 2003).

[53] D. Relyea, Moller Electronics Specifications, E158 TechNote 26 (2001).

[54] D. Relyea Run I Moller Asymmetry Analysis, E158 TechNote 35 (2003).

[55] P. Bosted, Radiative Corrections for E158, E158 TechNote 23 (2002).

[56] V. A. Zykunov, Electroweak radiative effects in Moller scattering of polarized particles, to be published in Sov. J. Nucl. Phys. 